Florida International University

FIU Digital Commons

3-16-2020

\title{
Visible Optical Coherence Tomography based Multimodal Imaging for Quantification of Retinal Lipofuscin
}

\author{
ZAHRA NAFAR \\ znafa001@fiu.edu
}

Follow this and additional works at: https://digitalcommons.fiu.edu/etd

Part of the Bioimaging and Biomedical Optics Commons, Biomedical Devices and Instrumentation Commons, and the Vision Science Commons

\section{Recommended Citation}

NAFAR, ZAHRA, "Visible Optical Coherence Tomography based Multimodal Imaging for Quantification of Retinal Lipofuscin" (2020). FIU Electronic Theses and Dissertations. 4413.

https://digitalcommons.fiu.edu/etd/4413

This work is brought to you for free and open access by the University Graduate School at FIU Digital Commons. It has been accepted for inclusion in FIU Electronic Theses and Dissertations by an authorized administrator of FIU Digital Commons. For more information, please contact dcc@fiu.edu. 


\title{
FLORIDA INTERNATIONAL UNIVERSITY
}

Miami, Florida

\section{VISIBLE OPTICAL COHERENCE TOMOGRAPHY BASED MULTIMODAL IMAGING FOR QUANTIFICATION OF RETINAL LIPOFUSCIN}

\author{
A dissertation submitted in partial fulfillment of \\ the requirements for the degree of \\ DOCTOR OF PHILOSOPHY \\ in
}

BIOMEDICAL ENGINEERING

by

Zahra Nafar

2020 
To: Dean John L. Volakis

College of Engineering and Computing

This dissertation, written by Zahra Nafar, and entitled Visible Optical Coherence Tomography based Multimodal Imaging for Quantification of Retinal Lipofuscin, having been approved in respect to style and intellectual content, is referred to you for judgment.

We have read this dissertation and recommend that it be approved.

$\begin{array}{r}\text { Rong Wen } \\ \hline \text { Jessica Ramella-Roman } \\ \hline \text { Richard Bone } \\ \hline \text { Wei-Chiang Lin } \\ \hline \text { Shuliang Jiao, Major Professor }\end{array}$

Date of Defense: March 16, 2020

The dissertation of Zahra Nafar is approved.

Dean John L.Volakis College of Engineering and Computing

Andrés G. Gil Vice President for Research and Economic Development and Dean of the University Graduate School

Florida International University, 2020 
C Copyright 2020 by Zahra Nafar

All rights reserved.

Authorship Attribution Statement:

This dissertation contains material from 4 published papers in the following peerreviewed journals.

The content of chapter 3 is published in Biomedical Optics Express as Zahra Nafar, Minshan Jiang, Rong Wen, and Shuliang Jiao," Visible-light optical coherence tomography-based multimodal retinal imaging for improvement of fluorescent intensity quantification".

The content of chapter 4 is published in Biomedical Optics Express as Zahra Nafar, Rong Wen, and Shuliang Jiao," Visible light OCT-based quantitative imaging of lipofuscin in the retinal pigment epithelium with standard reference targets".

The content of chapter 7 is published in Scientific Reports - Nature journal as Zahra Nafar, Rong Wen, Ziqiang Guan, Yiwen Li and Shuliang Jiao,” Quantifying lipofuscin in retinal pigment epithelium in vivo by visible-light optical coherence tomography-based multimodal imaging”.

In addition, the content of this dissertation is partially reviewed and published in Experimental Biology and Medicine journal as Zahra Nafar, Rong Wen, and Shuliang Jiao, "Visible-light optical coherence tomography-based multimodal system for quantitative fundus autofluorescence imaging”. 


\section{DEDICATION}

I dedicate this dissertation to my mother, Aghdas Nesarirad Moghaddam, who has always been and will be my inspiration in life. 


\section{ACKNOWLEDGMENTS}

I would like to thank my major advisor Professor Shuliang Jiao for guiding me throughout this journey and my dissertation committee members, Dr. Jessica Ramella-Roman, Prof. Rong Wen, Dr. Wei-Chiang Lin, and Prof. Richard Bone, for their continuous support. I would like to extend my gratitude to Dr. my lab members Dr. Tan Liu for training me at the beginning of this journey, Dr. Rui Zhou for helping me with software development, Dr. Shanhui Fan for helping me with human retinal imaging, Nusrat Yeasmin and Khaleel Atkinson for helping me with spectroscopic measurements. I would also like to acknowledge the assistance provided by Dr. Rong Wen and Dr. Yiwen Li from the Bascom Palmer Eye Institute for the synthesis of A2E, and Dr. Ziqiang Guan from Duke University for performing the mass spectroscopic measurements.

I am grateful to my parents, Aghdas Nesarirad Moghaddam and Saeid Nafar, my sweetest sister, Sara Nafar, and finally my husband Dr. Reza Sheykhi for their unconditional love and support during these years. Also, I would like to express my gratitude to all my colleagues, and friends who have extensively helped me during my Ph.D., especially the science-loving people who volunteered to participate in this study.

I would like to acknowledge the financial support received throughout my Ph.D. study, including the Teaching Assistantship from the Department of Biomedical Engineering, Research Assistantship from the National Institute of Health grants, and Dissertation Year Fellowship from University Graduate School at Florida International University. 


\title{
ABSTRACT OF THE DISSERTATION \\ VISIBLE OPTICAL COHERENCE TOMOGRAPHY BASED MULTIMODAL IMAGING FOR QUANTIFICATION OF RETINAL LIPOFUSCIN
}

\author{
by \\ Zahra Nafar \\ Florida International University, 2020 \\ Miami, Florida \\ Professor Shuliang Jiao, Major Professor
}

Retinal degeneration is the leading cause of irreversible low vision and blindness in the world, that describes conditions characterized by progressive loss of photoreceptors. Retinal Pigment Epithelium (RPE) is located under photoreceptors' outer segments and plays an important role in the maintenance of photoreceptors by completing the visual cycle and phagocytosis of shed photoreceptor outer segments. Lipofuscin, a byproduct of the visual cycle, is a nondegradable compound that accumulates in the RPE cells and eventually damages the RPE cells and inevitably causes photoreceptor degeneration.

Lipofuscin is the major cause of fundus fluorescence that can be detected by Fundus Autofluorescent (FAF) imaging systems. Reliable and quantified FAF values are necessary for lipofuscin quantification which can be a significant tool in the diagnosis of retinal degenerative disease in early stages and provide a better opportunity for treatment before the loss of vision stage. However, FAF is attenuated by the ocular media prior to the RPE, including cornea, lens, vitreous body, retinal layers in front of the RPE, and the melanin granules within the RPE cells that migrate to the apical region upon light exposure. This attenuation varies among people and for an individual over time and cannot be measured 
directly, thus hurdles measurement of the true FAF values. Further, differences in acquisition systems such as illumination power and detector sensitivity, directly affect the measured FAF. This issue has been addressed by implementing a reference target in the FAF imaging system. Normalizing the FAF signal to that of the target eliminates the dependency on the acquisition parameters. However, the issue of pre-RPE and RPE melanin attenuation remains unresolved. Further, the fluorescence characteristics of the commercially available fluorescent reference are quite different than retinal lipofuscin that challenges the quantification of the absolute amount of lipofuscin in the RPE.

In this dissertation, we propose a new multimodal imaging system based on visible-light optical coherence tomography (VIS-OCT) that provides a three-dimensional image. The technology simultaneously acquires VIS-OCT and FAF with a single broadband visible light source. Since both images are originated from the same group of photons and travel through the same ocular media at the same time, the attenuation factor is similar in both modalities. Therefore, by normalizing FAF by VIS-OCT of the RPE layer, the attenuation of the pre_RPE media can be eliminated. Further, we implemented two reference targets to quantify VIS-OCT and FAF and eliminate the dependency on acquisition parameters. These references were later substituted by a single customized reference that consists of the major lipofuscin fluorophore, called A2E. The quantitative imaging independent of system fluctuation, and attenuation of pre-RPE and RPE melanin was successfully tested on retinal simulating phantoms, in vivo on the animal retina, and human subjects. The in vivo quantification in small animals linearly correlates with $\mathrm{A} 2 \mathrm{E}$ content measured by mass spectrometry. Quantitative imaging of human retinas is consistent with linear accumulation of lipofuscin with age. The VIS-OCT-FAF has the potential for clinical diagnosis. 


\section{TABLE OF CONTENTS}

CHAPTER

PAGE

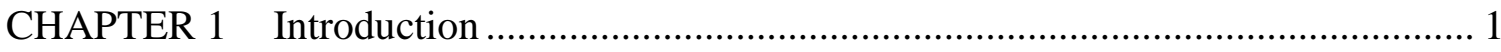

1.1 Significance of Lipofuscin Quantification .......................................................... 1

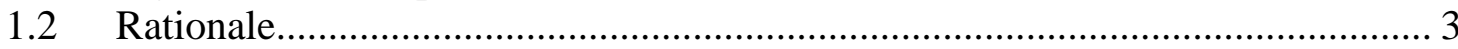

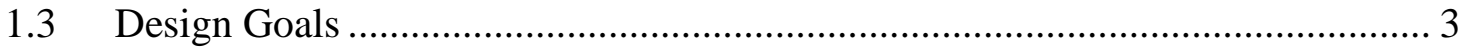

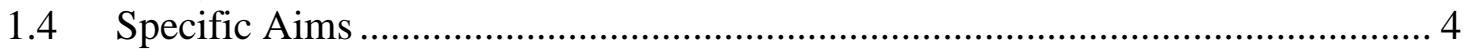

1.4.1 Specific Aim 1: VIS-OCT-FAF for pre-RPE attenuation compensation ..... 4

1.4.2 Specific Aim 2: VIS-OCT-FAF with reference target for quantification..... 4

1.4.3 Specific Aim 3: Quantification of RPE lipofuscin independent of RPE

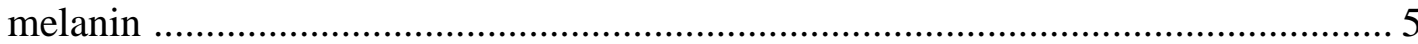

1.5 Organization of this Dissertation.............................................................. 5

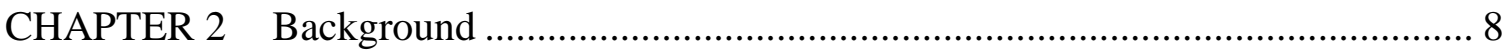

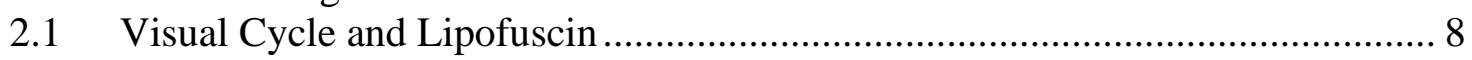

2.1.1 Retinal Pigment Epithelium .................................................................... 9

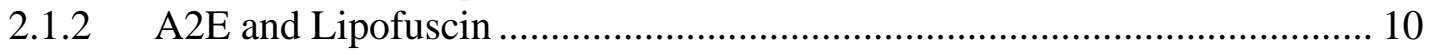

2.2 FAF Imaging System ………………………........................................... 12

2.2.1 qAF quantification with the reference target ............................................ 14

2.3 Visible Light Optical Coherence Tomography ………………….................... 15

2.3.1 System design and challenges.................................................................. 16

2.3.2 VIS-OCT and FAF.......................................................................... 17

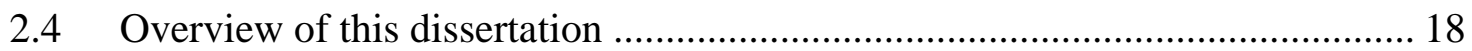

CHAPTER 3 Development of Simultaneous VIS-OCT and FAF for compensation

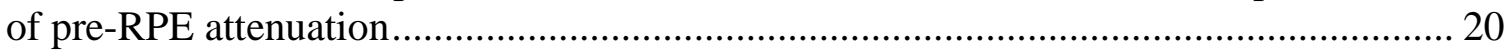

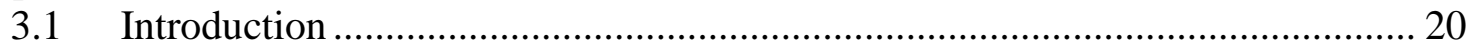

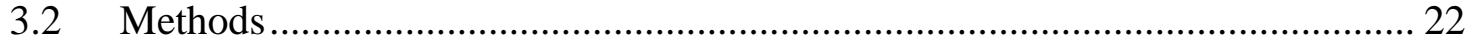

3.2.1 Imaging system ........................................................................... 22

3.2.2 Modeling of OCT and AF signals of the retina ........................................ 24

3.2.3 Animal experiments ........................................................................ 26

3.2.4 Phantom construction.......................................................................... 26

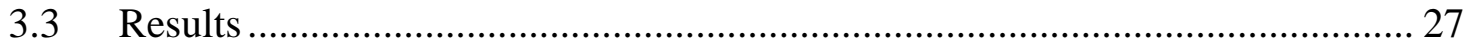

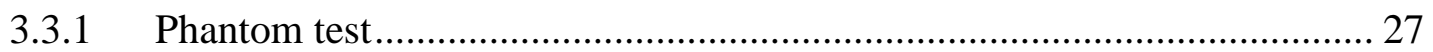

3.3.2 in vivo simultaneous VIS-OCT and retina AF imaging ............................ 28

3.3.3 in vivo simultaneous VIS-OCT and retinal FA ......................................... 31

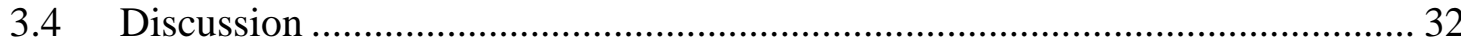

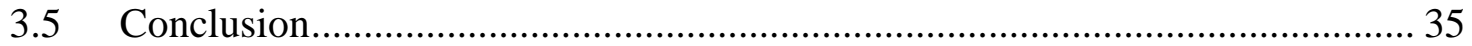

CHAPTER 4 Quantification of VIS-OCT and FAF for elimination of acquisition

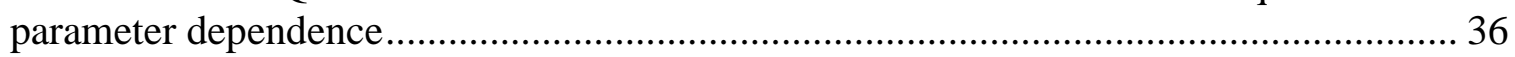

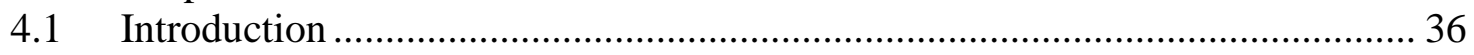

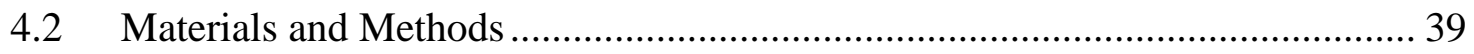

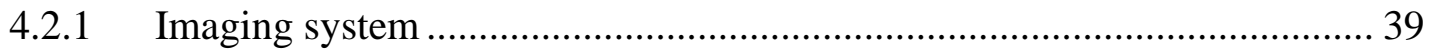


4.2.2 Mathematical model of OCT reflectance and AF signals......................... 42

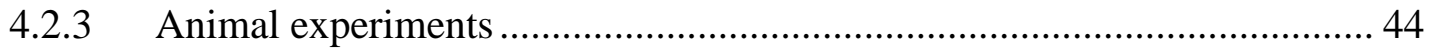

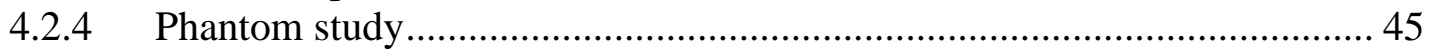

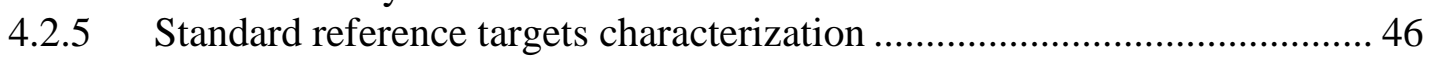

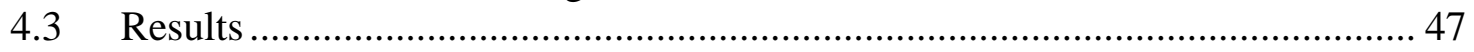

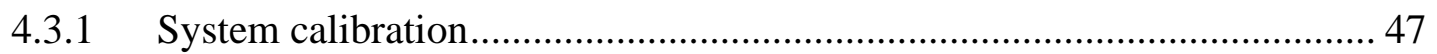

4.3.2 in vivo simultaneous VIS-OCT and retina FAF imaging ....................... 51

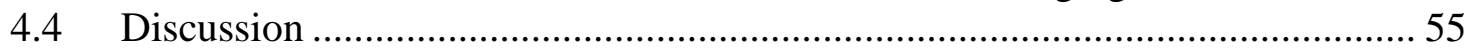

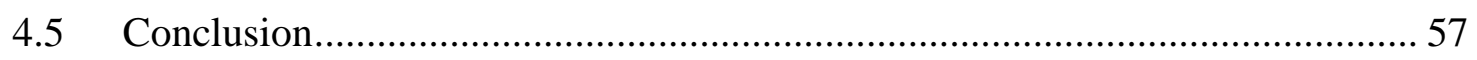

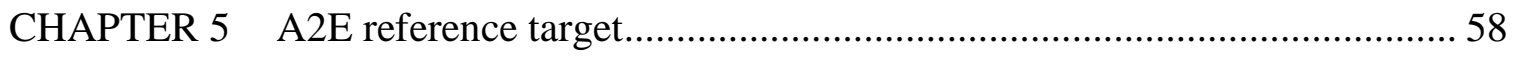

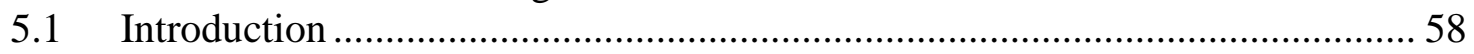

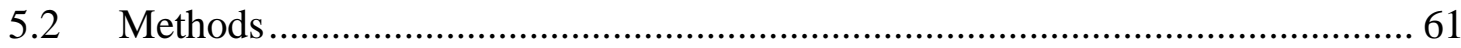

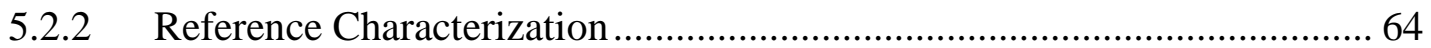

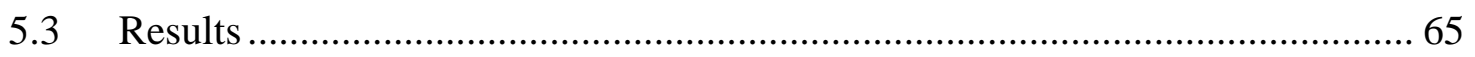

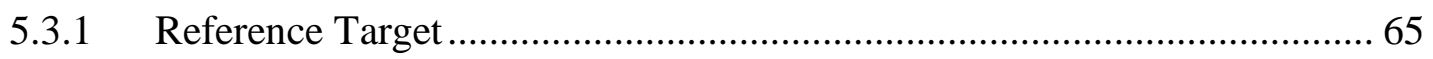

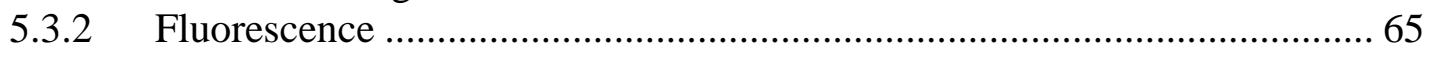

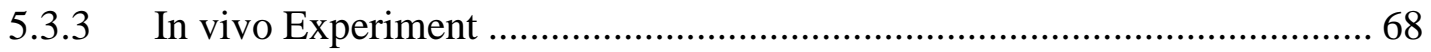

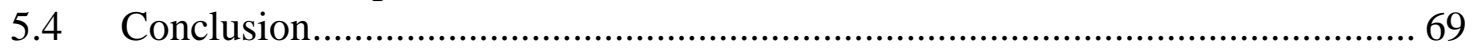

CHAPTER 6 RPE Simulating Phantoms for VIS-OCT-FAF .................................... 70

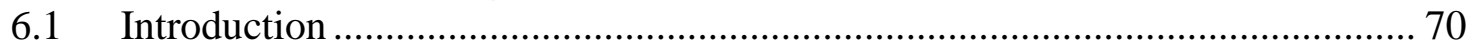

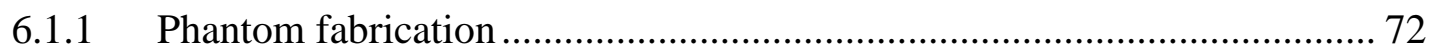

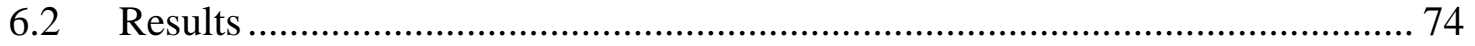

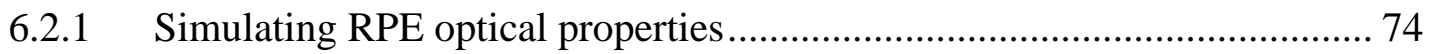

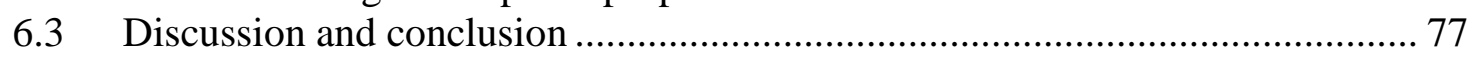

CHAPTER 7 Quantification of Lipofusicn/A2E concentration with A2E reference

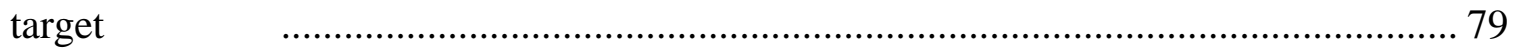

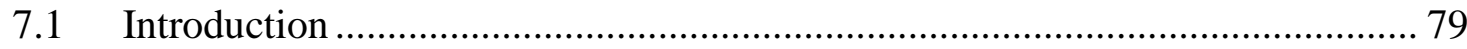

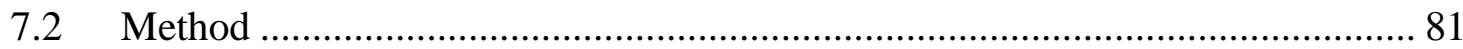

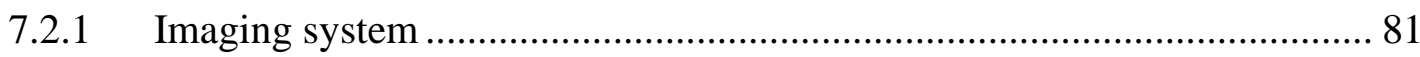

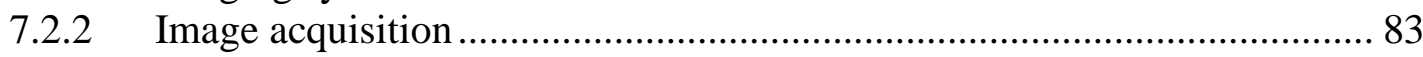

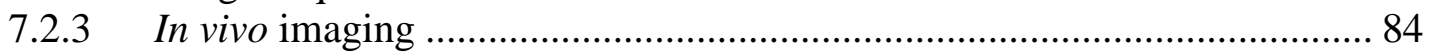

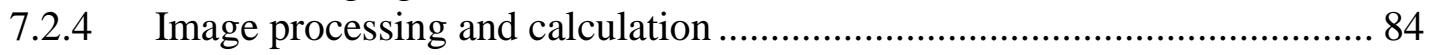

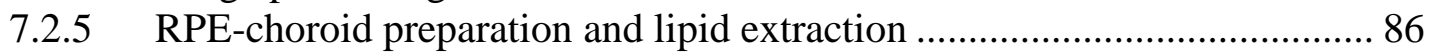

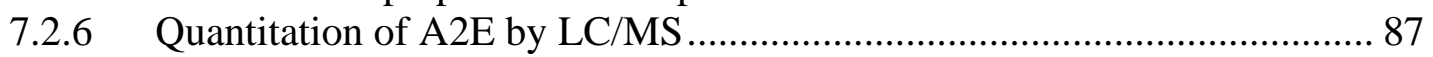

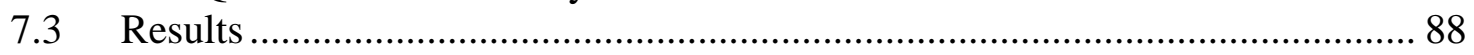

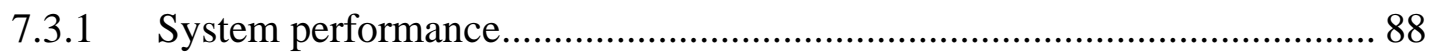

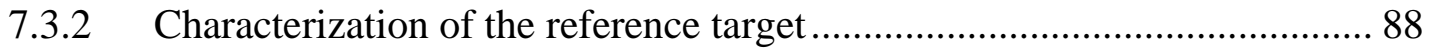

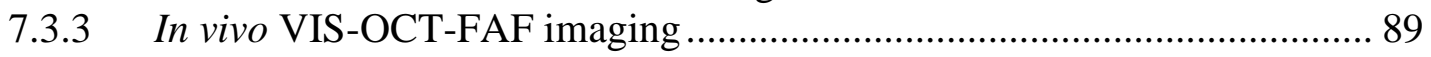

7.3.4 Compensation of signal attenuation by RPE melanin.............................. 90

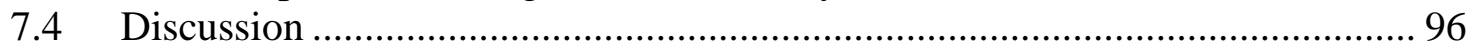

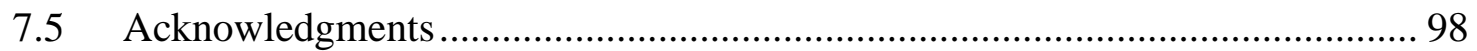

CHAPTER 8 Translation of VIS-OCT-FAF for clinical use .................................... 99 


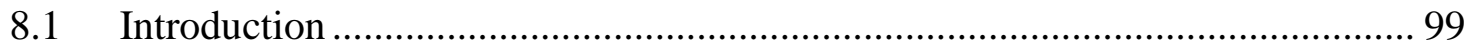

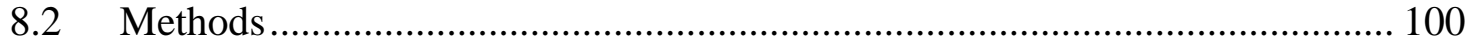

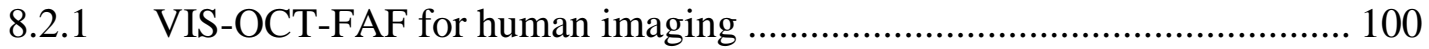

8.2.2 Light Safety Consideration ................................................................. 103

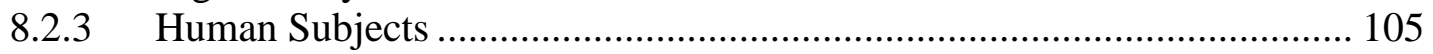

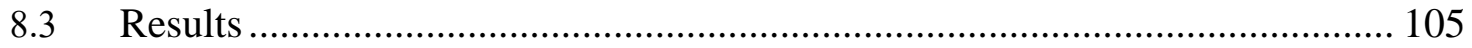

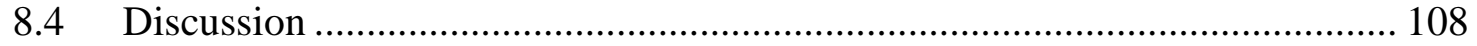

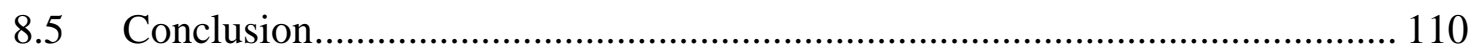

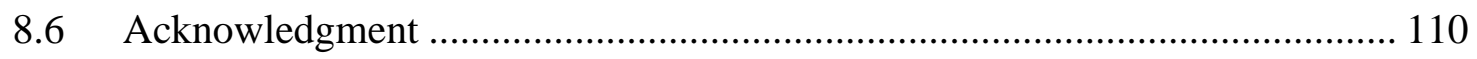

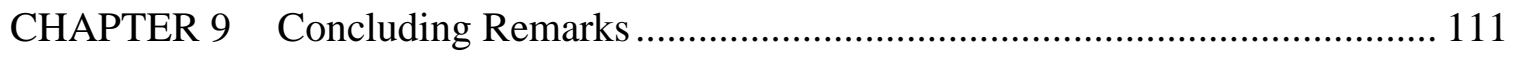

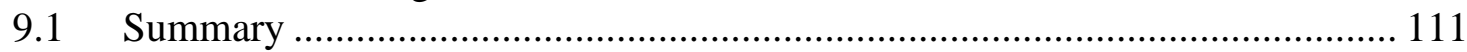

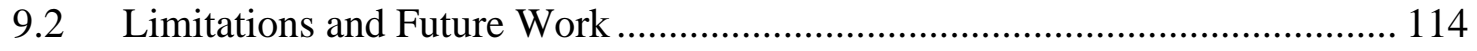

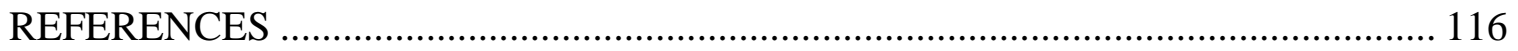

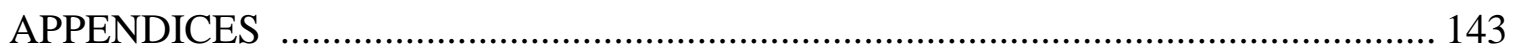

VITA 


\section{LIST OF FIGURES}

FIGURE

PAGE

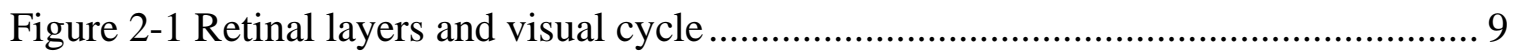

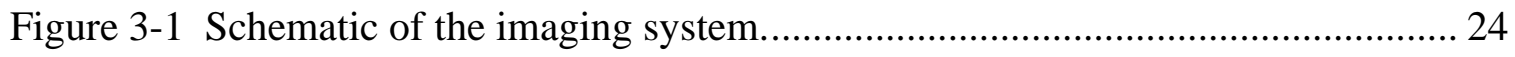

Figure 3-2 Phantom simulation experiment............................................................. 28

Figure 3-3 VIS-OCT and AF images simultaneously acquired in vivo........................ 29

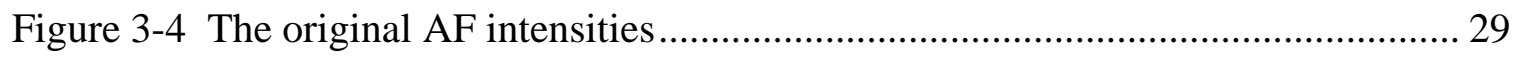

Figure 3-5 VIS-OCT and AF images simultaneously acquired in vivo........................ 31

Figure 4-1 Schematic of VIS-OCT-FAF with two references.................................. 41

Figure 4-2 Quantitative AF signals from the model eye......................................... 48

Figure 4-3 OCT reflectance signals from the model eye ......................................... 49

Figure 4-4 Absorption and fluorescence spectrums fluorescent slides......................... 50

Figure 4-5 Simultaneous FAF and OCT images of a 2-months-old albino rat................ 52

Figure 4-6 Simultaneous FAF and OCT images of a 14-months-old albino rat.............. 53

Figure 4-7 Comparison of the qAF, qOCT, and qAF/qOCT ..................................... 53

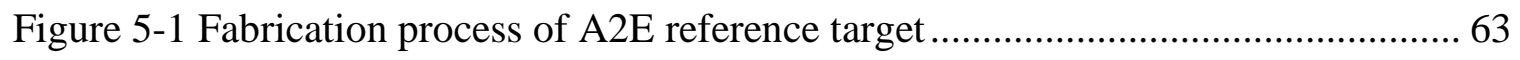

Figure 5-2 PMMA-A2E homogenity test and final A2E target.................................. 63

Figure 5-3 Characterization methods of A2E reference ........................................... 65

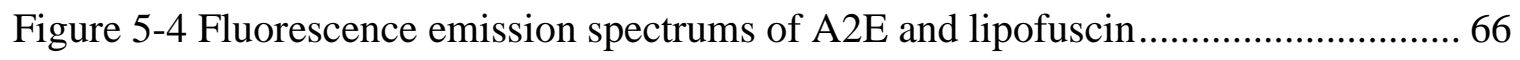

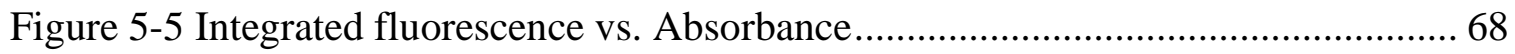

Figure 5-6 Simultaneous in vivo VIS-OCT and FAF of a 9-month-old pigmented rat.... 69

Figure 6-1 Evaluation of PDMS-TiO2 and PDMS-Carbon phantoms .......................... 75 
Figure 6-2 Empirical calculation of the optimum concentration of $\mathrm{TiO} 2$ and Carbon..... 76

Figure 6-3 Compensation of attenuation caused by RPE melanin in phantoms .............. 77

Figure 7-1 A schematic of the VIS-OCT-FAF imaging system with A2E reference....... 83

Figure 7-2 FAF and OCT images from albino (SD) and pigmented (LE) rats ................ 90

Figure 7-3 Image processing for data analysis ................................................... 91

Figure 7-4 Correlation between FAF intensities and A2E amounts ............................ 92

Figure 7-5 Correlation between FAF intensities and A2E amounts ........................... 94

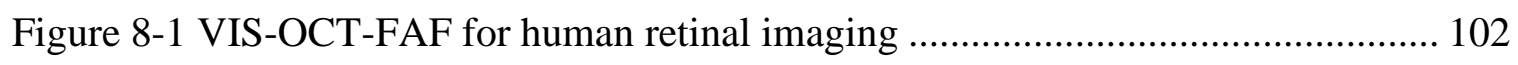

Figure 8-2 Simultaneous VIS-OCT and FAF of a 30-year-old volunteer .................... 106

Figure 8-3 Cross-sectional B-scan (2048 A-lines) of a 55-year-old volunteer............... 106

Figure 8-4 Comparison of qAF, qOCT, and qAF/qOCT of volunteers ....................... 107

Figure 8-5 Ethnicity-coded qAF, qOCT, and qAF/qOCT of volunteers ....................... 107 


\section{LIST OF ABBREVIATIONS}

Age-related macular Degeneration

AMD

American National Standards Institute

ANSI

area of interest

AOI

Autofluorescence

$\mathrm{AF}$

Barium Borate

BBO

Burch Membrane

$\mathrm{BM}$

Calibration factor

CF

compact laser diode

CLD

Confocal scanning laser ophthalmoscope

cSLO

curtain gas

CUR

de-clustering potential

DP

Deep Reactive Ion Etching

DRIE

Dichroic mirror

DM

dimethyl sulfoxide

DMSO

Fluorescent angiography

FA

focusing potential

FP

Fundus Autofluorescence

FAF

geographic atrophy

GA

High-Performance Liquid Chromatography

HPLC 
Inverse Adding and Doubling

IAD

ion source gas

GS

Ion spray voltage

IS

Liquid chromatography-mass spectrometry

LC/MS

Long Evans

LE

Maximum Permissible Exposure

MPE

molecular weight

MW

National Institutes of Health

NIH

Near-Infrared

NIR

Near-Infrared fundus autofluorescence

NIR-FAF

Near-Infrared light OCT

NIR-OCT

Neutral Density

ND

non-polarizing beam splitter

NBS

N-retinylidene-N-retinylethanolamine

A2E

$\mathrm{N}$-retinylidene-N-retinylphosphatidylethanolamine

A2PE

Optical Coherence Tomography

OCT

Optical Density

OD

Outer Segments

OS

phosphatidylcholine

PC

Phosphatidylethanolamine

PE

photoacoustic microscopy

PAM

Photomultiplier Tube

PMT 
$\begin{array}{ll}\text { Photoreceptor } & \text { PR }\end{array}$

Poly (methyl methacrylate) PMMA

$\begin{array}{ll}\text { Polydimethylsiloxane PDMS } & \text { PDS }\end{array}$

Quantified Autofluorescence $\quad$ qAF

$\begin{array}{ll}\text { Quantified OCT signal } & \text { qOCT }\end{array}$

Reference Autofluorescence RAF

Reference autofluorescent RAF

relative intensity noise $\quad$ RIN

Retinal Pigment Epithelium RPE

Scanning Electron Microscope SEM

Scanning Laser Ophthalmoscope SLO

Short wave fundus autofluorescence SW-FAF

Spectral-Domain Optical Coherence Tomography SD-OCT

Sprague Dawley $\quad$ SD

Standard deviation $\quad$ Std

Supercontinuum $\quad$ SC

$\begin{array}{ll}\text { Superluminescent diode } & \text { SLD }\end{array}$

time domain Optical Coherence Tomography TD-OCT

$\begin{array}{ll}\text { Titanium Oxide } & \mathrm{TiO} 2\end{array}$

$\begin{array}{lll}\text { Visible light OCT } & \text { VIS-OCT }\end{array}$

Visible OCT and FAF system $\quad$ VIS-OCT-FAF 


\section{CHAPTER 1 Introduction}

\subsection{Significance of Lipofuscin Quantification}

Millions of people worldwide suffer from retinal degenerative diseases with different degrees of vision loss ${ }^{1,2}$. Early diagnosis and treatment of diseases like Age-related Macular Degeneration (AMD) and Stargardt, is crucial in order to preserve vision and patients' quality of life. Some of the genetic and environmental factors such as low eye pigmentation, extreme light exposure, and smoking have been identified ${ }^{3}$. However, the underlying pathogenesis mechanism remains obscure ${ }^{4}$. Clinical studies suggest that the alterations in the RPE are the main causes of retinal degenerative disease. RPE is a monolayer of pigmented cells that are located under photoreceptors and is basically responsible for crucial maintenance of the photoreceptors by completing the visual cycle and daily phagocytosis of spent photoreceptor outer segments. Non-degradable byproducts of visual cycle build up in RPE cells in the form of granules called lipofuscin. One of the important changes in RPE is the accumulation of lipofuscin with age and more significantly in AMD. Lipofuscin granules are storage bodies of a mixture of lipids, proteins, and different fluorescent compounds, which are from incomplete digestion of phagocytosed photoreceptor outer segments ${ }^{5-7}$. A2E is a major fluorophore of lipofuscin that has been indicated in RPE atrophy ${ }^{8}$. Accumulation of lipofuscin/A2E and consecutively gradual changes of specific functions of RPE is one of the known causes of retinal degenerative diseases ${ }^{9-11}$. Lipofuscin/A2E is the major source of fundus autofluorescence (FAF) that can be excited between 480-510nm with an emission peak around $630 \mathrm{~nm}^{12}$. 
Hyperautofluorescent has shown correlations with the progression of diseases like AMD ${ }^{12,13}$, Stargardt ${ }^{14-16}$, and Best ${ }^{17,18}$. Thus, lipofuscin/A2E is a biomarker for blinding diseases like AMD and quantification of this compound through Fundus Autofluorescence (FAF) is important in diagnosis, progression monitoring and treatment evaluation of these diseases.

FAF imaging has been widely used in ophthalmology. It is commonly based on a Scanning Laser Ophthalmoscope (SLO) with 480nm wavelength for lipofuscin excitation. However, quantification of the FAF signal is challenged by differences in acquisition systems, more significantly opacity of ocular media in front of the RPE, and the presence of melanin granules in the RPE cells. The intensity of the FAF signal directly depends on the intensity of the excitation light reaching the fluorophores, and the sensitivity of the AF detector ${ }^{13}$. Further, the excitation light and emitted light are attenuated traveling through the ocular media including the cornea, lens, vitreous and aqueous humor, and pre-RPE retinal layers that are located in front of the RPE lipofuscin. Additionally, melanin granules in the RPE cells travel to the apical regions to protect photoreceptor outer segments by absorbing the scattered light. This phenomenon also attenuates the FAF signal. The attenuation caused by pre-RPE media and RPE melanin cannot be measured directly and varies among people ${ }^{14}$ and for individuals over time ${ }^{15}$. Therefore, compensating for these variables is a necessary step for the quantification of FAF and consequently lipofuscin/A2E. In 2011, Delori ${ }^{16}$ introduced a fluorescent reference target to address the excitation light and detector sensitivity variation between instruments and over time, however, the issue of attenuation caused by pre-RPE media and RPE melanin remains to be addressed. 


\subsection{Rationale}

We designed and developed a multimodal imaging technology that simultaneously acquires VIS-OCT and FAF with a single broadband visible light source (VIS-OCT-FAF). Because of the depth resolving capability of OCT, the reflectance OCT signal of the RPE layer can be segmented. Since both AF and RPE reflectance are originated from the same group of photons and traveled through the same media, they carry the same attenuation information. Thus, RPE reflectance can be used as an internal reference for each person at a certain time. By normalizing FAF by simultaneous VIS-OCT of the RPE, we can compensate for the attenuation of ocular media prior to the RPE and melanin granules within the RPE cells. Further by implementing two reference targets (a fluorescent reference introduced by Delori and a reflectance reference for OCT) we will able to compensate for the fluctuation of parameters such as illumination power and detection sensitivity in each acquisition system. With overcoming these obstacles, it would be possible to measure the true FAF values and thus use them to quantify the actual lipofuscin/A2E content of RPE. By fabricating a customized reference target with synthesized A2E, that has the same fluorescence characteristics of lipofuscin, quantification of absolute content of lipofuscin with an A2E-equivalent unit will become possible.

\subsection{Design Goals}

The overall goal of this dissertation is the development, characterization, and assessment of VIS-OCT-FAF for in vivo quantification of retinal lipofuscin or its major component A2E. The multimodal imaging technology should be able to: 
- Simultaneously acquire FAF and VIS-OCT with a single visible light source

- Compensate for the attenuation of ocular media in front of the RPE where lipofuscin is present

- Compensate for the changes in illumination power and detection sensitivity

- Compensate for the attenuation caused by melanin granules in the RPE

- Provide a linear correlation between in vivo measurements and actual A2E content of RPE

- Be suitable for in vivo quantitative imaging of human retina

The specific aims listed below serve to accomplish these goals and further translation of the VIS-OCT-FAF for clinical use.

\subsection{Specific Aims}

\subsubsection{Specific Aim 1: VIS-OCT-FAF for pre-RPE attenuation compensation}

a) To design and develop a VIS-OCT multimodal imaging system that simultaneously acquires VIS-OCT and FAF with a single broadband light source.

b) To assess the capability of the system to eliminate attenuation of the media in front of the fluorescent layer on phantoms.

c) To assess the imaging technology in vivo on small animals' retina.

\subsubsection{Specific Aim 2: VIS-OCT-FAF with reference target for quantification}

a) To implement two reference targets into the intermediate retinal imaging plane of VIS-OCT-FAF. 
b) To assess the capability of the methodology in compensation of the illumination and detection variations on phantoms.

c) To assess the quantification method in vivo on small animals.

d) To fabricate and characterize a single customized reference target for both FAF and VIS-OCT with lipofuscin major fluorophore, A2E.

\subsubsection{Specific Aim 3: Quantification of RPE lipofuscin independent of RPE melanin}

a) To assess VIS-OCT-FAF with A2E reference in compensation of attenuation caused by RPE melanin in RPE simulating phantoms.

b) To assess the VIS-OCT-FAF with the A2E reference target in compensating for RPE melanin in vivo on small animals.

c) To prepare the imaging technology for clinal use

\subsection{Organization of this Dissertation}

Chapter 1: Introduction

This chapter discusses the significance and the rationale behind this project and the organization of this dissertation.

Chapter 2: Background

In this chapter, we reviewed the undergoing physiology changes in the retinal degenerative disease and diagnostic imaging technics that are currently used in clinics. We reviewed VIS-OCT and FAF, the efforts that have been done for quantification of FAF, and the 
limitations of the available qAF. Then we introduce VIS-OCT-FAF to overcome the limitations caused by acquisition systems variations and attenuation by ocular media.

\section{Chapter 3: Simultaneous VIS-OCT and FAF}

This chapter reviews the design and development of the VIS-OCT-FAF system with a single broadband light source and assessment of its ability to compensate for the attenuation caused by the media in front of the RPE. The methodology was tested on a model eye and in vivo on rats' retina.

Chapter 4: VIS-OCT-FAF with two reference targets

This chapter reviews the implementation of two reference targets into the VIS-OCT-FAF imaging system for the quantification of both OCT and FAF signals. Then we assessed the independence of the measurements to the acquisition system parameters such as illumination power and detection sensitivity, and linear correlation of the results with fluorescent efficiency. The in vivo results suggested that the VIS-OCT-FAF method can compensate for attenuation caused by RPE melanin which was further investigated in the next chapter.

\section{Chapter 5: A2E reference Target}

In this chapter, we explain the fabrication process of the A2E reference target, the methods and the results of optical properties characterization.

Chapter 6: RPE simulating Phantoms 
This chapter focuses on the fabrication process of RPE simulating phantoms, and the measurement of the optical properties. Then we assess VIS-OCT-FAF in compensation of RPE melanin attenuation in RPE phantoms.

Chapter 7: in vivo quantification with VIS-OCT-FAF and A2E reference target

This chapter reviews the fabrication of a single customized reference target for both VISOCT and FAF, consisting of synthesized A2E which itself is the major fluorescent biomarker in FAF. Then we assessed the methodology in compensation of attenuation caused by RPE melanin and compared in vivo quantification of lipofuscin with mass spectrometry.

Chapter 6: Translation of VIS-OCT-FAF for clinical use

In this chapter, we reviewed the optimization of the VIS-OCT-FAF system for in vivo human retinal imaging. Then explains the assessment of quantitative imaging of lipofuscin in human retinal.

Chapter 7: Conclusion

Concluding remarks include a summary of the developed technology, its capabilities, and limitations. Further, the future direction toward the clinical usefulness of the technology has been discussed. 


\section{CHAPTER 2 Background}

\subsection{Visual Cycle and Lipofuscin}

Figure 2-1(a) illustrates the anatomy of the human eye: cornea, aqueous humor, lens, vitreous body, and several layers of the retina including nerve fiber layer, Ganglion cell layer, inner and outer plexiform layer, inner and outer nuclear layer, photoreceptor inner and outer segments, retinal pigment epithelium, Bruch membrane and choroid ${ }^{17}$. The visual process begins upon the absorption of a photon in the photoreceptor outer segments. Rod and cone photoreceptors exploit a light-sensitive derivative of vitamin A, 11-cisretinal, which is linked to an opsin protein capable of activating signaling pathways. In light, 11-cis retinal isomerizes to all-trans retinal that activates the opsin and initiates the phototransduction which itself activates the bipolar neurons to transmit the information to ganglion neurons whose axons exit the eye and carry the nerve impulses to the brain. ${ }^{18 \text {, }}$ ${ }^{19,20}$. Even though all-trans retinal is essential for the initiation of vision, it is not sensitive to light and new 11-cis retinal is necessary for the continuous function of photoreceptors. Therefore, all-trans retinal is converted back to 11-cis retinal through a series of chemical reactions called the visual cycle (Fig. 2-1(b)). ${ }^{21}$ 
(a)

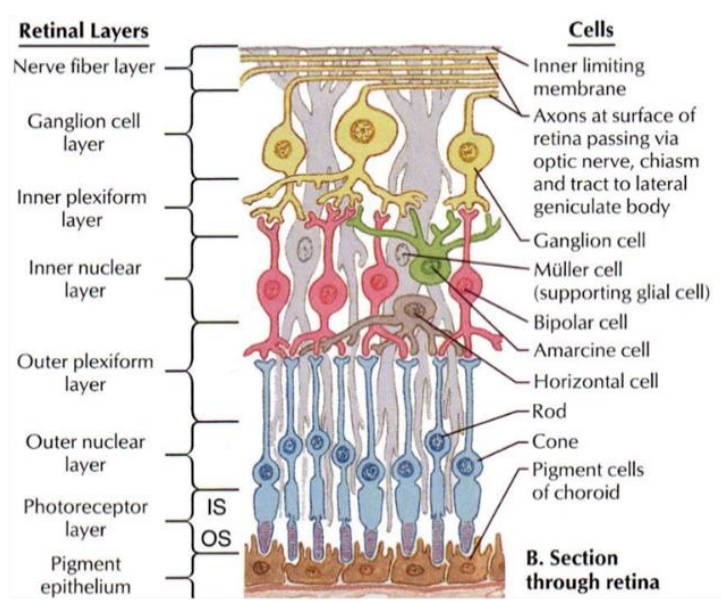

(b)

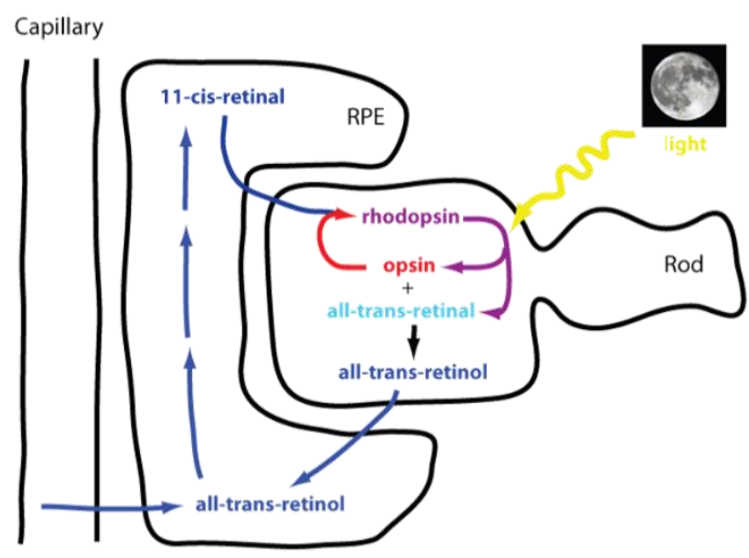

Figure 2-1 Retinal layers and visual cycle: a) Different layers of the retina and retinal cells ${ }^{17}$, b) Visual cycle in RPE and PRs. ${ }^{21}$

All-trans retinal is released from the activated opsin and bonds with phosphatidylethanolamine. This N-retinylidine-phosphatidylethanolamine is transported to the cytoplasm where it is reduced to all-trans-retinol. All-trans-retinol exists the photoreceptor and enters the RPE cell where it is converted to 11-cis retinal before it is transported back to the photoreceptor outer segment.

Therefore, the interactions between RPE and photoreceptors are essential for visual function. In fact, investigations on retinal degeneration have shown a strong dependence of RPE and photoreceptors to each other. For example, gene mutations expressed in RPE can lead to primary photoreceptor degeneration and gene mutations in photoreceptors can lead to primary RPE disease followed by loss of photoreceptors. ${ }^{1}$

\subsubsection{Retinal Pigment Epithelium}

RPE is located under the light-sensitive outer segments of photoreceptors. Its apical membrane faces the extracellular matrix enabling interaction between RPE and 
photoreceptor outer segments. And, its basolateral membrane is in contact with the Bruch's membrane that provides an interaction matrix for RPE with blood flow in the choriocapillaris. $^{2}$

Besides regeneration of 11-cis retinal, RPE is also responsible for phagocytosis of spent photoreceptors. In the human eye, the lens focuses the light on the macula that results in a strong concentration of photo-oxidative energy in the retina. On the other hand, the oxygen extraction from the choroid is relatively slow which causes the oxygen saturation of more than $90 \%$ in the venous blood from the choroid. ${ }^{3,4}$ Therefore, RPE is exposed to an overflow of oxygen from the choroid and strong photo-oxidative energy from the retinal side. This photo-oxidation damages the photoreceptor outer segment tips and necessitates a constant renewal process. RPE contributes to this renewal process by phagocytosis of the shed photoreceptor outer segments which itself adds a load of free radicals to the RPE photo-oxidative environment. To maintain the structural integrity of the retina, RPE has several defense lines ${ }^{1}$. First, an important defense line is melanosomes that move inside the cytoplasm to the apical processes under light conditions and absorb scattered photons. This pigmentation in the RPE cells is also thought to improve the visual quality by absorbing the scattered stray light.

\subsubsection{A2E and Lipofuscin}

As we mentioned, by absorption of a photon in rod and cone photoreceptors outer segments, all-trans retinal is produced. Although a major portion of all-trans retinal is reduced to all-trans retinol, a small portion of it reacts with phosphatidylethanolamine (PE), leading to the formation of N-retinylidene-N-retinylphosphatidylethanolamine (A2PE) ${ }^{22}$. 
More significantly, excess mount of 11-cis retinal in rod photoreceptors can also form A2PE. During the phagocytosis process in RPE cells, the phosphatidyl group of A2EP is cleaved and generates N-retinylidene-N-retinylethanolamine (A2E) ${ }^{22}$. A2E and other incompletely degraded membrane material build up and form a complex heterogeneous mixture of intracellular compounds including oxidized proteins, lipids, carbohydrates, metals, and other bioorganic molecules, named lipofuscin ${ }^{22,23}$. Lipofuscin in RPE accumulates with age and more significantly in retinal degenerative diseases like Agerelated Macular Degeneration (AMD).

Lipofuscin affects RPE cells in several ways including generation of free radicals and oxidants that lead to oxidative damage ${ }^{24}$, and lysis of cell membrane. Lipofuscin and its major component, A2E, is phototoxic in RPE cells and is known to reduce lysosomal stability and cell viability ${ }^{25,26}$. Therefore, its accumulation weakens the RPE cells and their line of defense. The reduced capacity to absorb light and to compensate for oxidative damages are thought of as important factors in the initiation of the chain of events that leads to photoreceptors loss in AMD.

Lipofuscin is highly fluorescent and can effectively be excited between 480-510nm with maximum emission between $600-640 \mathrm{~nm}$. A2E is a major hydrophobic fluorescent component of lipofuscin ${ }^{9}$, thus, a major fluorophore of fundus autofluorescent (FAF). Taking advantage of fluorescence of lipofuscin/A2E, fundus autofluorescent (FAF) has been widely used for diagnosis and monitoring retinal degenerations specifically in AMD. In fact, hyperautofluorescent has shown correlations with the accumulation of A2E/lipofuscin and progression of diseases like AMD ${ }^{12,13}$, Stargardt ${ }^{14-16}$, and Best ${ }^{17,18}$. 
FAF is a novel technology that visualizes lipofuscin/A2E distribution and FAF signal intensity corresponds to the accumulation/concentration of lipofuscin/A2E. Therefore, quantification of true values of FAF is necessary for quantification of A2E/lipofuscin and consecutively diagnosis of diseased eyes in early stages.

\subsection{FAF Imaging System}

Widespread use of fundus angiography revealed the fluorescence properties of the fundus without exogenous fluorophores, known as FAF. FAF is simple, efficient and noninvasive in nature with valuable clinical applications. Delori measured the excitation and emission spectra of retinal autofluorescence by using a spectrophotometer. The results showed that lipofuscin, a byproduct of the visual cycle, is the dominant source of FAF.

Fundus autofluorescent is about two orders of the fluorescein angiogram background. In order to detect autofluorescence of the fundus, high sensitivity cameras and averaging of several scans are required to record AF signals with acceptable SNR. By the introduction of confocal scanning ophthalmoscope (cSLO) in $1998^{27}$, autofluorescence of the retinal was extracted by scanning the retina with a low powered laser beam and these considerations were addressed ${ }^{28}$. With the development of scanning laser ophthalmoscope (SLO), point by point acquisition of the FAF became possible and confocal optics proved to be effective in suppressing the out of focus AF signal ${ }^{27,28}$ and correcting for refractive errors of the ocular media ${ }^{29}$. FAF intensity and pattern are greatly dependent on the wavelength, for example currently commercialized near-infrared- (NIR-) FAF uses 787nm for excitation and dominantly excites melanin, whereas, visible- or short wave- (VIS- or SW-) FAF utilizes $488 \mathrm{~nm}$ excitation light for visualization of lipofuscin ${ }^{6}$. An argon blue 
laser is commonly used for the SW-FAF light source and emitted light is detected using wideband barrier filters with short cut-off wavelengths of 500, 515, and 521 from different manufacturers. Photodiodes and photocathodes have been used to detect the fluorescence emission $^{29}$. Photomultiplier tubes (PMTs) consisting of the photocathode, dynodes, and anode, are uniquely well suited for applications requiring low noise and high sensitivity detection.

FAF signal is dependent on the power of the excitation light reaching the fluorophore and the gain or sensitivity of the detector measuring the emitted light. Measuring the true values of the FAF signal is challenged by several factors. First the excitation and emission light travel through ocular media prior to the RPE and lipofuscin which introduced a round trip attenuation factor to the signal. The optical properties of these media vary among people, moreover, the opacity of ocular media, especially ocular lens, changes for individuals over time ${ }^{30-32}$. Second, the initial intensity of the illumination light and the sensitivity of the detector and the spatial filters used for both excitation and emission, directly affect the measured FAF signal and can change from one system to the other and might fluctuate over time ${ }^{16}$. Therefore, comparison of images from different people or from individuals at different examination times is challenged which consequently weakens the clinical usefulness of FAF for early diagnosis of retinal degeneration.

In 2011, Delori proposed the implementation of an internal reference target to address the excitation light and detector sensitivity variation between instruments and over time. By normalizing the FAF signal to AF of the internal reference, the effects of acquisition 
systems alterations were compensated. However, the varying attenuation of the ocular media in front of the RPE remained to be addressed.

\subsection{1 $\mathrm{qAF}$ quantification with the reference target}

Delori's reference target was implemented into the FAF imaging system at the retinal intermediate imaging plane $^{16}$. The fluorescence reference target, with Texas red fluorescence spectrum, was optically cemented to neutral density filters to reduce the fluorescence efficiency of the internal reference to that of the retina. The reference target covered a small portion of the raster scanning area, resulting in imaging the internal reference along with the fundus. By normalizing the FAF by reference autofluorescence (RAF) signal, the alterations of the imaging system were compensated. The quantification method was excessively used on the evaluation of Stargardt, AMD and ABCA4 carriers ${ }^{33-}$ 38. However, the method introduced a scaling factor from the retina to the intermediate imaging plane that depends on the axial length of the eye. Further, the transmission of the ocular media in excitation and emission wavelengths is another factor in the calculation of the quantified $\mathrm{AF}(\mathrm{qAF})$. To address this factor the optical density of the ocular media was estimated based on age and with reference to a 20 -year-old subject. Further, the subject's retina was bleached for a period of 20 to 30 seconds to reduce the photopigment absorption. Although the method effectively eliminated the acquisition system fluctuation effects, the quantification is dependent on the measurement of the axial length of the eye and estimation of relative transmission based on age ${ }^{16}$ which is not inclusive of other individual differences such as racial factors ${ }^{26,39,40}$ that might contribute to the attenuation of FAF. 


\subsection{Visible Light Optical Coherence Tomography}

Since 1990 when the first Optical Coherence Tomography (OCT) system was reported, the technology has greatly advanced in clinical studies, specifically in retinal imaging. OCT is an imaging technique based on low coherence interferometry consisting of four arms: sour e arm, sample arm, reference arm, detection arm. In time-domain OCT (TD-OCT) the path length of the reference arm is varied in time within the coherence length of the light source to achieve the reflectivity profile of the sample. High-speed OCT imaging became possible by the invention of Spectral Domain OCT (SD-OCT) that the reference arm is stationary and instead a broadband source and a spectrometer are used to measure the interference pattern as a function of wavelength. ${ }^{42}$

OCT provides a three-dimensional structural image of the biological tissues with fine lateral and axial resolution. The penetration depth of OCT systems is in millimeter range (1-3mm) and sufficient for retinal imaging. Early OCT systems worked with only nearinfrared light and superluminescent diode (SLD) ${ }^{42,43}$. In 2002 for the first time, visible light OCT was achieved by employing a photonic crystal fiber in combination with a sub15fs Ti: sapphire laser (FEMTOLASERS) with spectrum range between 535nm and 700nm

44. The lateral and axial resolution of OCT is proportional to the central wavelength of the light source, therefore, the VIS-OCT system offered a significantly better resolution. Further, a smaller bandwidth is needed to achieve a comparable resolution to NIR-OCT. Optical properties of the eye and retinal function and interaction with visible light, suggests using the visible light spectrum for better understanding the visual cycle and retinal pathology. In addition, due to the high scattering coefficients of biological tissues in the 
visible range, VIS-OCT provides a higher contrast between biological tissues compared to NIR-OCT. However, the imaging depth is sacrificed in VIS-OCT due to the higher optical attenuation of the tissue in shorter wavelengths ${ }^{45}$. In the case of retinal imaging, when deep penetration is not necessary, lower probing power can achieve similar imaging contrast as NIR-OCT. Further, shorter wavelengths are more sensitive to the absorption properties of the biological tissues, thus, VIS-OCT can utilize quantitative absorption measurements such as blood vessel oximetry ${ }^{46-50}$.

\subsubsection{System design and challenges}

A broadband light source is required for low coherence interferometry of OCT. OCT systems use different types of light sources with low temporal coherence, such as superluminescent diode (SLD), ultrafast lasers, and supercontinuum (SC), and swept sources. SLDs are relatively inexpensive and greatly used in commercial OCT systems. However, the central wavelength of SLDs is limited to 670-1600nm and not suitable for VIS-OCT ${ }^{46}$. Previously investigators used barium borate (BBO) to double the frequency of Ti: sapphire NIR lasers for VIS-OCT, however, the bandwidth of this light source in this method is limited ${ }^{49}$. Swept sources quickly sweep through a series of wavelengths instead of illuminating the sample with a broad spectrum at the same time. Therefore, the spectrometer can be replaced by a single element photodetector so the A-line rate and effective penetration depth will increase. Nevertheless, most of the swept-source lasers work in the NIR range and no reliable visible swept source is available. The most famous light source in recent VIS-OCT systems is SC sources with broad-spectrum from 400nm to $2500 \mathrm{~nm}$. Although SC has much higher relative intensity noise (RIN) compared to SLD, 
RIN can be decreased by increasing the pulse reputation rate ${ }^{51}$. Due to the excess noise from SC sources, longer integration time might be necessary which will result in a lower A-line rate and thus higher motion artifact. Also, retinal imaging sensitivity of the eye to visible light makes it even harder to maintain the visual comfort of the human subjects.

VIS-OCT has adopted both free space and fiber-based Michelson interferometers. While free space avoids excessive loss and imbalanced dispersion in the fiber couplers, fiberbased takes advantage of compactness, easy alignment, and easy maintenance. Due to the lower safety threshold of visible spectral range, the unbalanced splitting ratio is usually used in both free space and fiber-based VIS-OCT to maximize the detection of the backscattered signal from the tissue. In shorter wavelengths, the variation of the refractive indices is larger and consecutively optical lenses introduce a larger chromatic aberration in the visible range. To address this issue, Lichtenegger et al ${ }^{52}$, employed reflective collimators in spectrometer design to maintain an optimum focus across a wide spectrum. Also, the reflectivity of aluminum and gold-coated used mirrors is lower in the visible range and is preferable to substitute them with silver-coated or broadband dielectric mirrors.

\subsubsection{VIS-OCT and FAF}

Although VIS-OCT and FAF imaging provide essential information about the structure and function of the retina, respectively, these two modalities information are complementary and should be combined for accurate diagnosis and monitoring of the disease progression and treatment. Several studies on diagnosis and monitoring of the

treatment progress ${ }^{53}$ have adopted both OCT and FAF imaging for categorization of 
various stages of the disease ${ }^{54}$ and a better understanding of the alteration in retinal layers such as RPE, BM, and photoreceptors ${ }^{55}$. In 2006 FDA cleared simultaneous OCT and FAF and angiography that was developed by Heidelberg. In this setup, NIR superluminescent diode laser has been used for OCT images and an optically pumped solid-state lasergenerated $488 \mathrm{~nm}$ for excitation of FAF ${ }^{56}$. In vivo studies using Heidelberg, Spectralis HRA+OCT, have shown a correlation between FAF and OCT images, thus, providing a better understanding of pathological and morphological changes in the retina ${ }^{57-59}$.

In 2012 for the first time, simultaneous VIS-OCT and FAF imaging system, with a single light source centered at $415 \mathrm{~nm}$ was introduced ${ }^{60}$. To achieve the visible light source, light from a broadband Ti: Sapphire laser centered at $800 \mathrm{~nm}$ with $120 \mathrm{~nm}$ bandwidth was focused on a frequency doubling crystal (BBO). Then visible light with $8 \mathrm{~nm}$ bandwidth was exited from the BBO. Since both images are produced simultaneously by the same group of photons, the images are intrinsically registered, and no post-processing for image registration is needed in this method. However, the application of the technology was limited to the biomolecules that can be excited at $415 \mathrm{~nm}$. Further, due to the limited bandwidth of the frequency doubling method, the axial resolution of VIS-OCT was not satisfactory for retinal studies.

\subsection{Overview of this dissertation}

In this dissertation, we take advantage of the previous investigations and develop a new multimodal technology suitable for quantitative imaging of lipofuscin. The technology simultaneously acquires FAF and VIS-OCT with a single broadband visible light source 
centered at 480nm, utilizing an SC laser. Spectral-domain VIS-OCT uses a fiber-based Michelson interferometer and a spectrometer. Confocal detection of FAF is achieved with a cSLO setup and a PMT. We fabricated and implemented an internal reference target consisting of lipofuscin major fluorophore, A2E, into the retinal imaging plane of the imaging system. A NIR-OCT is coupled into the VIS-OCT-FAF system for alignment of the retina prior to image acquisition to minimize photo-bleaching of the fluorophore of interest. We will explain, design, development, and assessment of VIS-OCT-FAF in the measurement of true values of AF signals which are independent of transmission of ocular media and fluctuation of imaging systems. We will then illustrate in vivo quantification of RPE lipofuscin/A2E based on the VIS-OCT-FAF measurement technique with reference to the $\mathrm{A} 2 \mathrm{E}$ reference target. 


\section{CHAPTER 3 Development of Simultaneous VIS-OCT and FAF for}

compensation of pre-RPE attenuation

\subsection{Introduction}

Optical coherence tomography (OCT) ${ }^{61,62}$, retinal autofluorescence (AF) imaging ${ }^{63,64}$, and fluorescein angiography (FA) ${ }^{65,66}$ are three important imaging modalities in both ophthalmic clinics and research, which image different aspects of the retina. OCT is a lowcoherence interferometry-based imaging modality, which can provide mainly structural imaging of the retina with microscopic depth resolution. Retinal AF imaging maps the distribution of lipofuscin, the major source of AF in the retinal pigment epithelium (RPE). Lipofuscin is a complex lipid/protein aggregates formed in the RPE as nondegradable end products from phagocytosis of shed photoreceptor outer segments ${ }^{67}$, which accumulate with age. Lipofuscin has influences on the RPE cells in many ways that are believed to contribute to the pathogenesis of age-related macular degeneration (AMD) ${ }^{68,69}$. FA images fluorescence from exogenous contrast molecules administrated into the bloodstream to capture the blood circulations and identify vascular leakage in the retina. Because of the complementary nature of their contrast mechanisms, these imaging modalities are suitable candidates for being integrated into a multimodal imaging system. A broadband visible light source can be used to build such a multimodal imaging system since retinal AF and FA needs visible light for fluorophore excitation. Visible light OCT (VIS-OCT) is gaining 
more attention in molecular contrast imaging recently because many molecules of interest in the retina have absorption spectra in the visible light range $44,49,51,60,70$.

We recently achieved simultaneous OCT and AF imaging with a single broadband visible light source and successfully applied the imaging technique to monitor the accumulation of lipofuscin in the RPE cells in albino rats $44,49,51,60,70$. The current system was designed to compensate the signal attenuation by the media anterior to the RPE to tackle a technical difficulty in the quantification of absolute AF intensities. Quantification of the absolute AF intensities is a major issue that the current retinal $\mathrm{AF}$ imaging technologies are not able to address ${ }^{16}$. During AF imaging, the excitation light must travel through the media anterior to the RPE before exciting the fluorophores, and the AF signals generated in the RPE must travel through the same media before being detected by the system. Signals are attenuated by these tissues, including the cornea, the aqueous humor, the vitreous, and especially by the lens ${ }^{71}$.

The AF intensities changes in aging, as well as in certain pathological conditions, such as in AMD and Stargardt diseases. Monitoring AF changes would be informative for disease progression, for which quantitative accuracy is important to see the difference between individuals as well as longitudinal changes in the same eye. However, the measured AF in an image can be affected by many factors, including the optical properties of the anterior segments of the eye, which are different among different individuals and change over time; the intensity of the excitation light; and even alignment of the eye with the excitation light beam. Eliminating the influence of all the factors that are not related to the optical properties of the RPE would improve the quantification accuracy of AF imaging, which 
will make it possible to compare the overall AF intensities as well as changes in the spatial distribution of AF.

The current multimodal imaging system can simultaneously acquire OCT and AF imaging with a single broadband visible light source. Because the OCT signals are attenuated by the same media as the AF signals, the OCT signals can be used as an internal reference to compensate for the signal attenuation by the media anterior to the RPE. Thus, our VISOCT based multimodal imaging technique offers a unique opportunity for compensation of the anterior segment attenuation effects on retinal AF imaging. This technique can also be used for retinal FA imaging. This paper reports our recent progress in simultaneous VISOCT, retinal AF, and FA imaging.

\subsection{Methods}

\subsubsection{Imaging system}

A schematic of the experimental system is shown in Fig. 3-1. The system has two SDOCTs: one works in the NIR band (Fig. 3-1. red lines) and the other in the VIS band (Fig. 3-1. blue lines). The VIS-OCT uses a supercontinuum laser (SC) source (EXB-6, SuperK EXTREME, NKT Photonics, Denmark) equipped with a variable band-pass filter (SuperK Varia, NKT Photonics). The filtered output light (center wavelength: $480 \mathrm{~nm}$, bandwidth: $20 \mathrm{~nm}, 80 \mathrm{MHz}$ pulse rate) is delivered through a fiber delivery module. The VIS light is coupled into the source arm of a single-mode optical fiber-based Michelson interferometer. The NIR-OCT uses a superluminescent diode (SLD-37-HP, center wavelength: $840 \mathrm{~nm}$, bandwidth: $50 \mathrm{~nm}$, Superlum, Russia). After passing through an optical fiber isolator, the NIR light is coupled into another single-mode fiber-based Michaelson interferometer. The 
visible and NIR light beams, after exiting their corresponding optical fibers in the sample arms, are collimated and combined by using two dichroic mirrors (DM1: NT43-955, Edmund Optics, and DM2: DMLP505, Thorlabs). The combined light beam is scanned by an $\mathrm{x}-\mathrm{y}$ galvanometer $(6215 \mathrm{H}$, Cambridge $)$ scanner, and delivered into the eye by the combination of a relay lens (L1, $f=75 \mathrm{~mm}$, achromatic) and an ocular lens (L2, $f=20 \mathrm{~mm}$, achromatic). The VIS light power was $500 \mu \mathrm{W}$ before entering the eye, while the NIR light at the same location was $600 \mu \mathrm{W}$.

In the detection arm of each OCT subsystem, the reflected light from the sample and reference arms is collimated and detected by a spectrometer (SPEC1-2). The VIS-OCT spectrometer has an 1800 lines/mm transmission grating, a multi-element imaging lens (f $=150 \mathrm{~mm}$ ), and a line scan CCD camera (Aviiva-SM2-CL-2010, 2048 pixels with $10 \mu \mathrm{m}$ pixel size operating in 12-bit mode, e2V). The NIR-OCT uses a spectrometer with the same parameters as described in our previous publications ${ }^{72}$.

The system for AF detection is similar to that described in our previous publications ${ }^{70}$. In short, the back-traveling fluorescent photons emitted from the fluorophore pass through the two dichroic mirrors and a long-pass filter (FGL515M, cut-on wavelength: $515 \mathrm{~nm}$, Thorlabs), and are then focused into a $25 \mu \mathrm{m}$ pinhole by an achromatic doublet with a focal length of $30 \mathrm{~mm}$ (L3). The AF photons are detected by a PMT module (PMM02, Thorlabs). The outputs of the PMT are digitized by a multifunction data acquisition board (DAQ, PCIe-6361, National Instruments) at a sampling rate of 2M/s. At each scanning position on the retina, a total of 80 points corresponding to a sampling length of $30 \mu$ s were acquired. The amplitudes of the AF signal of these 80 points were averaged to form a single pixel of 
the AF image. Synchronization of the AF data acquisition, scanning of the galvanometer scanner and the OCT image acquisition was controlled by the multifunction DAQ board.

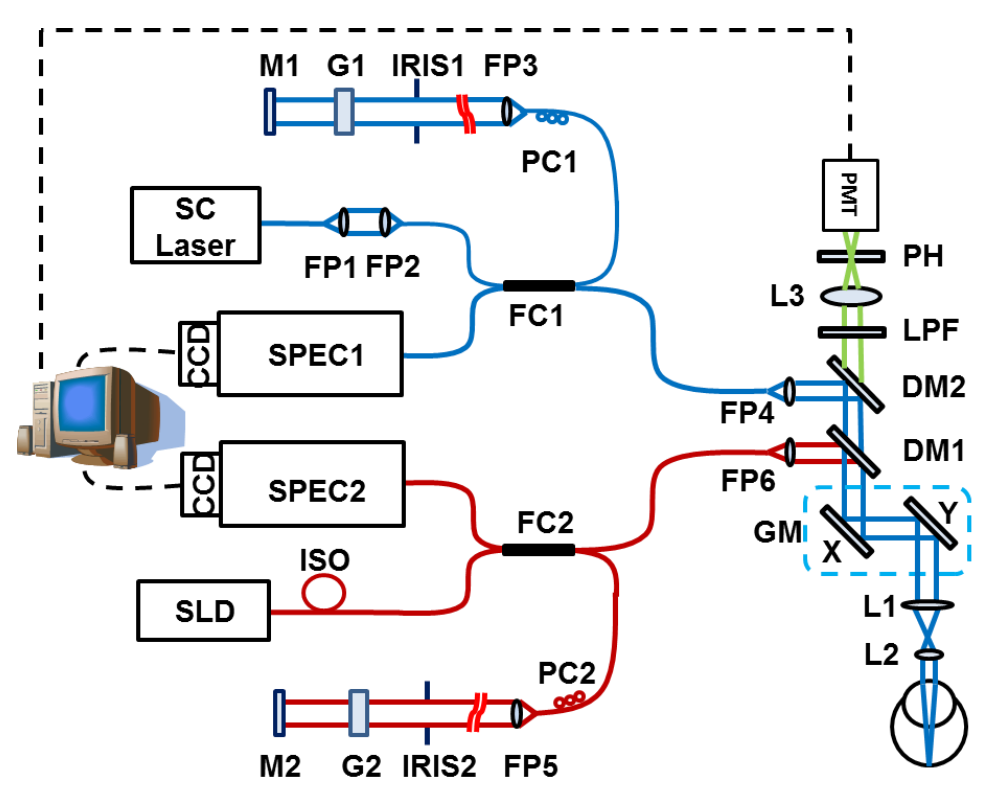

Figure 3-1 Schematic of the imaging system. SC: supercontinuum; SLD: superluminescent diode; DM1-2: dichroic mirror; L1-3: Achromatic lens; LPF: long-pass filter; PH: pinhole; FP1-7: Fiber collimating port; ISO: Isolator; FC1-2: 3dB fiber coupler; PC1-2: Polarization controller; G1-2: BK-7 glass plate; M1-2: Mirror; IRIS1-2: Iris.

\subsubsection{Modeling of OCT and AF signals of the retina}

The light propagations in the retina were studied by many research groups. With reference to the model of fundus reflection proposed by van de Kraats et al ${ }^{73,74}$, the measured light reflected from the retina can be divided into reflections from three sources, or three distinct layers of the eye. The first layer is the media anterior to the RPE. The second one is the RPE layer, where reflection by the RPE layer and absorption by the lipofuscin granules occurs. The third is the post-RPE layer, including the choroid and sclera. The intensity of the reflected light from pre- and the RPE layer can be expressed as: 


$$
\begin{gathered}
I_{\text {pre }}=I_{0} \cdot \tau_{\text {pre }} \lambda^{2} \cdot R_{\text {pre }}(\lambda) \\
I_{R P E}=I_{0} \cdot \tau_{\text {pre }}(\lambda)^{2} \cdot\left[1-R_{\text {pre }}\right]^{2} \cdot \tau_{R P E}(\lambda)^{2} \cdot R_{R P E}(\lambda)
\end{gathered}
$$

where $I_{0}$ is the light intensity incident into the eye, $I_{\text {pre }}$ and $I_{R P E}$ are the light intensities reflected from the pre-RPE and RPE layer, respectively; $\tau_{p r e}$ and $\tau_{R P E}$ are the transmittance of the pre-RPE and RPE layer, respectively; $R_{p r e}$ and $R_{R P E}$ are the reflectance of the preRPE and RPE layer, respectively.

The lipofuscin fluorescence intensity $\left(I_{A F-R P E}\right)$ detected can be expressed as:

$$
I_{A F-R P E}=I_{0} \cdot \tau_{\text {pre }}(\lambda)^{2} \cdot\left[1-R_{\text {pre }}\right]^{2} \cdot \varepsilon \cdot C \cdot d \cdot Q \cdot A_{d}
$$

where $\varepsilon$ is the extinction coefficient of the fluorescent pigment (lipofuscin), $\mathrm{C}$ is the lipofuscin concentration, $d$ is the RPE layer thickness, $\mathrm{Q}$ is the quantum yields of the fluorescence, $A_{d}$ is the detecting efficiency including the detection solid angle, the response of the PMT, the gain of the circuitry, and the digitization coefficient.

Assuming that $\tau_{\text {pre }}$ and $R_{\text {pre }}$ are the same for excitation light and the fluorescent, the ratio of $\mathrm{AF}$ and OCT signal is

$$
\frac{I_{A F}}{I_{R P E}}=\frac{\varepsilon \cdot C \cdot d \cdot Q \cdot A_{d}}{\tau_{R P E}^{2}(\lambda) \cdot R_{R P E}(\lambda)}
$$

In Eq. (4), all the parameters related to the incident light intensity and the transmission of all the eye components anterior to the RPE layer are eliminated. 


\subsubsection{Animal experiments}

To test the capabilities of the imaging technique, we imaged the retina of albino Sprague Dawley rats (SD rats, Taconic). All the experiments were performed in compliance with the ARVO Statement for the Use of Animals in Ophthalmic and Vision Research and with the guidelines of the Florida International University's Institutional Animal Care and Use Committee.

The animals were anesthetized by intraperitoneal injection of a cocktail containing ketamine $(54 \mathrm{mg} / \mathrm{kg}$ body weight) and xylazine $(6 \mathrm{mg} / \mathrm{kg}$ body weight). The pupil was dilated with a $10 \%$ phenylephrine solution. Drops of artificial tears were applied during the imaging to prevent corneal dehydration and cataract formation. The rat was restrained in an animal mount, which was placed on a five-axis platform.

For FA imaging, Fluorescite $10 \%(100 \mathrm{mg} / \mathrm{ml}$ fluorescein, $50 \mathrm{mg} / \mathrm{kg}$ body weight) was injected through the tail vein after the rat was stabilized and the eye was aligned with the probe light. Then the simultaneous images were captured.

\subsubsection{Phantom construction}

To further test the imaging system and the theoretical models, we built a phantom to simulate light attenuation by tissues anterior to the RPE (Fig. 3-2(a)). The phantom consisted of three components: a plastic weighing dish as the container, fluorescein sodium solution in the container to simulate lipofuscin, and a transparent polystyrene membrane to cover the fluorescent dye solution and control the thickness of the sample. A set of ND filters (nominal OD values of $0.1-0.5$ ) was placed in front of the phantom for light attenuation. 


\subsection{Results}

\subsubsection{Phantom test}

We built a phantom to obtain proof-of-concept of the system design and theoretical models using an ND filter to simulate signal attenuation. The phantom setup has two boundaries in the OCT cross-sectional image: the 1 st is the polystyrene membrane and the 2 nd is the surface of the weighing dish. OCT and fluorescent images were simultaneously acquired. The OD value of the sample was measured to be $1.26 \pm 0.02$. By segmentation, we obtained the intensity of the OCT reflection from the 2nd boundary (Fig. 3-2, red curve). As the OD value increases, both the fluorescent (Fig. 3-2(b), blue curve) and OCT signals decreased. But the normalized fluorescent signals (Fig. 3-2(b), Fluorescence/OCT, green curve) calculated using Eq. (4) showed only a gradual and linear decrease, which was caused by accumulated bleaching of the dye during the measurements as the measurements were taken sequentially from low to high OD values. Each measurement causes partial bleaching of the dye and the effect accumulates unless the time interval among measurements was sufficient for the dye to recover. It is clear that the normalized AF signals are independent of the OD values of the ND filter. As we noticed, the $\mathrm{OD}=0.3$ filter had a large deviation from the nominal OD value, which made the measured AF and OCT signal curves "nonlinear" (Fig. 3-2(b), red and blue curves), and yet the linearity of the normalized AF curve was not affected (Fig. 3-2, green curves).

The results of these experiments demonstrated that OCT signals can be used as an internal reference to minimize the effects of media anterior to the RPE, and the imaging system functions as expected. 
(a)

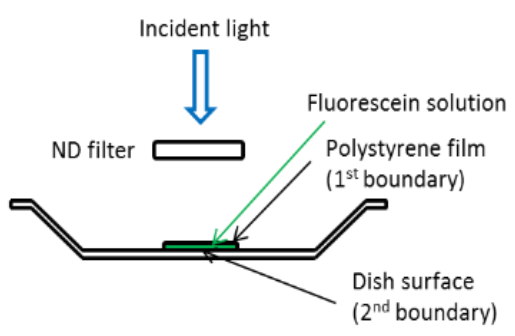

(b)

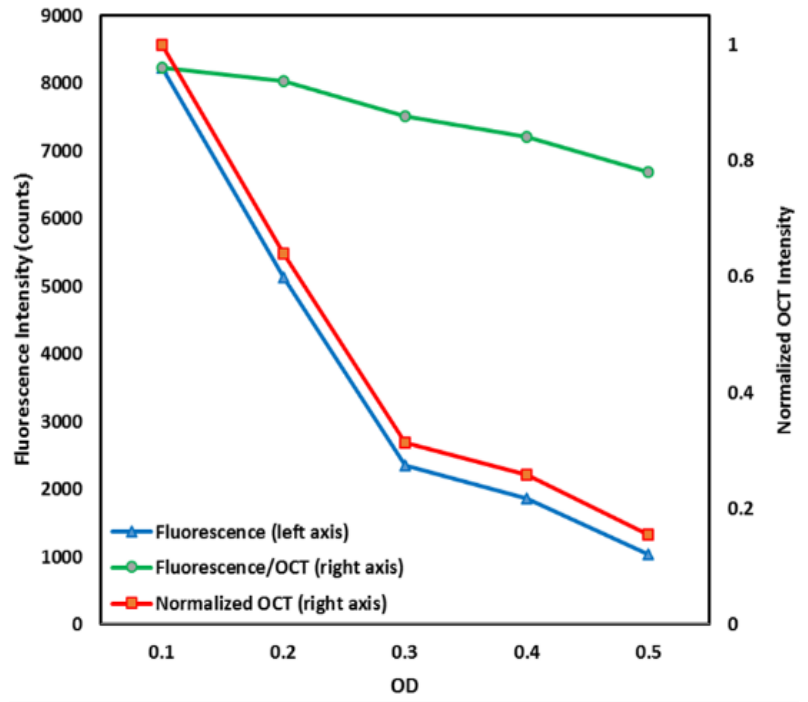

Figure 3-2 Phantom simulation experiment. (a) Schematic of the phantom; (b) The AF/OCT (the AF intensity divided by the $2^{\text {nd }}$ boundary OCT intensity, green) signals were not influenced by the OD values of the ND filter, unlike the raw AF signals (blue) and OCT signals (red). The linear decrease of AF/OCT was due to accumulated bleaching of the dye.

\subsection{2 in vivo simultaneous VIS-OCT and retina AF imaging}

The system was tested in SD rats. Figure 3-3 shows an example of the simultaneously acquired VIS-OCT (Fig. 3-3(a) and 3-3(c)) and AF (Fig. 3-3(b)) images of the retina of an animal (age: 12 months; body weight: 500 g). Fig. 3-3(a) is the projected OCT fundus image generated from the acquired 3D VIS-OCT dataset ${ }^{75}$. Fig. 3-3(c) is the VIS-OCT cross-sectional B-scan image, the location of which is marked as a dotted line in Fig. 33(a) and 3-3(b). The OCT dataset consists of 512 (horizontal) $\times 128$ (vertical) A-scans. Since the OCT and AF images are generated from the same photons, we can see that Fig. 3-3(a) and 3-3(b) are precisely registered. 

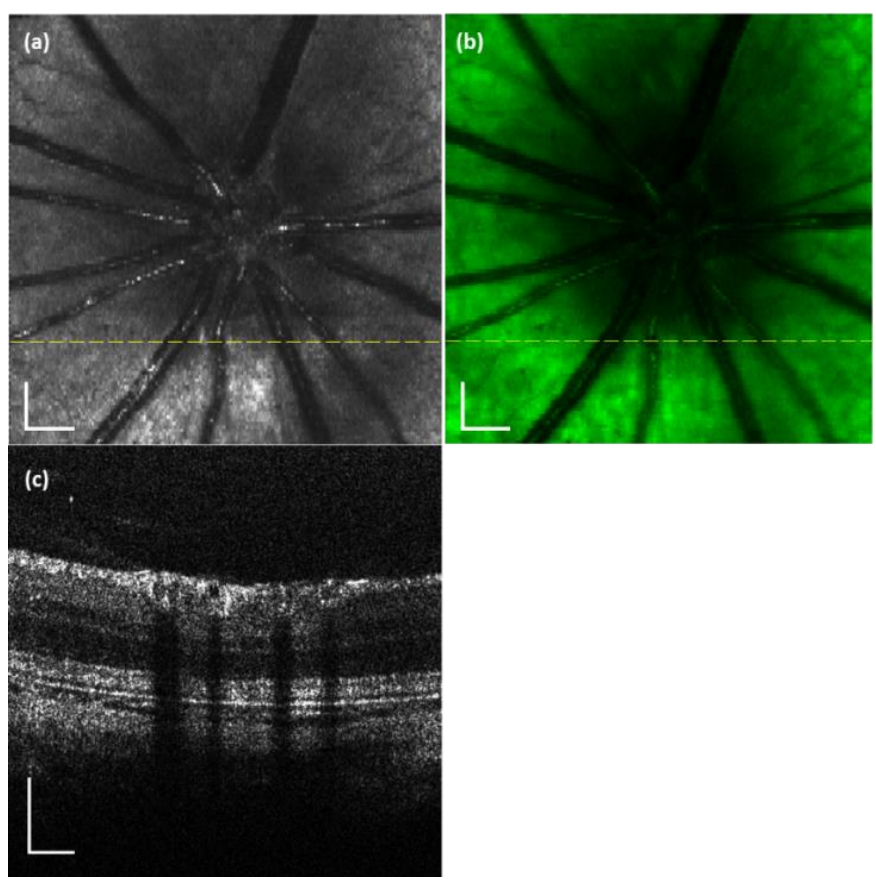

Figure 3-3 VIS-OCT and AF images simultaneously acquired from a rat retina in vivo. (a) OCT fundus image; (b) AF image; (c) OCT B-scan image. The dotted line in the OCT fundus image marks the location of the OCT B-scan image. Bar: $200 \mu \mathrm{m}$
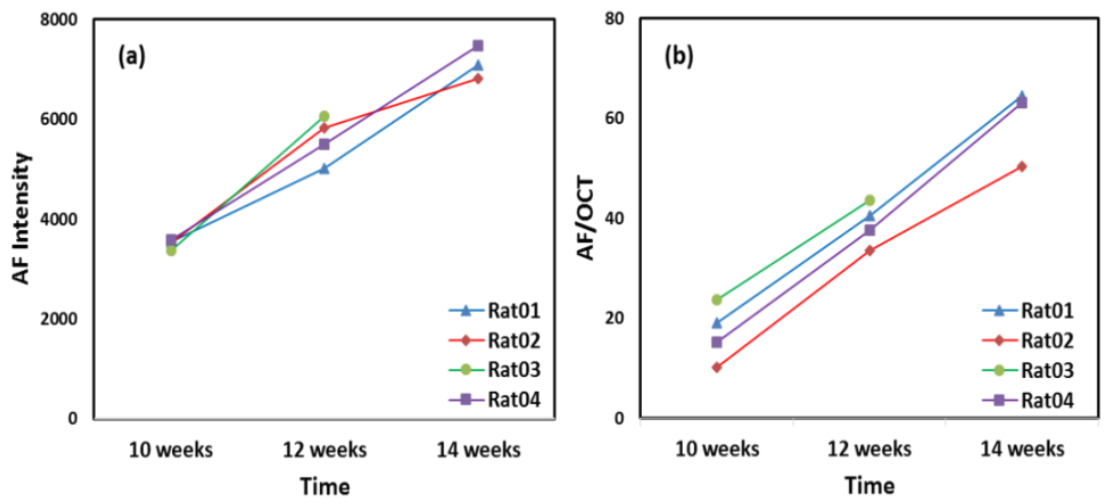

Figure 3-4 The original AF intensities (a) and the normalized AF intensities (b) of four rats followed for a period of four weeks. Normalization was performed using the RPE image intensities calculated from the segmented OCT images.

In our previous studies ${ }^{70}, 4 \mathrm{SD}$ rats (10-week-old) were imaged every two weeks (one rat died after the second imaging) to monitor their AF in the retinas for 4 weeks. The imaging conditions, including the focusing and the power of the probe light, the position of the reference arm, and the depth of the retina in the OCT image, were kept the same in each 
imaging experiment. The mean AF intensity counts over the entire imaged area of the 4 rats at different time points are shown in Fig. 3-4(a). The accumulation of lipofuscin in the RPE cells was evidenced by the increased AF image intensities over time.

The mean AF intensity counts were then normalized with the RPE OCT signal intensities obtained by manually segmenting the RPE layer and averaged over the entire imaged area. The normalized AF intensity is calculated by using Eq. (4). From the raw mean AF intensity counts, we can see significant increases over the 4-week period, but the rate of increase in each animal differs significantly. When the normalized data were used (Fig. 34(b)), the rates of increase among animals are quite similar. Note that the "levels" of $I_{A F}$ in Fig. 3-4(b) between animals are not normalized to a common reference, and thus they cannot be compared quantitatively if not all the imaging conditions including the eye conditions are known. The Y-axis scale in Fig. 3-4(b) has no specific physical meaning in this case.

All rats in the experiment were of the same age and housed under the same lighting conditions. It is reasonable to believe that the rate of lipofuscin accumulation is similar for all the rats. Before normalization using Eq. (4) the rate of AF intensity increase, thus the rate of lipofuscin accumulation, is significantly different among the different rats. After normalization using Eq. (4), the AF intensities of different rats increase over time at a similar rate. Further, as we mentioned earlier, literature shows a linear correlation between aging and lipofuscin accumulation ${ }^{76}$ which is better observed in the results using Eq. (4) for normalization. 


\subsection{3 in vivo simultaneous VIS-OCT and retinal FA}

Since the system is capable of simultaneous OCT and AF imaging. We wondered if we can test this capability to image FA. Due to the relatively slow imaging speed, which is mainly limited by the bandwidth of the PMT and the line rate of the CCD camera used in the current system, we could not control which FA phase to capture. This limitation could be removed with a detector of higher speed to capture all the different FA phases.

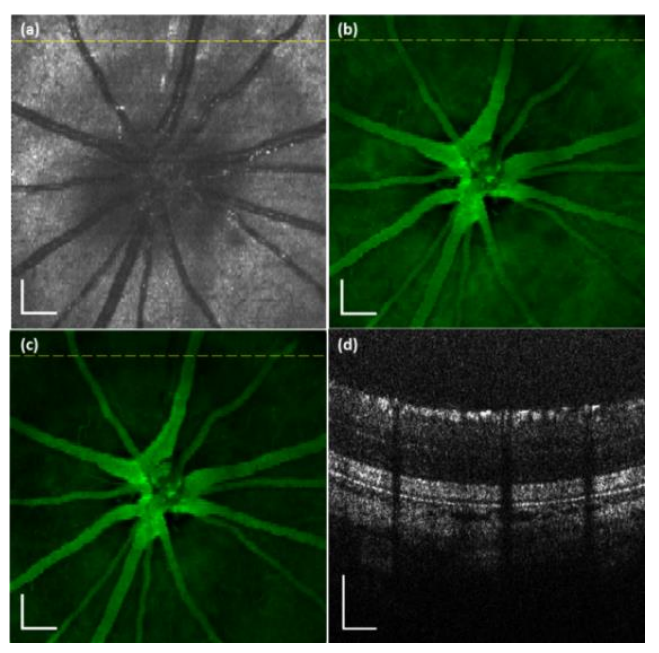

Figure 3-5 VIS-OCT and AF images simultaneously acquired from a rat retina in vivo. (a) OCT fundus image; (b) AF image; (c) AF/OCT compensated fundus image; (d) OCT B-scan image. The dotted line in the OCT fundus image marks the location of the OCT B-scan image. Bar: $200 \mu \mathrm{m}$

The simultaneously acquired VIS-OCT and FA images of the retina of an SD rat are shown in Fig. 3-5. Spatial registration is guaranteed between the OCT and FA images because they were generated by the same photons. The FA image (Fig. 3-5(b)) was normalized with the projected OCT (Fig. 3-5(a)) intensities pixel by pixel (Fig. 3-5(c)) to produce better contrast for the retinal blood vessels. This contrast enhancement of the retinal blood vessels is achieved by suppression of the fluorescence signals from the choroidal blood vessels. 


\subsection{Discussion}

We have demonstrated the capability of a multimodal imaging system for simultaneous OCT and fluorescent imaging with the same broadband light source. The fluorophores could be endogenous or exogenous. The major limitation of the current system is the imaging speed, which made the system unable to image the early FA phases.

This system can be used to quantitatively imaging retinal AF, a measure of fluorophores mainly contained in the lipofuscin granules in the RPE ${ }^{64,76}$. AF was first detected in a vitreous fluorophotometry study ${ }^{77}$. The major fluorophore in lipofuscin granules and the major fluorophore of retinal $\mathrm{AF}$ is believed to be A2E (N-retinyl-N-retinylidene ethanolamine), a byproduct of the photoreceptor visual cycle ${ }^{78,79}$. The RPE is a monolayer of pigmented cells located between photoreceptors and the choroid ${ }^{80}$. It helps to enhance vision by absorbing scattered light with melanin, maintains the normal function of photoreceptors, including regenerating 11 -cis retinal in the visual cycle ${ }^{81}$ and phagocytosis of the shed photoreceptor outer segment tips ${ }^{82}$. Since lipofuscin has been implicated in retinal diseases such as AMD and Stargardt diseases, quantitatively measuring AF is of significant clinical value.

Quantification of absolute retinal AF intensities is important for $\mathrm{AF}$ to be used in clinical research and patient care, without which it is difficult to evaluate retinal AF images from different patients or even between successive images from the same patient. However, the

available AF imaging technologies are not able to quantify absolute AF intensities. And this is the major hurdle for AF imaging to be widely utilized. We believe that to achieve 
quantification of absolute $\mathrm{AF}$ intensities, we need to have an internal reference to measure the true intensities occur at the RPE, and a fluorescent standard with known intensity as an external reference similar to the reference fluorescent target used by Delori and co-workers ${ }^{16}$. Our technique of simultaneous VIS-OCT and retinal AF imaging by using the same light source is unique in providing an internal reference to eliminate the attenuation effects of the media anterior to the RPE. Thus, our system of simultaneous OCT and AF in combination with a fluorescent standard reference should be able to achieve quantification of absolute AF imaging for clinical application.

The system is also capable to simultaneously acquire OCT and FA images. For potential clinical application, the system will need faster detectors to capture different phases of FA. The multimodal VIS-OCT system described here is one of the several systems we have developed, including simultaneous photoacoustic microscopy (PAM) and autofluorescence microscopy ${ }^{83,84}$, simultaneous OCT and PAM ${ }^{85,86}$, and simultaneous OCT and retinal AF ${ }^{60,70}$. Further studies to optimize these systems in disease models could lead to the clinical application of these navel technologies.

To get the result of Eq. 4 we made an assumption that $\tau_{p r e}$ and $R_{p r e}$ are the same for excitation light and the fluorescent. Although the assumption was made for simplifying the mathematical model, it is based on a reasonable analysis. In the anterior segments, light attenuation in the visible spectrum is mainly determined by the scattering coefficients of the tissues and light absorption can be ignored. Thus $\tau_{p r e}$ is close to unity for the anterior segments. Light transmission is mainly determined by $\left(1-R_{p r e}\right)$, which slightly increases with the wavelength in the visible spectrum ${ }^{15}$. For the retinal tissues anterior to the RPE, 
light absorption is mainly caused by hemoglobin in the retinal blood vessels, whose overall absorption coefficient increases with the wavelength in the spectral range from $480 \mathrm{~nm}$ to $570 \mathrm{~nm}$. In the meantime, in the same spectral range light scattering decreases with wavelength. As a result, the overall effect of the ocular tissues anterior to the RPE layer (1$\left.R_{\text {pre }}\right) . \tau_{\text {pre }}$ may remain relatively constant for the excitation and AF light. In future studies, we will design experiments to test the accuracy of this assumption and improve the model if the systematic error is introduced by the assumption. In addition, AF imaging is usually performed under light conditions, so the effect of the absorption spectrum by the visual pigment can be ignored.

In the phantom study, higher dye concentration will reduce the signal intensity from the second boundary. When the concentration of the dye is higher than a certain level, determined by the sensitivity of the OCT, the second boundary of the phantom could no longer be reliably imaged. In such an extreme situation, the normalization method cannot be used. We recognize that there are limitations of the current method. It seems unlikely that the limitations would be an issue in practice as we haven't seen the situation in which the RPE layer cannot be revealed in our in vivo imaging experiments on both albino and pigmented rats.

For in vivo imaging applications $\tau_{R P E}$ and $R_{R P E}$ are functions of the concentrations of both melanin and lipofuscin in the RPE layer. Lipofuscin and melanin exist in the RPE cells as granules. Thus, the increase in lipofuscin will reduce $\tau_{R P E}$ but will increase $R_{R P E}$. Lipofuscin accumulates during aging, but melanin will probably decrease ${ }^{87}$ and the decrease in 
melanin would contribute inversely to $\tau_{R P E}$ and $R_{R P E}$ How these combined effects influence $\tau_{R P E}$ and $R_{R P E}$ cannot be easily answered and needs further investigation.

The normalization method is designed to remove the attenuation effects of the tissues anterior to the RPE layer as well as the effects of factors like misalignment and to retain only the factors describing the optical properties of the RPE. Experiments with phantoms and in vivo studies on rats have demonstrated the promising potentials of the method to reach this goal. However, more specifically designed experiments are needed to examine the exact meanings of the method and its capabilities and limitations in extracting the true AF intensities of the RPE lipofuscin.

\subsection{Conclusion}

In conclusion, we have, for the first time, achieved simultaneous VIS-OCT and fluorescence imaging with a single broadband light source targeting both endogenous and exogenous fluorophores. This multimodal imaging system takes a significant step toward the quantification of true retinal AF intensities. Further optimizing this system would make it a valuable tool for clinical and research applications. 


\section{CHAPTER 4 Quantification of VIS-OCT and FAF for elimination of acquisition parameter dependence}

\subsection{Introduction}

Lipofuscin, a complex mixture of partially digested lipids and protein components in the retinal pigment epithelium (RPE) cells, is a major source of fundus autofluorescence (FAF) 68,88. It accumulates with age and is implicated in age-related macular degeneration (AMD) and Stargardt disease ${ }^{89}$. FAF hence is a natural biomarker that carries the information of lipofuscin content, and quantification of FAF signal could be used to assess the amount of lipofuscin in the RPE for diagnosis and monitoring disease progression ${ }^{90-97}$

FAF imaging has been used in ophthalmology clinics for many years. For example, hyperautofluorescence is positively correlated with the progression of AMD and Stargardt's macular dystrophy ${ }^{98,99}$. In the case of geographic atrophy (GA), the late stage of dry AMD, advanced RPE alterations exhibit clinically recognizable patterns of hyperautofluorescence ${ }^{100}$, which is positively correlated with the rate of GA progression and can be analyzed semi-automatically with newly developed software by non-expert graders 101.

However, the currently available technologies are not capable to measure standardized FAF intensity ${ }^{13}$. The measured FAF signal by the currently available technologies could be affected by the excitation light intensity, detector sensitivity and gain of the instrument used. In addition, and importantly, signals are attenuated by the ocular tissues anterior to 
the RPE, especially by the lens, and the attenuation cannot be measured directly. The ocular properties of tissues anterior to the RPE could be significantly different among individuals, and it changes over time in the same person. Thus, it is difficult to compare images obtained by the currently available technologies from the same person over time, or from different individuals, which hinders the clinical usefulness of FAF images ${ }^{34,102}$. It is a challenge to obtain the absolute intensity of FAF.

To compare the two different FAF images taken at different times or from different individuals, the intensity of the FAF images should be standardized and free of the effects of instrumental variation and attenuation by the ocular tissues in the optical path before the RPE. Standardized FAF images can be achieved by measuring the absolute FAF intensities, or the intensity of each pixel in a FAF image is referenced to an intensity standard. The issue of FAF intensity calibration and instrument fluctuation compensation has been addressed by Delori et al. ${ }^{16}$ by using a fluorescent target with known emission efficiency in the intermediate retinal imaging plane as a reference. However, how to accurately compensate for the signal attenuation by tissues in the optical path before the RPE remains a challenge.

We reasoned that compensation for signal attenuation by ocular tissues can be achieved by combining visible-light optical coherence tomography (VIS-OCT) and confocal FAF microscopy that share a common light source. Previously we explored simultaneous VISOCT and FAF imaging using first a frequency-doubled Ti: sapphire laser ${ }^{60}$, and later a supercontinuum laser with a filtered output centered at $488 \mathrm{~nm}$, an AF excitation wavelength used in current FAF imaging systems ${ }^{70}$. Recently, we demonstrated that in a 
dual-modal VIS-OCT and FAF system, the simultaneously acquired VIS-OCT images can be used to compensate for the power variations and attenuation factors for the FAF images ${ }^{31}$. In such a dual-modal imaging system, the photons from the light source go through the same optical pathways and are attenuated by the same media to generate signals for OCT and FAF. Therefore, the OCT signals can be used to compensate for both the illumination variation and the signal attenuation by the ocular tissues. A unique feature of the technique is that the OCT and FAF images are intrinsically registered because the images in both modalities are generated by the same group of photons. However, a missing link in this system is the relationship between the amplitude of the OCT signals and the reflectivity of the sample boundaries, e.g. the RPE of the retina.

Here, we report a new development of the VIS-OCT based multimodal imaging technology for quantitative FAF imaging. In the new system, we introduced two reference targets in the intermediate retinal imaging plane and thus the two imaging modalities are correlated quantitatively. One target has known reflectivity for OCT reference, and the other has known fluorescence efficiency for FAF reference. The fluorescence reference target has similar fluorescence characteristics of the target used by Delori ${ }^{16}$. The reflectivity reference has the same plastic substrate as the fluorescence target with known refractive index and emission spectrum out of the major detection wavelengths of the FAF. The system uses a single broadband light source with a center wavelength of $480 \mathrm{~nm}$ to provide simultaneous OCT and retinal AF imaging. True FAF signal intensities can be obtained by normalizing the OCT and FAF images from the eye to the corresponding signals from the reference standards to compensate the instrument fluctuations, including laser power and 
detector sensitivity changes, and the signal attenuation by the media anterior to the RPE can be compensated by AF/OCT calculation. The system has been successfully tested on phantoms, and in rat retinas in vivo.

\subsection{Materials and Methods}

\subsubsection{Imaging system}

A schematic of the multimodal imaging system is shown in Fig. 4-1. The system consists of two spectral-domain OCTs in the NIR and visible spectrum, respectively. The NIR-OCT is used only for alignment to reduce visible light exposure and avoid additional bleaching effects to the fluorophores. The VIS-OCT consists of a supercontinuum laser (SC, model: EXB-6, SuperK EXTREME, NKT Photonics, Denmark) with a variable band-pass filter (selected center wavelength: $480 \mathrm{~nm}$, bandwidth: $30 \mathrm{~nm}$ ). The output VIS light is coupled into the source arm of a single-mode optical fiber-based Michelson interferometer. The NIR-OCT uses a superluminescent diode as the light source (SLD-37-HP, center wavelength: $840 \mathrm{~nm}$, bandwidth: $50 \mathrm{~nm}$, Superlum, Russia). The NIR light is coupled to another fiber-based Michelson interferometer after passing through an optical-fiber isolator. After exiting the optical fibers in the sample arms, both the NIR and VIS light are collimated and combined by two dichroic mirrors (DM1: DMLP505, Thorlabs, and DM2: NT43-955, Edmund Optics). The combined light beam is scanned and delivered to the eye by a combination of a relay lens (L1, $\mathrm{f}=75 \mathrm{~mm}$, achromatic) and an ocular lens (L2, Volk lens, 60D). Two standard reference targets for fluorescence (Fluor-Ref, Microscopy Education, Texas Red) and reflectance (Fluor-Ref, Microscopy Education, DAPI) are placed in the intermediate retinal imaging plane covering about $14 \%$ of the lower part of 
the field of view. The fluorescence reference target is optically cemented to a Neutral Density filter (OD: 10, absorptive type) to reduce the reference fluorescence signal to the range of the FAF of rodent retina. There are two VIS-OCT reference arms for retinal and reference-target imaging, respectively. After exiting the optical fiber in the reference arm the VIS light is collimated and split into two beams by a non-polarizing beam splitter (NBS). The two light beams are reflected back by two mirrors, recombined by the NBS, and coupled back into the optical fiber. The two reference arms have different path lengths, the longer path length for imaging the retina and the shorter path length for imaging the reference target. A computer-controlled shutter (SH1, Thorlabs) was used in the reference arm with shorter path length to synchronize with the reference-target imaging. During each raster scan, the shutter was closed to block the reference light for the reference target when the retina was imaged. When the probe light was scanned on the reference target the shutter was opened to allow the reference target to be imaged with the VIS-OCT. The VIS light power was $500 \mu \mathrm{W}$ before entering the eye while NIR light power is $600 \mu \mathrm{W}$.

In the detection arms, reflected light from the sample and reference arms of the two OCT systems is detected by two spectrometers (SPEC1-2). The VIS-OCT and NIR-OCT use spectrometers with the same parameters described in our previous publication ${ }^{31}$. The axial resolution of the VIS-OCT in the air was measured to be $6 \mu \mathrm{m}$ with a sensitivity of $85 \mathrm{~dB}$. The OCT signal roll-off was measured to be $-8 \mathrm{~dB}$ in a depth of $2 \mathrm{~mm}$ and was compensated in image processing.

FAF is detected by a PMT module (H10723-20, Hamamatsu). The fluorescence photons pass through the two dichroic mirrors as well as a long pass filter (FGL515M, cut-on 
wavelength: $515 \mathrm{~nm}$, Thorlabs), and are then focused to a $25 \mu \mathrm{m}$ pinhole by an achromatic doublet with a focal length of $30 \mathrm{~mm}$. The outputs of the PMT are digitized by a multifunction data acquisition board (DAQ, PCIe-6361, National Instruments) at a sampling rate of $2 \mathrm{Ms} / \mathrm{s}$ and a sampling length of 80 points. The amplitudes of the FAF signal of the 80 points were averaged to form a single pixel of the FAF image. Synchronization of the FAF data acquisition, scanning of the galvanometer scanner and the OCT image acquisition was controlled by the multifunction DAQ board. The imaging speed of both the OCT and FAF was set to 24,000 A-lines/s limited by the speed of the line-scan CCD camera in the spectrometer.

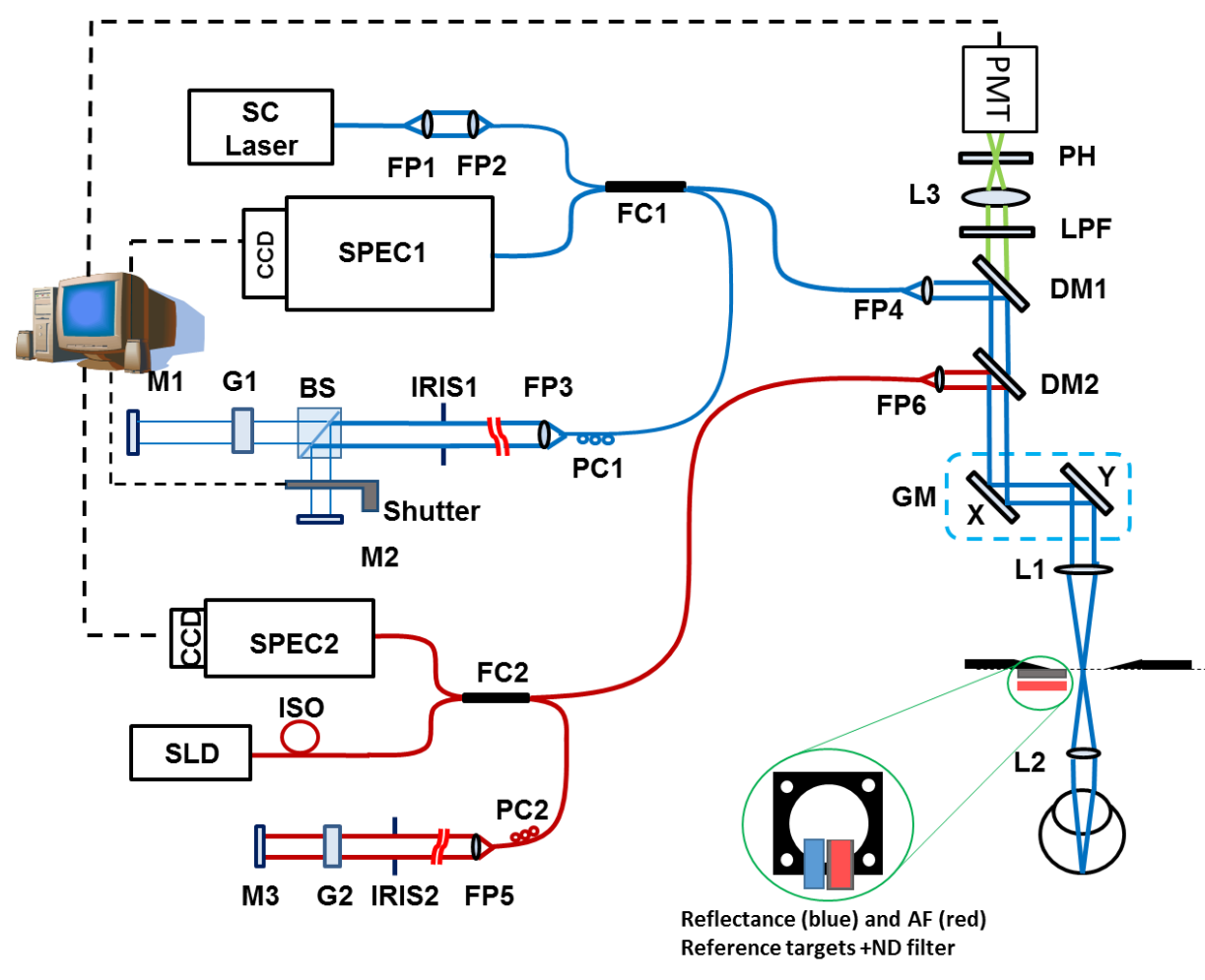

Figure 4-1 Schematic of VIS-OCT-FAF with two references: VIS-OCT (blue), NIR-OCT (red) and AF (green). SLD: Superluminescent Diod; SC: Supercontinuum; PMT: Photo Multiplier Tube; SPEC1-2: Spectrometer; ISO: Isolator; M1-3: Reference arm mirrors; IRIS1-2: iris; G1-2: BK7 glass plates; BS: beam splitter; FC1-2: fiber coupler; FP1-6: collimation fiber ports; PC1-2: polarization controller; GM: 
galvanometer scanner; DM1-2: dichroic mirrors; PH: pinhole, L1-3: lens; LPF: long-pass filter; ND filter (OD: 10).

\subsubsection{Mathematical model of OCT reflectance and AF signals}

The OCT signal in the time domain from a sample can be expressed as

$$
I=R_{r} I_{r}+R_{s} I_{s}+2 \sqrt{R_{r} I_{r} R_{S} I_{s}} \int G(v) \cos (\Delta \phi) d v=I_{D C}+I_{A C}
$$

where $I_{S}$ and $I_{r}$ are the incident light intensity in the sample and reference arms; Rs and $R r$ are the reflecting coefficients of the sample and the reference mirror; $G$ is the spectral power density of the light source; $\mathrm{I}_{\mathrm{DC}}$ and $\mathrm{I}_{\mathrm{AC}}$ are the $\mathrm{DC}$ and $\mathrm{AC}$ components of the detected OCT signal:

$$
\begin{gathered}
I_{D C}=R_{r} I_{r}+R_{s} I_{s} \\
I_{A C}=2 \sqrt{R_{r} I_{r} R_{s} I_{s}} \int_{0}^{\infty} G(v) \cos (\Delta \phi) d v
\end{gathered}
$$

We usually have:

$$
\begin{gathered}
R_{S} I_{s} \ll R_{r} I_{r} \\
I_{D C} \approx R_{r} I_{r}
\end{gathered}
$$

The OCT image, which is generated from the AC portion of the detected signals, is proportional to the square root of the sample reflectance (Eq. (3)). We thus have:

$$
R_{O C T}=I_{A C}^{2} / I_{D C} \propto R_{S} I_{S}
$$


where ROCT represents the OCT reflectance signal. The OCT signals from the RPE can be extracted by using image segmentation. For the OCT signals of the RPE, $\mathrm{I}_{\mathrm{s}}$ in Eq. (5) is the light intensity incident onto the RPE layer, which contains the attenuation information from the anterior segments of the eye down to the retinal tissues before the RPE. The same incident light generates both the AF and the OCT signals. According to the ocular reflectance model proposed by van de Kraats et al, reflectance from the retina can be categorized into reflectance from layers of the pre-RPE, the RPE, and the post-RPE ${ }^{73,74}$. Through a detailed analysis of the OCT and AF signals and in reference to the previous studies ${ }^{31,16}$, we can calculate the following ratio:

$$
\begin{gathered}
\frac{I_{F A F}}{I_{R A F}}=\frac{I_{0} \tau_{\text {pre }}^{2}(\lambda)\left[1-\rho_{\text {pre }}(\lambda)\right]^{2} \xi_{R P E} A_{d} \frac{\pi}{4} \alpha^{2}}{I_{0} \xi_{R} A_{d} \frac{\pi}{4} \alpha^{\prime 2}}=\frac{\tau_{\text {pre }}^{2}(\lambda)\left[1-\rho_{\text {pre }}(\lambda)\right]^{2} \xi_{R P E} \frac{\pi}{4} \alpha^{2}}{\xi_{R} \frac{\pi}{4} \alpha^{\prime 2}} \\
\frac{R_{O C T-R P E}}{R_{O C T-R}}=\frac{I_{0} \tau_{\text {pre }}^{2}(\lambda)\left[1-\rho_{\text {pre }}(\lambda)\right]^{2} \rho_{R P E}(\lambda) \frac{\pi}{4} \alpha^{2}}{I_{0} \rho_{R}(\lambda) \frac{\pi}{4} \alpha^{\prime 2}}=\frac{\tau_{\text {pre }}^{2}(\lambda)\left[1-\rho_{\text {pre }}(\lambda)\right]^{2} \rho_{R P E}(\lambda) \frac{\pi}{4} \alpha^{2}}{\rho_{R}(\lambda) \frac{\pi}{4} \alpha^{\prime 2}}
\end{gathered}
$$

where, $I_{F A F}$ and $I_{R A F}$ are the fluorescence intensities of the retina and the reference target, respectively. ROCT-RPE and ROCT-R are the OCT signal intensities of the RPE and the reference targets, respectively, calculated with Eq. (5). $I_{0}$ is the light intensity incident into the eye. $\tau, \rho, \xi$ and $A_{d}$ represent transmittance, reflectance, fluorescence efficiency, and detector sensitivity, respectively. Subscribes pre, RPE and R denote the pre-RPE media, the RPE layer and, the reference standard targets. $\frac{\pi}{4} \alpha^{2}$ and $\frac{\pi}{4} \alpha^{\prime 2}$ are the solid angles that comprise the light reaching to the pinhole and hence the detector, respectively, from the retina and reference targets. The fluorescence efficiency $\xi$ is defined as the product of the fluorophore concentration $(\mathrm{C})$, the molecular quantum yield $(\mathrm{Q})$, and extinction coefficient 
$(\varepsilon),(\xi=C \times Q \times \varepsilon \times d){ }^{103}$. Assuming the same attenuation of the pre-RPE media for the excitation and emission wavelengths, we can calculate the ratio:

$$
\frac{I_{F A F} / I_{R A F}}{R_{O C T-R P E} / R_{O C T-R}}=\frac{C_{L} Q_{L} \varepsilon_{L} d_{R P E} / C_{R} Q_{R} \varepsilon_{R} d_{R}}{\rho_{R P E} / \rho_{R}}
$$

where $\mathrm{C}, \mathrm{Q}, \varepsilon$, and $\mathrm{d}$ are concentration, quantum yield, extinction coefficient, and the effective detection thickness, respectively. Subscribes L and R, respectively represent lipofuscin present in RPE and Texas Red embedded in the reference target. With the known properties of the reference target, we can see that the calculated ratio is only dependent on the optical properties of the RPE and the lipofuscin concentration present in the RPE.

\subsubsection{Animal experiments}

To assess the capability of the imaging technique and quantification method, we imaged both albino Sprague Dawley rats (SD rats, two age groups: five 2-months-old and six 14months-old) and pigmented Long Evans rats (five 12-months-old). The experiments were conducted in agreement with the ARVO Statement for the Use of Animals in Ophthalmic and Vision Research and with the guidelines of the Florida International University's Institutional Animal Care and Use Committee.

Intraperitoneal injection of a cocktail of ketamine $(54 \mathrm{mg} / \mathrm{kg}$ body weight $)$ and xylazine $(6 \mathrm{mg} / \mathrm{kg}$ body weight) was used to anesthetize the animals. Then the pupil was dilated with a drop of $10 \%$ phenylephrine solution. To prevent corneal dehydration and cataract formation a hard contact lens was put on the eye. The rat was restrained in an animal mount, which was placed on a five-axis platform. 


\subsubsection{Phantom study}

To validate the theoretical model, we need to check the linearity of RocT/ ROCT-R vs Rs, and the linearity of $\mathrm{I}_{\mathrm{FAF}} / \mathrm{I}_{\mathrm{RAF}}$ vs fluorescence efficiency. The validation was accomplished by imaging a model eye consisting of an aspherical lens $(\mathrm{f}=15 \mathrm{~mm})$ and a master reference target (Microscopy Education, Fluor Ref slide: Green excitation (FITC)), which was used to simulate the retina. A set of five neutral density (ND) filters (NEK01, Thorlabs,) with optical densities of $\mathrm{OD}=0.1,0.2,0.3,0.4$, and 0.5 was used to simulate the attenuations before the retina. Fig. 4-2(d) shows a schematic of the model eye in the phantom study. Imaging was performed with different powers of the light source and different detector sensitivities.

The $\mathrm{R}_{\mathrm{OCT}}$ signals of the master reference in the model eye were calculated from their OCT images and then divided by ROCT-R calculated from the OCT images of the reflectance reference target. The calculated $\mathrm{R}_{\mathrm{OCT}} / \mathrm{R}_{\mathrm{OCT}} \mathrm{R}$ is expected to be independent of the power of the light source.

Similarly, the AF signals of the master reference in the model eye were normalized to the AF signals of the fluorescence reference target. The result is expected to be independent of the detector sensitivity and light power.

Finally, the ratio of normalized AF (qAF) to normalized OCT (qOCT), qAF/qOCT, is calculated, which is expected to be independent on the light power, the sensitivity of the detector, and the attenuation before the model eye. 


\subsubsection{Standard reference targets characterization}

The fluorescence efficiency of the reference target was measured with both a commercial fluorescence microscope and our AF-OCT system by using a fluorescein solution with known concentration. The quantum yields of Texas Red and fluorescein we used for the characterization are 0.66 and 0.92 , respectively, according to equivalent Alexa Fluor dyes (ThermoFisher Scientific). We can calculate the fluorophore concentration of the reference

target by using $\frac{\xi_{\text {Fluorescein }}}{\xi_{\text {Texas Red }}}=\frac{C_{F} Q_{F} \varepsilon_{F} d_{f}}{C_{T} Q_{T} \varepsilon_{T} d_{T}}$. The effective thickness in our measurement is limited by the axial resolution of the confocal fluorescent microscope, which remains the same for both Fluorescein and Texas Red imaging. The calculated concentration of Texas Red is $0.01 \mathrm{mg} / \mathrm{ml}$.

Reflectance of the standard reference for OCT was also measured. The target was made of PMMA, which has a reported refractive index of $\mathrm{n} \cong 1.49$, resulting in a theoretical reflectance of $\mathrm{R} \cong 0.039$. The actual refractive index was measured with OCT by calculating the ratio of Optical Distance/Actual thickness. Our measurements showed that $\mathrm{n}=$ 1.53 resulting in a theoretical reflectance of $\mathrm{R}=0.044$.

Reflectance of the target was also measured by using a beam splitter, a lens ( $\mathrm{f}=20 \mathrm{~mm}$ ) and a power meter. The intensity ratio of the reflected light to the incident light was measured to be 0.043 , which agrees with the theoretical reflectance values with a $2.5 \%$ difference. 


\subsection{Results}

\subsubsection{System calibration}

In the phantom experiments, we first investigated the influence of detector sensitivity of the system on the AF signal detection. The fluorescent intensities were measured at different detector gains (by varying the detector control voltage) to verify the capability of the technique for canceling the effects of detector sensitivity. In Fig. 4-2(a), the averaged FAF signals measured from the model eye and signals from the reference fluorescent target (RAF) were plotted against the OD values of the ND filters. At a given gain, the logarithm of the fluorescent signals from the master reference in the model eye linearly decreases with the OD values of the ND filters at a slope of -2 , which agrees with the round-trip attenuation of light through the ND filter and the listed transmission data of the ND filters. The signals from the standard fluorescent reference target are independent of the attenuation of the ND filters and remain constant. The measured signal intensities of FAF and RAF decreased with the PMT control voltage accordingly (Fig. 4-2(a)).

To verify the capability of the technique to compensate for the effects of light power, we changed the power of the light source at a constant detector gain to simulate power fluctuation. Fig. 4-2(b) shows the linear decrease of the logarithm of the FAF signal with respect to the OD values of the ND filter. As the incident light power was lowered, both the FAF and RAF signals decrease accordingly. Fig. 4-2(c) shows the calculated $\mathrm{I}_{\mathrm{FAF}} / \mathrm{I}_{\mathrm{RAF}}$ (qAF) for all the data shown in Fig. 4-2(a and b). We can see that all the effects of detector sensitivity and light power are successfully eliminated. However, the attenuation by the ND filters is not compensated in qAF calculation. This result showed that conventional 
FAF imaging techniques cannot eliminate the attenuation effects caused by tissues anterior to the RPE, even with a fluorescence reference target placed in the intermediate retinal imaging plane.
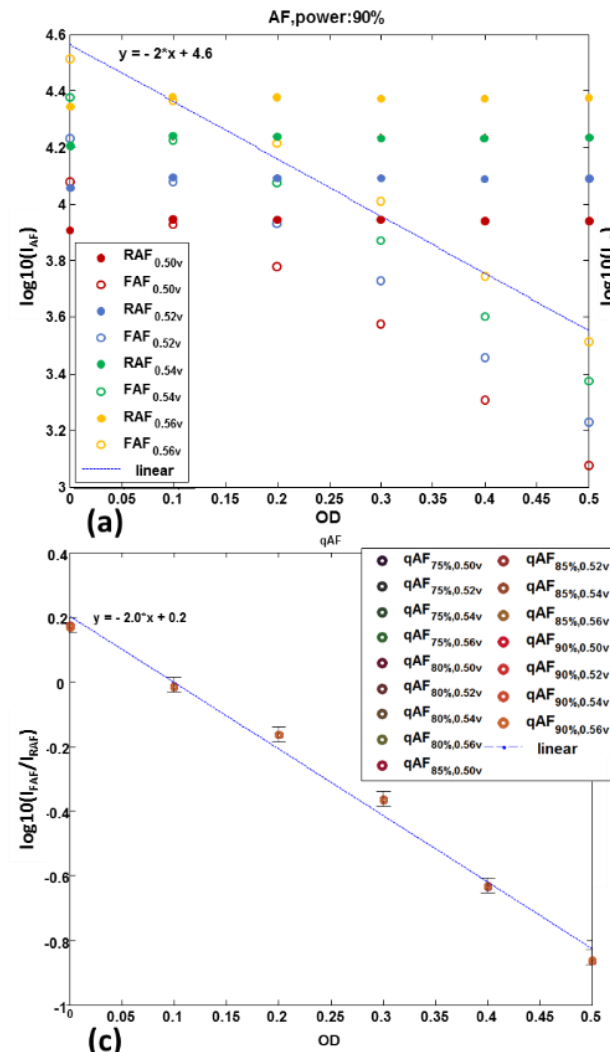

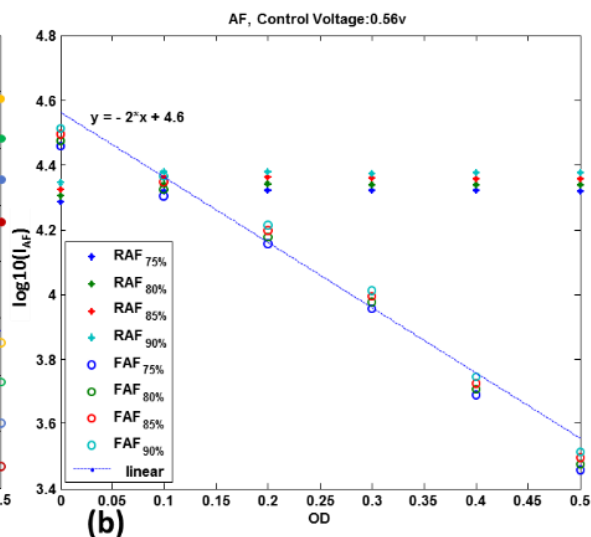

(b)

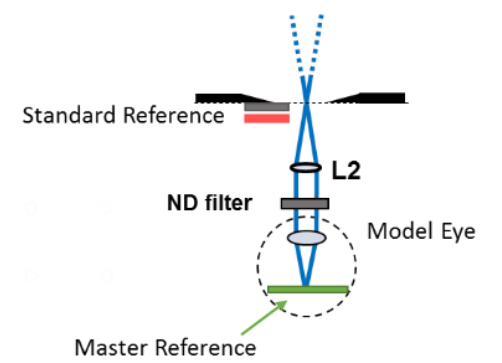

(d)

Figure 4-2 Quantitative AF signals from the model eye and the standard reference at different imaging conditions. a) AF signals vs OD value of the ND filter in front of the model eye at $90 \%$ light source power and different PMT control voltage $(0.50 \mathrm{~V}-0.56 \mathrm{~V})$; b) AF signals vs OD value of the ND filter in front of the model eye at fixed PMT control voltage $(0.56 \mathrm{~V})$ and different light source power $(75 \%-90 \%$ output power); c) The calculated $\log _{10}(\mathrm{FAF} / \mathrm{RAF})$ vs OD value of the ND filter in front of the model eye at all the different imaging conditions; d) Schematic of the model eye used in the experiments.

The simultaneously acquired OCT image of the master reference in the model eye was processed and the averaged values for each acquired image are shown in Fig. 4-3. The calculated $\log 10$ (ROCT-RPE), corresponding to the OCT signal intensities of the master reference, decreased linearly with the OD values of the ND filters (Fig. 4-3(a)). Similarly, the calculated $\log 10$ (ROCT-RPE/ROCT-R) also decreases linearly with the OD values but is 
independent of the light power (Fig. 4-3(b)). The slope of -2 of the fitted line agrees with the round-trip attenuation of the ND filters. However, the qAF/qOCT value remains relatively constant over all the experimental conditions including the different attenuation values of the ND filter (Fig. 4-3(c)). The results proved that the system is capable of compensating the attenuation of the ND filter, thus the attenuation by the media anterior to the RPE for quantifying FAF intensities.
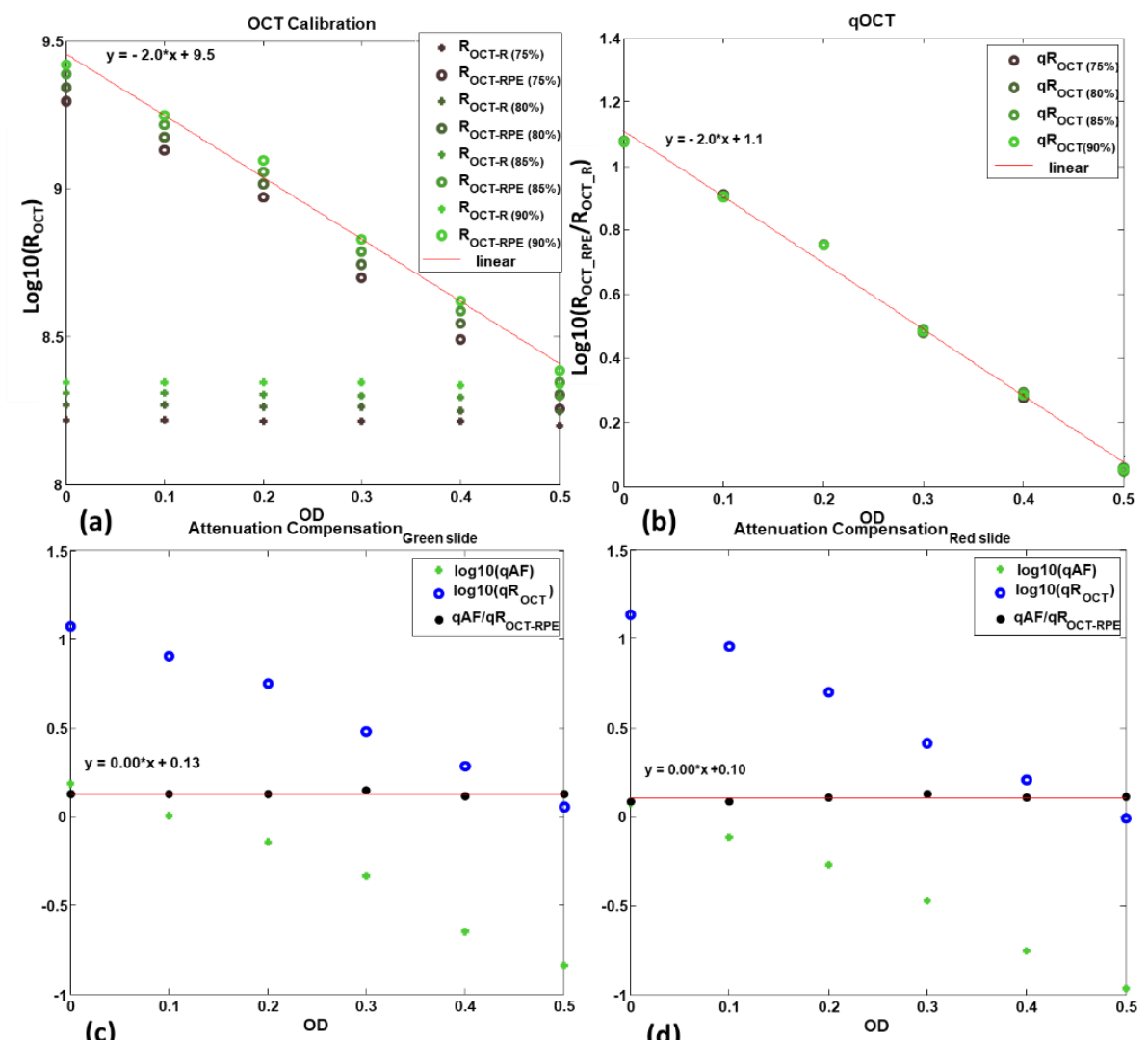

(d)

Figure 4-3 OCT reflectance signals from the model eye at different OD values of the ND filter and from the standard reference at different imaging conditions; a) The source power was changed from $75 \%$ to $90 \%$; b) OCT reflectance of the model eye normalized to the reference target (qOCT) for the data in (a); c) qAF, 
qOCT, and qAF/qOCT ratio. d) $\mathrm{qAF}, \mathrm{qOCT}$, and $\mathrm{qAF} / \mathrm{qOCT}$ ratio for the model eye with the TexasRed slide at the retinal plane.

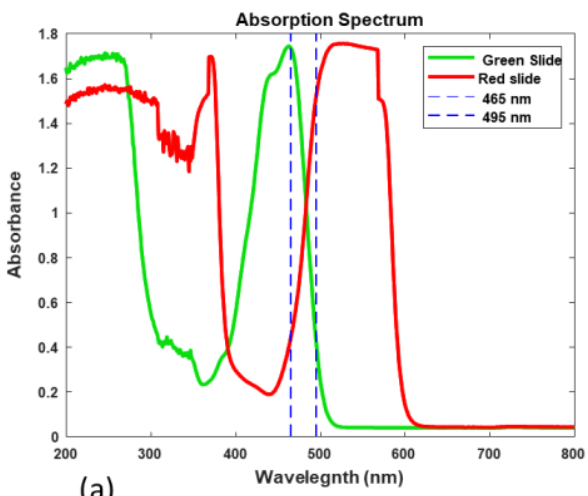

(a)

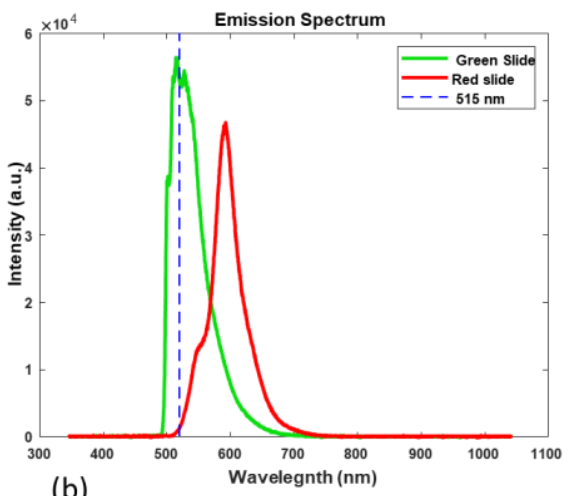

(b)

Figure 4-4 a) Absorption spectrums of green and red fluorescent slides. b) Fluorescence emission spectrums of green and red fluorescent slides.

To verify the accuracy of Eq. (8), we repeated the experiment with another fluorescent slide (Microscopy Education, Texas Red) with the same reflectance as the green master slide used before, and the results are consistent with that of the FITC slide (Fig. 4-3(d)). The measured excitation and emission spectra of the red and green slides are shown in Fig. 4-4. Considering the spectrum of the supercontinuum laser $(465 \mathrm{~nm}-496 \mathrm{~nm})$, the detection spectral range $(>515 \mathrm{~nm})$, and the quantum yields of the two fluorescent dyes $\left(\mathrm{Q}_{\text {TexasRed }}=0.66, \mathrm{Q}_{\mathrm{FITC}}=0.92\right)$, we have

$$
\frac{C_{R} \int_{465}^{495} \varepsilon_{R}(\lambda) Q_{R} \eta_{R} d \lambda}{C_{G} \int_{465}^{495} \varepsilon_{G}(\lambda) Q_{G} \eta_{G} d \lambda}=0.72
$$

where $\eta_{R}$ and $\eta_{G}$ represent the detection efficiency calculated as $\frac{\int_{515}^{800} G(\lambda) d \lambda}{\int_{346}^{800} G(\lambda) d \lambda}$, where $G(\lambda)$ is the power spectral density of the emission spectrum of the fluorescent dye. The ratio of $\frac{\left(\frac{q A F}{q O C T}\right)_{R}}{\left(\frac{q A F}{q O C T}\right)_{G}}=\frac{0.10}{0.13}=0.77$ agrees to the theory with less than a $7 \%$ deviation. However, the accurate interpretation of the values of $\mathrm{qAF} / \mathrm{qOCT}$ in relation to the optics of the system 
still needs further investigation. We hypothesize that the qAF/qOCT value with the master reference in the model eye will be able to serve as a compensation factor for in vivo applications, which also need further verification.

\subsection{2 in vivo simultaneous VIS-OCT and retina FAF imaging}

The system was tested for in vivo imaging on 3 groups of rats: 2-months-old albino, 14months-old albino, and 12-months-old pigmented rats. During imaging, the retinal OCT image was acquired with the shutter closed to block the light from the reference arm for the OCT reference target. The shutter was opened to image the reflectance reference target when the probe light was scanned to the corresponding location. AF images from the retinas of a young and an old albino rats were shown in Fig. 4-5(a) and 4-6(a), respectively. The intensities of the retinal AF images were normalized to that of the fluorescence reference target, the image of which is shown at the bottom right of each image. Since the OCT reference target is also fluorescent, although, off the peak detection range, it also showed up in the AF images (bottom left in each image). OCT fundus images (projection of the 3D OCT data on the X-Y plane or en face view) ${ }^{44}$ normalized to the reflectance reference target are shown in Fig. 4-5(b) and 4-6(b), respectively. Using manual segmentation on the OCT B-scans we calculated the reflectance projection of the RPE layer (Fig. 4-5(c) and 4-6(c)). For segmentation, several points on the RPE boundaries on several B-scans were manually selected and the boundary surfaces were estimated using interpolation. The average of the RPE reflectance was calculated after removing the optic disc and within a square window with a controlled distance from the optical disc. The window was selected within the region 
without the blood vessel shadows. This calculation is used later in comparison studies

(Fig. 4-7). Figures 4-5(d) and 4-6(d) show the OCT B-scans at the location marked with yellow dashed lines on the fundus projections.

a)

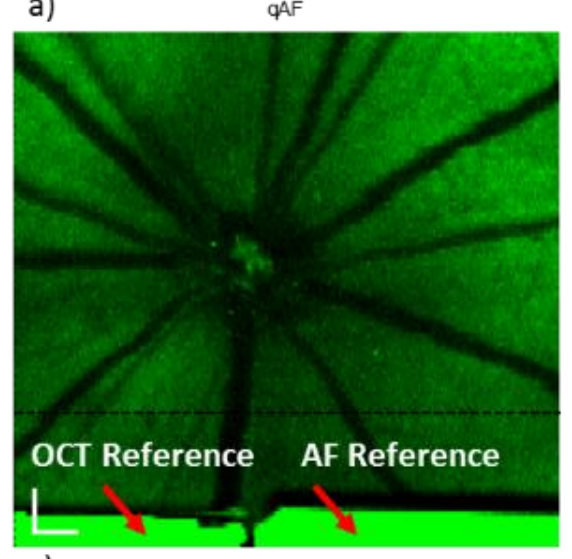

c)

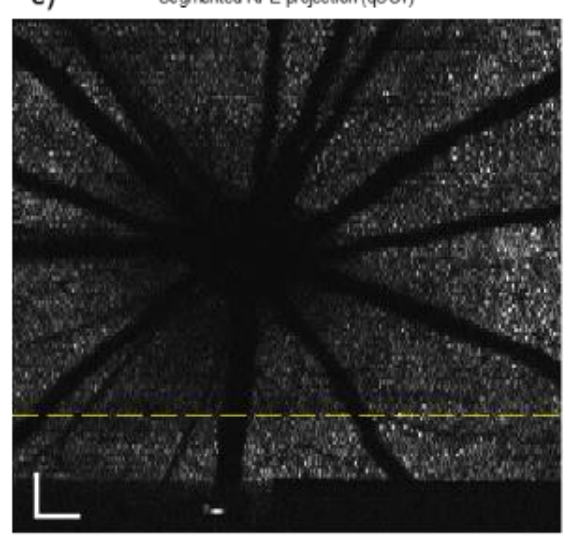

b)
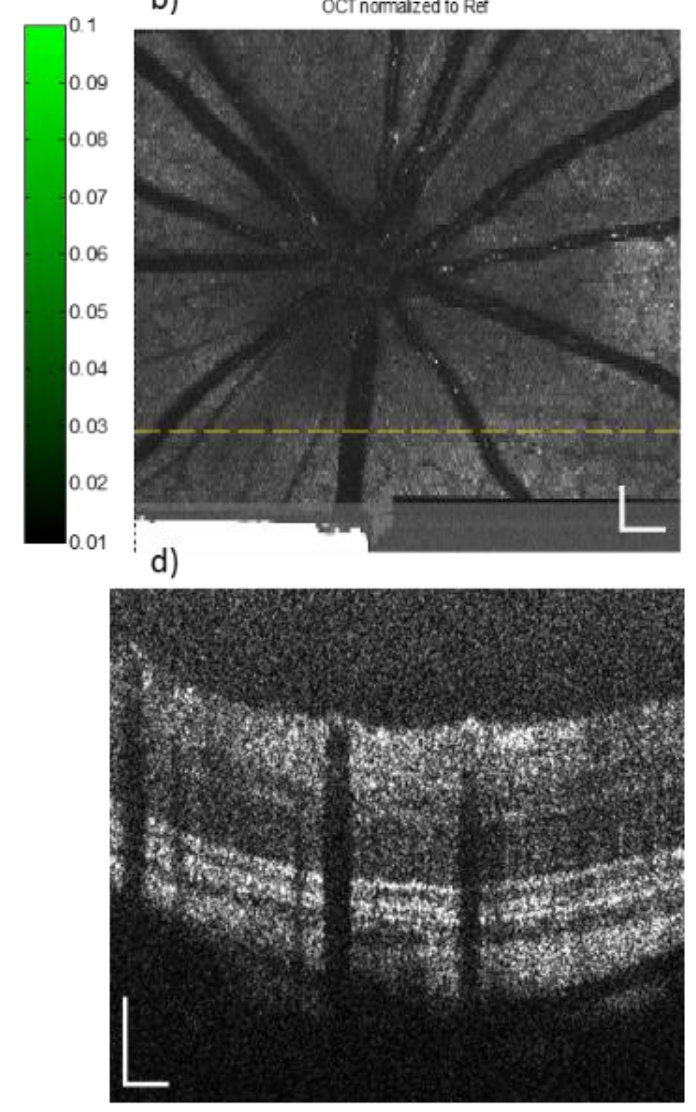

Figure 4-5 Simultaneous FAF and OCT images of a 2-months-old albino rat. a) AF image normalized to the fluorescence reference. b) Fundus OCT projection normalized to the reflectance reference. c) qOCT projection of the segmented RPE. d) OCT B-Scan at the location marked with a yellow dashed line. Bar: $200 \mu \mathrm{m}$. 
a)

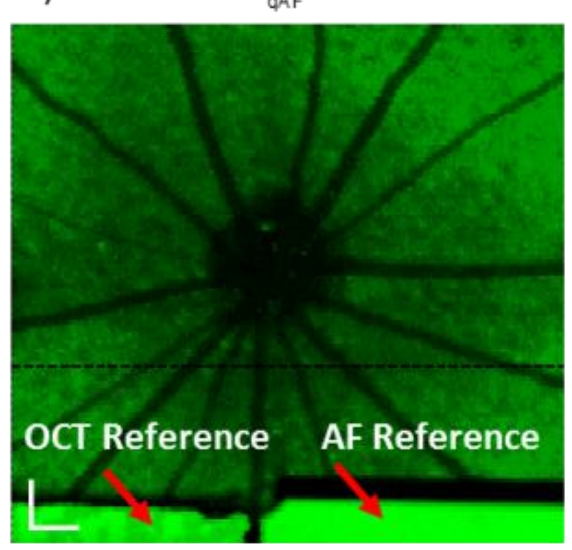

C) Segmented RPE projection normalzed

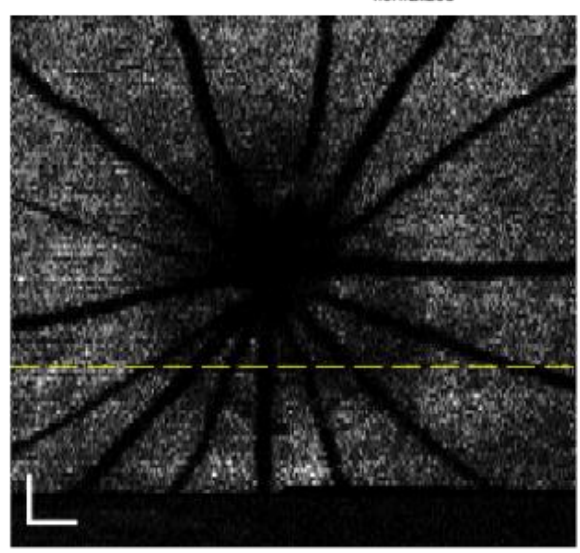

b)
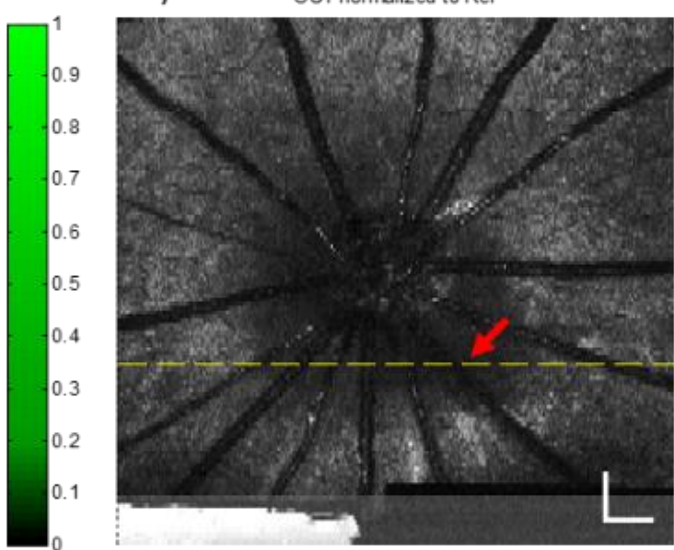

d)

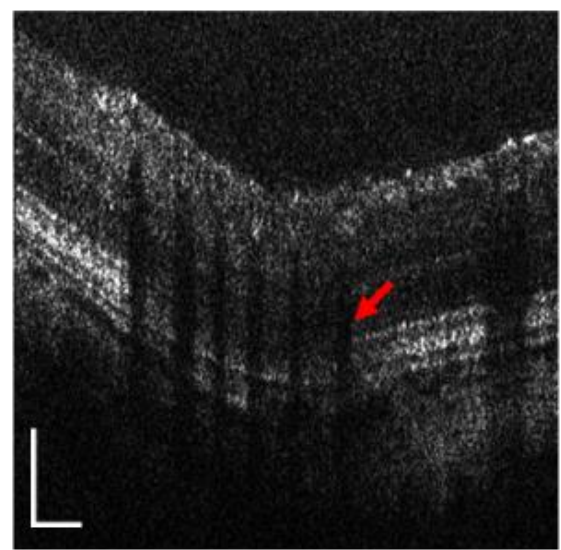

Figure 4-6 Simultaneous FAF and OCT images of a 14-months-old albino rat. a) AF image normalized to the fluorescence reference. b) Fundus OCT projection normalized to the reflectance reference. c) qOCT projection of the segmented RPE. d) OCT B-Scan at the location marked with a yellow dashed line. Bar: $200 \mu \mathrm{m}$.

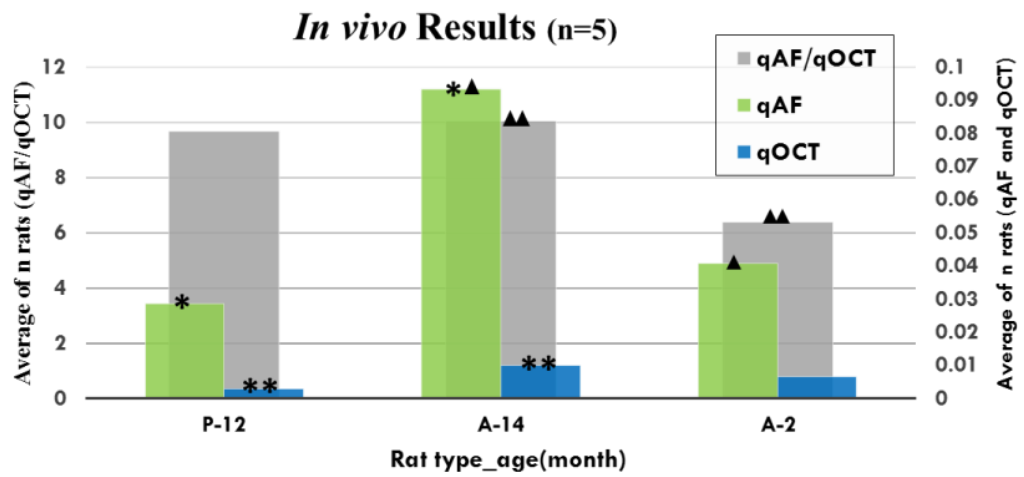

Figure 4-7 Comparison of the qAF, qOCT, and qAF/qOCT in three groups of albino and pigmented rats.

qAF was calculated by normalizing the averaged AF signal of the retina to the AF signal of the reference target. Similarly, qOCT was obtained by averaging the OCT reflectance 
signal of the RPE layer and normalizing it with that of the reflectance standard target. The ratio $\mathrm{qAF} / \mathrm{qOCT}$ was then calculated as a quantity only dependent on the optical properties of the RPE layer. The qAF/qOCT ratios were calculated for the images of the three groups of rats. As shown in Fig. 4-7, $\mathrm{qAF}(\mathrm{p}=0.0063)$ and $\mathrm{qAF} / \mathrm{qOCT}(\mathrm{p}=0.0325)$ levels in the older albino rats are significantly higher than the corresponding quantities in the younger albino rats, consistent with lipofuscin accumulation with aging ${ }^{31}$. On the other hand, the qAF level in the pigmented rats is lower than that of the 14-months old albino rats $(\mathrm{p}=0.0019)$, which agrees with previous studies ${ }^{84}$. However, the OCT reflectance signal of the segmented RPE layer in pigmented rats is also significantly lower than that of the 14-months old albino rats. As a result, the calculated qAF/qOCT ratio in the pigmented rats is not significantly different from the ratio in the albino rats $(\mathrm{p}=0.8021)$. Since melanin is distributed at a higher concentration in the apical portion of RPE cells, and lipofuscin is more concentrated in the basal portion ${ }^{87}$, melanin could significantly attenuate the $\mathrm{AF}$ signals. These results suggest that the $\mathrm{qAF} / \mathrm{qOCT}$ ratio is independent of melanin in the RPE, and thus the system is capable to compensate signal attenuation by RPE melanin. This feature of the system could have great clinical significance.

Acquired OCT fundus images from some of the old rats revealed noticeable dark regions around the optic disc (marked in Fig. 4-6(b)). The corresponding cross-sectional OCT images showed signs of photoreceptor loss or impairment (arrows in Fig. 4-5(d)). These findings may relate to the natural degeneration of the photoreceptors with age in albino rats, which were not further investigated in the current study. 


\subsection{Discussion}

We have demonstrated that the VIS-OCT based multimodal imaging technology is capable to obtain both OCT reflectance and AF images simultaneously. The intensities of the OCT reflectance from the RPE can serve as an internal reference to compensate signal attenuation by ocular tissues anterior to the RPE, including melanin in the RPE cells. The OCT and fluorescence signal intensities from the two reference standards placed in the intermediate retinal imaging plane can be used to not only eliminate the effects of light source fluctuation and detector sensitivity for OCT and AF imaging but also quantify the FAF signal to a value that is proportional to the absolute concentration of lipofuscin.

In our calculation, the calculated ratio, according to Eq. (8), is dependent on the RPE reflectance in addition to fluorescence efficiency. How to determine or measure the RPE reflectance needs further investigations. Weersink and co-workers ${ }^{104}$ studied the Fluorescence/Reflectance ratio using the diffuse reflection of the excitation light in a turbid media and used the ratio as a correction method to reduce the dependence of the fluorescence signal on tissue optical properties $\left(\mu \mathrm{a}, \mu \mathrm{s}^{\prime}\right)$. Their results showed a linear correlation between Fluorescence/Reflectance and fluorophore concentration. Although their results cannot be applied directly to our VIS-OCT and FAF imaging system, their experiments inspire us in the next step phantom studies.

In the current study, we used two separate reference targets for OCT and FAF because we used a high optical density ND filter to reduce the fluorescence signal of the reference target to the level of FAF. The high OD value of the ND filter (200 dB roundtrip attenuation) made it impossible for OCT to detect the reflectance from the reference target. 
In the future, a single customized standard reference target can be used for both FAF and OCT if the fluorophore concentration in the reference can be reduced so that the fluorescence signal from the target is in the level of FAF.

The constant values of qAF/qOCT in Fig. 4-3(c) and 4-3(d) do not agree with the prediction of Eq. (8), but the ratios agree with that of the fluorescent efficiencies of the two fluorescent dyes in the red (Texas Red) and green (FITC) slides. The results thus indicate that qAF/qOCT has a constant scaling factor for the values as predicted in Eq. (8). This constant scaling factor may be related to the geometric parameters of the model eye. Investigations with different parameters of the model eye could find a detailed interpretation of the scaling factor. It is important to determine this scaling factor for human eye imaging to optimize the system for the clinical application.

Our in vivo results show that the AF intensities from pigmented rat retinas, when normalized to the OCT reflectance as $\mathrm{qAF} / \mathrm{qOCT}$, are not significantly different from those in albino rats of similar ages. These results strongly suggest that our system is capable of compensating signal attenuation by melanin in the RPE cells. These findings could be of great clinical significance. Further investigations are warranted to correlate qAF/qOCT ratios of pigmented and albino animals at different ages to the actual lipofuscin levels from the same retinas determined chemically.

The accuracy of OCT reflectance from the RPE is important for determining the qAF/qOCT ratio. We manually segmented the OCT reflectance of the RPE layer in our in vivo experiments to provide proof-of-concept in the present work. Automatic segmentation 
by available software or new algorism could be used to improve the accuracy and to avoid bias and human error ${ }^{105}$.

The assumption that the same attenuation factors apply to excitation and emission wavelengths was based on the analysis of the total transmission of the ocular media. The total transmission of the ocular media was reported by Boettner and Wolter which includes the loss due to the normal incident light reflection at the tissue boundaries, scattering, and absorption. We have illustrated this attenuation effect by $\tau_{\text {pre }}\left(1-\rho_{\text {pre }}\right)$ in Eq. 6 and 7 . The total transmittance of ocular media is relatively constant from $450 \mathrm{~nm}$ to $600 \mathrm{~nm}$ with less than $10 \%$ increase with wavelength ${ }^{15}$.

\subsection{Conclusion}

We have developed a new multimodal imaging system with two reference targets placed in the intermediate retinal imaging plane for quantitative imaging of RPE lipofuscin. The system employs a single light source to acquire AF and VIS-OCT images of the retina simultaneously. Since the same group of photons is responsible for both AF and OCT imaging, the OCT image intensities can be used to compensate signal attenuation by media anterior to the RPE, including melanin in RPE cells. The reference standards are used not only to eliminate the influence of fluctuation in illumination power and in detector sensitivity but also to calculate the qAF to qOCT ratio of the RPE layer. This work is a major step towards determining the absolute AF intensity and correlating it to the lipofuscin content in the RPE. 


\section{CHAPTER 5 A2E reference target}

\subsection{Introduction}

Lipofuscin is the hallmark of aging and some retinal disease like Age-related Macular Degeneration (AMD) ${ }^{106,107}$, Stargardt's, and Best ${ }^{97,108-110}$ that accumulates in Retinal Pigment Epithelium (RPE) and eventually can lead to irreversible loss of vision. Lipofuscin is a non-degradable mixture of lipids, proteins, and small chromophores, partly from phagocytosis of photoreceptor outer segments that shed into the RPE. It is also the major source of fundus autofluorescence (FAF). N-retinylidene-N-retinylethanolamine (A2E) is the major hydrophobic fluorescent component of lipofuscin that forms from the reaction of two molecules of all-trans retinal with ethanolamine that are both molecular compounds of Photoreceptor outer segments. Studies indicate the involvement of A2E in the process of AMD and potential target molecule for treatment. ${ }^{111}$

The identification of A2E in the lipofuscin granules led to a variety of studies to understand the photochemistry of A2E and its role in phototoxicity of lipofuscin ${ }^{112}$. The role of A2E is still in debate; preliminary assays suggested $\mathrm{A} 2 \mathrm{E}$ as a leading factor in the formation of lipofuscin ${ }^{113}$, and accumulation of $\mathrm{A} 2 \mathrm{E}$ in the human eye might be responsible for the formation of RPE debris ${ }^{114}$. Evidently irregular production of this toxic byproduct of the visual cycle can lead to rapid and progressive retinal degeneration ${ }^{115}$. Other studies focused on the phototoxicity of A2E and A2E oxides on human RPE ${ }^{116}$, which results in blue light-induced retinal injury ${ }^{117}$. In recent years synthesizing A2E has drawn scientists' interest in studying the mode of interactions in RPE cells, RPE enzymes, phototoxicity, 
and metabolic changes of A2E molecule itself ${ }^{118}$. Various methods have been developed and investigated for A2E synthetization. Basically, A2E can be synthesized by combining all-trans-retinal and ethanolamine (2:1), followed by gravity chromatography separation and reverse-phase High-Performance Liquid Chromatography (HPLC) ${ }^{119}$ or liquid partitioning ${ }^{120}$. Incubator and microwave synthesizer have been used to scale up the process ${ }^{121}$. Optimization of the in vitro synthesis of A2E resulted in the one-step biomimetic preparation of this pigment and consecutively quantification and characterization of A2E and its isomers ${ }^{122}$. Administration of synthesized A2E and its oxiranes ${ }^{123}$, revealed the detergent-like effect of this compound on the membrane that depends on A2E concentration ${ }^{124}$ and it can be a primary driver of retinal degeneration ${ }^{120}$. Therefore, prevention or reduction of this accumulation is a cautious strategy to prevent blindness ${ }^{11}$. Polarographic analysis ${ }^{119}$ and cyclic voltammetry ${ }^{125}$ of synthesized A2E has been used to determine the reduction potential of A2E.

While in vitro studies on $\mathrm{A} 2 \mathrm{E}$ characterization and quantification can lead to the discovery of treatment methods to prevent irreversible blindness, in vivo quantification of A2E or lipofuscin granules is essential in the diagnosis of retinal degeneration in early stages 35,37,126. For example, AMD starts with the early accumulation of deposits in and between RPE and Burch Membrane (BM) and atrophy of inner and outer segments happen in end stages. Fluorescent emission of lipofuscin components, specifically A2E has led to the invention of FAF imaging systems. However, quantification of lipofuscin/A2E requires measurement of true values of FAF that are independent of acquisition system parameters and optical properties of ocular media in front of lipofuscin which varies between people and for individuals over time. In 2011 Delori implemented a fluorescent reference target 
into scanning laser ophthalmoscope (SLO), an HRA2 and an S3300 Spectralis HRA-OCT (both Heidelberg Engineering, Heidelberg, Germany), and introduced quantified autofluorescent (qAF) that is independent of acquisition parameters such as detector sensitivity ${ }^{16}$. In 2016 we ${ }^{31}$ took advantage of simultaneous visible light OCT and FAF to compensate for attenuation caused by ocular media prior to RPE and later in 2018 achieved quantitative imaging by implementing Delori's reference target to the VIS-OCT-FAF system ${ }^{33}$. Although the new technology compensated for both ocular attenuation and system differences, quantification of absolute concentration of lipofuscin is yet to be achieved. One major limitation was the significant difference between the quantum yield, absorption, and fluorescence spectrum of lipofuscin/A2E and commercialized reference target (Texas Red). Moreover, the fluorescent efficiency of the Texas red target is significantly higher than that of the FAF which necessitated the use of a neutral density (ND) filter (optical density $(\mathrm{OD})=10$ ) and consecutively a second target for OCT quantification.

In this chapter, we introduce a new customized reference target for FAF imaging consisting of synthesized A2E, the major fluorophore of FAF. A2E was synthesized by a chromatography-free method to prepare gram quantities of this vitamin A dimer. Polymethyl-methacrylate (PMMA) was used as the substrate for the fabrication of the customized target. Similar fluorescent characteristics of the new target to FAF provides a better tool for standardization of FAF imaging. With known and controlled concentration of synthesized A2E, a single reference target was implemented into the VIS-OCT-FAF system for the quantification of both VIS-OCT and FAF. We successfully performed in vivo imaging of rat retina and fluorescence efficiency of both FAF and A2E references 
were in the linear detection range and eliminated the use of ND filter. in vivo quantification of the absolute concentration of retina Lipofuscin/A2E with reference to the A2E target is presented in the next chapter.

\subsection{Methods}

\subsubsection{A2E synthetization}

A2E was synthesized following a published method by Penn et al. ${ }^{120}$. Ethanol $(31.3 \mathrm{ml})$, ethanolamine $(3.8 \mathrm{ml})$, acetic acid $(4.5 \mathrm{ml})$, and all-trans-retinal $(1 \mathrm{~g})$ were added to a 50$\mathrm{ml}$ tube and allowed to react at room temperature in the dark for 2 days with gentle rocking. When the reaction was complete, the ethanol was evaporated. The reaction mixture was dissolved in acetonitrile, washed 5 times with hexane and $1 \mathrm{M}$ sodium acetate (1:1). The middle layer was collected after each wash. The reaction product was washed one more time with $\mathrm{H}_{2} \mathrm{O}$ and dried in a SpeedVac (Savant Instruments) overnight. Synthesized A2E was stored under argon at $-20^{\circ} \mathrm{C}$ in the dark.

\subsubsection{A2E-PMMA fabrication}

We chose PMMA-resist for the target substance. PMMA is widely used in optical components for its high level of clarity and its rigid structure ${ }^{127}$. PMMA is most commonly used as a high-resolution resist for many imaging and non-imaging applications ${ }^{128,129}$. PMMA resist can be easily prepared by solving PMMA powder in various solvents with a lower polarity such as chlorobenzene, toluene, acetone, anisole, over hotplate $\left(50^{\circ} \mathrm{C}\right)$. Here

we used the safe and recommended solvent ${ }^{130}$, anisole, which is also a suitable solvent for A2E due to its polarity. 
We were successfully able to obtain a homogenous solution of PMMA and A2E in anisole, however, to minimize the human error, and to have a standardized reference for customized target characterization, final target was fabricated with Nano PMMA resist with molecular weight (MW) of 950k (MicroChem A4, solvent: anisole, 4\%). $24 \mathrm{mg}$ of synthesized A2E was then mixed with $20 \mathrm{ml}$ of PMMA resist to obtain $30 \mathrm{~g} / \mathrm{l}$ concentration in the cured solid PMMA slide. The optimum concentration was empirically chosen by measuring the fluorescent efficiency of different concentrations by VIS-OCT-FAF ${ }^{33}$ and comparing the values with that of in vivo rodent retina.

The solution was stirred for 3 days in a closed container in dark to ensure the complete dissolution of A2E particles while minimizing photobleaching of A2E. The homogeneity of the solution was confirmed by coating a thin layer (Theoretical thickness $=400 \mathrm{~nm}$ with spinning speed of $1000 \mathrm{rpm}^{129}$ ) of the resist on a silicon wafer and curing on the hot plate. Images from VIS-OCT-FAF did not show any undissolved particle (Fig. 5-2(a and b)). Different dissolving methods such as dissolving A2E in ethanol and methanol prior to adding to the PMMA resist and filtering the solution by a 20-micron syringe filter were tried. However, coatings on silicon wafers showed better homogeneity by increasing the stirring time to 3 days. 


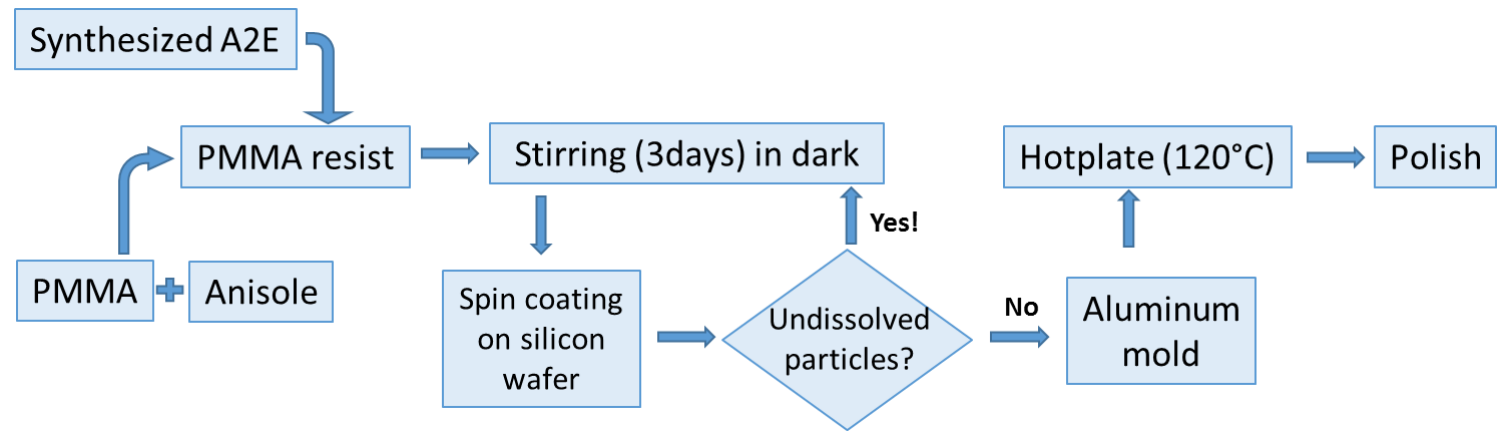

Figure 5-1 Fabrication process of the A2E reference target

(a)

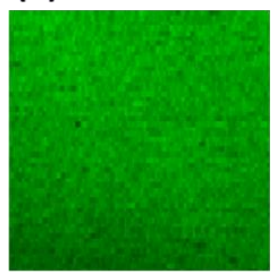

(b)

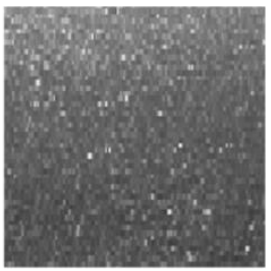

(c)

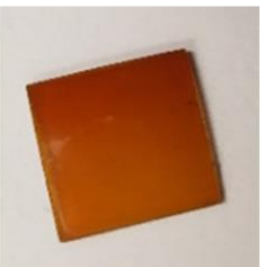

(d)

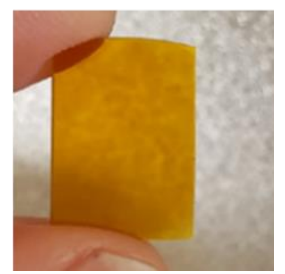

Figure 5-2 PMMA-A2E homogenity test and final A2E target, a) Homogeneous AF image of the PMMAA2E coated on silicon wafer, b) OCT image of the coated wafer, c) A2E-PMMA cured and cut into squareshaped peice, d) A2E PMMA taregt after polishing.

To fabricate the PMMA-A2E reference target, we tried several methods including spin coating the resist on a silicon wafer, Deep Reactive Ion Etching (DRIE) to create small molds for PMMA resist, and finally, using off the shelf aluminum mold for casting. Due to the high complexity of the first two methods and greater affordability and availability of the third method, the later was chosen for fabrication.

We poured the $40 \mathrm{ml}$ PMMA-A2E in a cupcake aluminum mold. The aluminum container was filled with the resist and covered by an aluminum sheet to avoid surface evaporation and achieve a flat surface. The PMMA-A2E resist was cured on the hot plate at 120C for 4 hours. The circular plate was then cut using $\mathrm{CO} 2$ laser engraving to make multiple squareshaped reference targets (Fig. 5-2(c)). The rigid A2E-PMMA target was then polished with 3000,5000 , and 7000 assorted high grits polishing sandpaper, respectively, to ensure the 
uniformity of thickness and roughness over the surface of the target. Then the target was polished by a wet and soft cloth with first, plastic fine scratch remover (NOVUS2) and then, plastic cleaner and shiner (NOVUS PC-10) to achieve optically flat surface (Fig. 52(d)).

\subsubsection{Reference Characterization}

We characterized the reference by measuring its reflectance and fluorescence properties. The reflectance of the polished target was calculated by using a non-polarizing beam splitter (NBS) and power meter as demonstrated in figure 5-4(a). The reflectance was calculated by finding the ratio of the reflected light power to the illumination power in front of the sample. In this calculation, we have measured and considered the loss of light in the beam splitter.

Absorption and emission spectrums were measured by VIS-UV spectrophotometer (Varian Cary) and a house-built fluorospectrometer (Fig. 5-3(b)), respectively. The spectrofluorometer is based on epi-illumination (illumination and observed light are both on the same side of the specimen) utilizing an objective lens. We used a compact laser diode (CLD 1011LP, Thorlabs) with a central wavelength of 488nm suitable for lipofuscin/A2E excitation. Blue light passes through fiber to collimator where collimated light is projected to a dichroic mirror (DMLP505 - Ø1" Long pass, $505 \mathrm{~nm}$ Cutoff, THORLABS) in a 45degree angle and reflected onto the sample with a microscope objective lens (Newport). Fluorescence emission is collected by an objective lens and passes through the dichroic mirror (cut-on: $505 \mathrm{~nm})$ and then a notch filter $(488 \mathrm{~nm})$ to eliminate the excitation light. Fluorescence light is then focused by focusing lens $(\mathrm{f}=30 \mathrm{~mm})$ and detected by the detector 
of the spectrometer (OceanOptics USB4000). The SpectaSuite software is used to further analyze the fluorescence spectrum. ${ }^{131}$

(a)

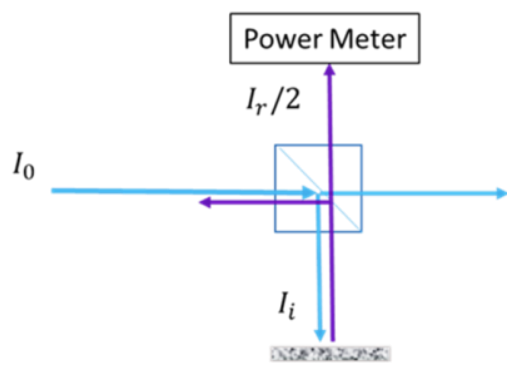

(b)

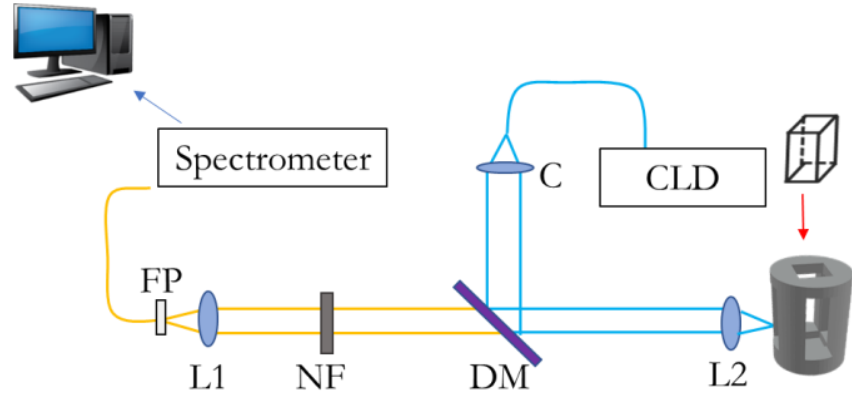

Figure 5-3, Characterization methods of A2E reference: a) Reflection measurement, b) Spectrofluorometer schematic

\subsection{Results}

\subsubsection{Reference Target}

The reflectance of the reference target was measured to be 0.04 , consistent with the theoretical specular reflectance of PMMA with a refractive index of 1.49 using theoretical equation $R=\left(\frac{n_{1}-n_{2}}{n_{1}+n_{2}}\right)^{2} \cong 0.04$, where $\mathrm{n}_{1}$ and $\mathrm{n}_{2}$ are the refractive indexes of air and PDMS.

\subsubsection{Fluorescence}

According to the findings by Jin et al ${ }^{132}$, A2E is are stable in dimethyl sulfoxide (DMSO) and methanol. Therefore, the results were compared with the absorption and emission spectrum of A2E in methanol. Fluorescence emission of PMMA-A2E is illustrated in figure 5-4(a) which is in agreement with A2E in methanol (fig. 5-4(b)). Therefore, the characteristics of A2E has not been altered. We also measured emission spectrums of 4 
samples of human lipofuscin, the results show a close approximation to the synthesized A2E spectrum (fig 5-4 (c)).
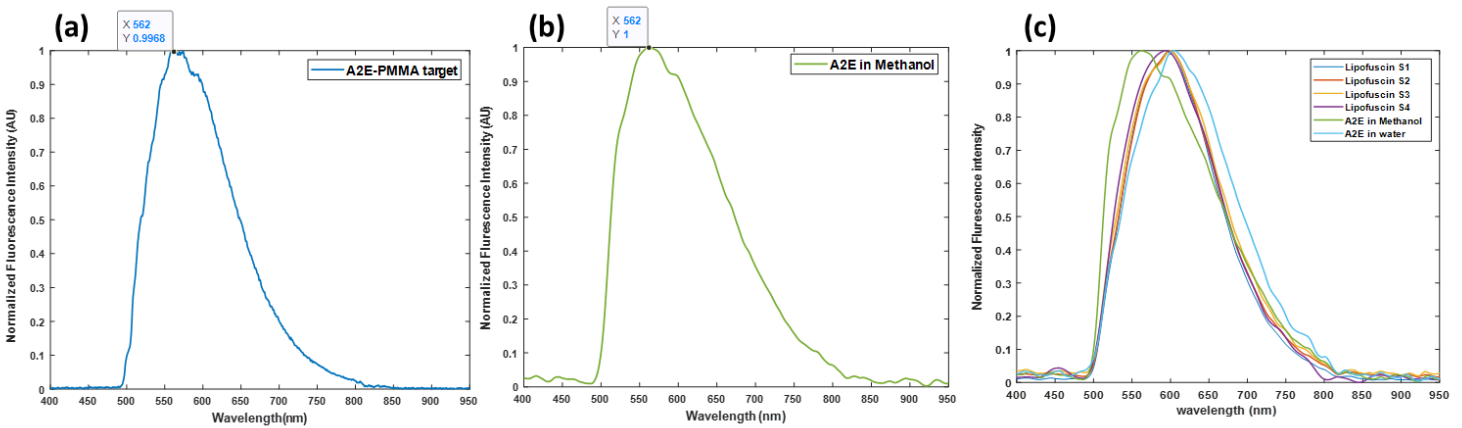

Figure 5-4 Fluorescence emission spectrums of A2E and lipofuscin: a) PMMA-A2E target, b) A2E in methanol, c) Human lipofuscin compared to synthesized A2E

Quantum yield of the A2E solutions and the customized target was measured with respect to a known reference to rhodamine B and fluorescein. Several solutions with increasing fluorophore concentration were prepared for standard and test fluorophores. Fluorescence intensity and absorption spectrum of these solutions were recorded with spectrofluorometer and spectrophotometer, respectively. The integrated fluorescence intensity vs absorption at the excitation wavelength $(488 \mathrm{~nm})$ is plotted and the gradient of the linear regression will be used to calculate the quantum yield of the test fluorophore ${ }^{133}$ from the following equation.

$$
\phi_{X}=\phi_{S T}\left(\frac{\operatorname{Grad}_{X}}{\operatorname{Grad}_{S T}}\right)\left(\frac{\eta_{X}^{2}}{\eta_{S T}^{2}}\right)
$$

where $\mathrm{X}$ and ST denote test and standard fluorophores and $\phi, \eta$, and Grad are the fluorescent quantum yield, refractive index, and gradient of the plot, respectively. 
In this study fluorescein and rhodamine B were used as standard fluorophores because of their availability and similar emission spectrum to A2E. We confirmed absorption measurements by linear correlation of absorption with concentration and calculating the extinction coefficients of fluorescein and Rhodamine B at 488nm illumination. ${ }^{134,135}$ The standard samples were then first cross-calibrated and then separately used for calculation of A2E quantum yield. We found an extinction coefficient of $3127.8 \mathrm{M}^{-1} \mathrm{~cm}^{-1}$ for synthesized A2E while concentrations were calculated based on the reported A2E molecular weight of $592(\mathrm{~g} / \mathrm{mol}) .^{136}$

Figure 5-5 illustrates the results of the integrated emission spectrum versus absorption values. The gradient of this plot was used in Eq (1) for quantum yield calculation. CrossCalibrating fluorescein and rhodamine B resulted in quantum yields of 0.92 and 0.65 , respectively, which is consistent with reported $\phi$ values 0.925 for fluorescein ${ }^{137}$ and 0.65 for rhodamine $\mathrm{B}{ }^{133}$. A2E quantum yield with reference to Fluorescein and Rhodamine B was calculated $0.003 \pm 0.001$, respectively, which is comparable to the low emission quantum yield of A2E in methanol in previous investigations. ${ }^{138,139}$
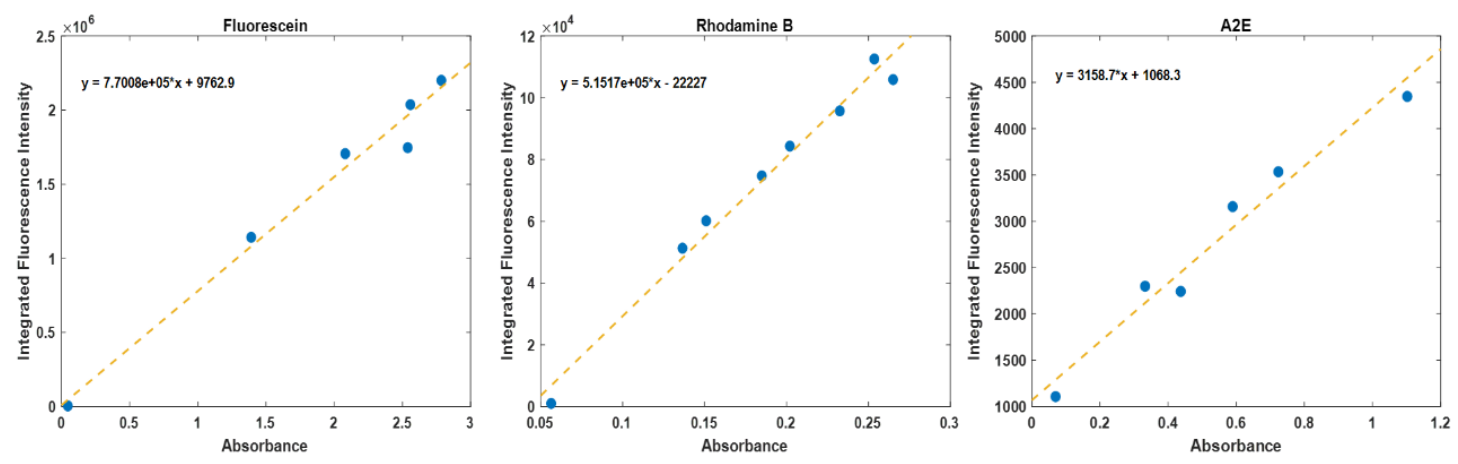
Figure 5-5 Integrated fluorescence vs. Absorbance: a) Rhodamine B, b) Fluorescein, c) Synthesized A2E

\subsubsection{In vivo Experiment}

To assess the capability of VIS-OCT-FAF with A2E reference target in the quantification of qAF, qOCT, and RPE lipofuscin, the reference target was implemented in the retinal intermediate imaging plane and covered about $13 \%$ of the bottom of the field of view.

we imaged a 9-month-old Long Evan rat (Fig. 5-6). The Experiments were conducted in agreement with the ARVO Statement for the Use of Animals in Ophthalmic and Vision Research and with the guidelines of the Florida International University's Institutional Animal Care and Use Committee.

A cocktail of Ketamine (54mg/kg body weight) and Xylazine ( $6 \mathrm{mg} / \mathrm{kg}$ body weight) was Intraperitoneally injected for animal anesthetization. A hard contact lens was put on the animal eye to prevent corneal dehydration and cataract formation. The rat was restrained in an animal mount with five degrees of freedom.

Figure 5-6(a) is the en face fundus projection of VIS-OCT found by the summation of the signals along the A-lines. Figure 5-6(b) shows the FAF normalized to the AF of the A2E reference target and figure 5-6(c) shows the OCT cross-sectional image of the retina at the location marked on the top of the figure 5-6(a and b). Finally, figure 5-6(d) is the crosssectional image of the A2E reference target marked on the bottom of figure 5-6(b).

In this setup A2E reference has been used without an absorptive filter and the AF values are comparable with those of FAF. Elimination of the ND filter made this target also usable for OCT quantification. 
(a)

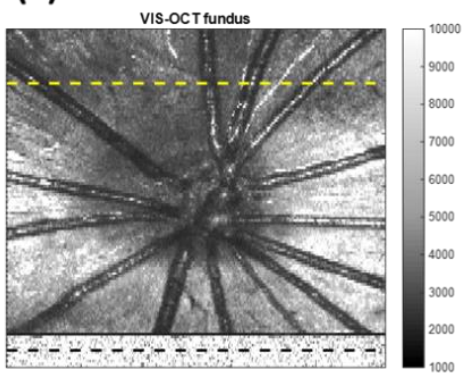

(c)

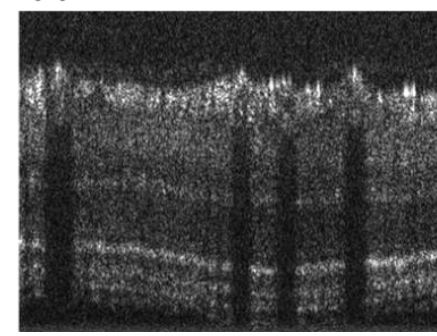

(b)

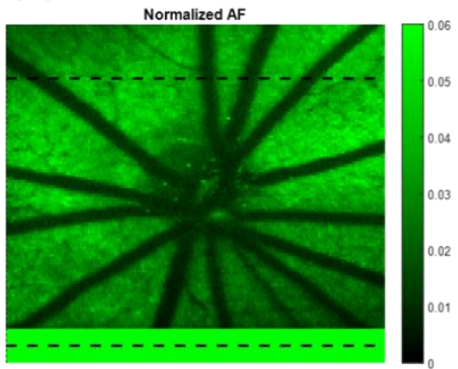

(d)

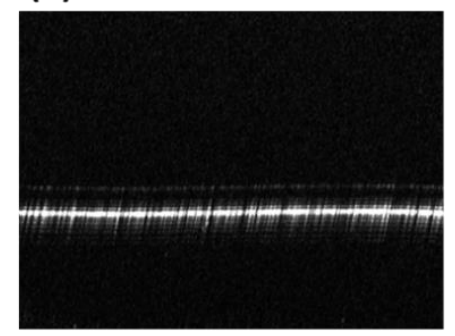

Figure 5-6 Simultaneous in vivo VIS-OCT and FAF of a 9-month-old pigmented rat a) en face OCT fundus projection, b) FAF normalized to A2E reference target, c) OCT B-scan of the location marked on top of (a and b), d) OCT B-scan of the A2E reference target marked on the bottom of (a \& b)

\subsection{Conclusion}

We successfully fabricated and characterized a single customized reference target for both VIS-OCT and FAF with synthesized A2E. Similar fluorescence characteristic and efficiency of A2E reference target to FAF makes it suitable for in vivo quantification of RPE lipofuscin. This reference will be for quantitative studies with VIS-OCT-FAF to provide a diagnostic tool for retinal degenerative diseases that are integrated with lipofuscin accumulation in RPE cells. 


\section{CHAPTER 6 RPE Simulating Phantoms for VIS-OCT-FAF}

\subsection{Introduction}

Optical Coherence Tomography (OCT) and Fundus Autofluorescence imaging are two important modalities in ophthalmic diagnostic and monitoring. However, there has been relatively little test methods to assess the performance of these modalities. ${ }^{140}$ To ensure quality control over time and provide a consistent measurement for comparison, wellvalidated physical models called phantoms are used. Retinal simulating phantoms have been fabricated and used for several applications such as surgery simulation, imaging, and quantitative studies ${ }^{141}$. Nevertheless, the assessment of optical imaging technologies requires the simulation of optical characteristics of retinal layers ${ }^{142-144}$.

Development of simultaneous visible light OCT and FAF (VIS-OCT-FAF) has shown promising results in the quantification of retinal lipofuscin, a biomarker for retinal degenerative disease. Recent findings suggest that VIS-OCT-FAF can quantify the fluorophore content of retinal pigment epithelium (RPE), lipofuscin, independent of attenuation caused by pre-RPE media as well as RPE melanin. Melanin granules are abundant in adult human RPE and predominantly located in the apical and mid-portion 143,146. Several studies have identified the protective role of melanin in the absorption of scattered light in the RPE ${ }^{147}$. It is known that upon absorption of light melanin granules move to the apical region of the RPE microvilli which surround the outer segments of photoreceptor cells. Light-driven translocation of RPE melanin granules (melanosomes) has been observed in frogs ${ }^{148,149}$ and other animals ${ }^{150,151}$. Further comparison OCT studies 
between albino and pigmented retinas, and between light-adapted and dark-adapted retinas show that light-driven pigmentation increases the reflectance of the photoreceptor layer while decreasing the OCT intensity of the RPE layer ${ }^{149,152}$. Similarly, melanin can attenuate the FAF signal by absorbing both excitation and emission lights originated from lipofuscin which is mainly located in the basal portion of RPE cells ${ }^{35,87}$.

Pigmentation concentration might vary between people; for example, lack of RPE melanin has been observed in albinos ${ }^{152}$ and melanosomes distribution can be altered in time or in certain pathological disorders ${ }^{153}$. Although the attenuation of both OCT and FAF signals by RPE pigmentation, has been observed in vivo ${ }^{154-156}$, it has not been quantitatively investigated in well-validated phantoms with controlled optical properties. Current retinal simulation phantoms for OCT simulate the optical properties of the retinal layers in NIR range ${ }^{140,149}$ and do not exhibit fluorescence properties.

In this study, we fabricated RPE simulating phantoms with similar optical properties of the PRE layer in the visible spectrum to assess the VIS-OCT-FAF system in compensating RPE melanin attenuation. The phantoms were fabricated with similar thickness, fluorescence, scattering and absorption properties similar to the RPE layer when illuminated with 480nm, the wavelength of VIS-OCT-FAF light source.

We measured the absorption and scattering of the RPE simulating phantoms with integrating sphere and Inverse Adding and Doubling (IAD) method for optimum concentration of scatterers and absorbers. Fluorescent phantoms with varying absorbers concentrations were then fabricated and imaged with VIS-OCT-FAF. The technology significantly compensated for the attenuation caused by simulated RPE melanin. 


\subsubsection{Phantom fabrication}

\subsubsection{Simulating RPE optical properties}

The thickness of the RPE layer in the human retina has been reported to be between 10-30 $\mu \mathrm{m}$ with an average absorption coefficient $\left(\mu_{\mathrm{a}}\right)$ of $110-115 \mathrm{~mm}^{-1}$ and a scattering coefficient of $130 \mathrm{~mm}^{-1}$ with approximately 0.84 anisotropy factor $(\mathrm{g})$ at $488 \mathrm{~nm}$ wavelength ${ }^{142}$. Lipofuscin/A2E is the major fluorophore of the RPE layer and its excitation and emission spectrums have been previously investigated ${ }^{98}$. The fluorescence emission spectrum of A2E has a peak of around $600 \mathrm{~nm}$ and can be excited most efficiently around $430 \mathrm{~nm}^{157}$.

To fabricate thin RPE simulating layers, we Polydimethylsiloxane (PDMS) as the substrate for its durability and clear structure and used spin coating to achieve micrometer thickness. According to the previous studies, $\mathrm{TiO} 2$ is the most efficient scatterer to simulate high scattering properties of RPE ${ }^{140}$. Carbon is also commonly used for simulating optical attenuation of tissue melanin ${ }^{158,159}$. In this study, $\mathrm{TiO} 2$ and Carbon particles were used to simulate scattering and absorption coefficients of RPE, respectively. We used Rhodamine $\mathrm{B}$ as the fluorophore due to its high solubility and quantum yield. Besides, A2E/lipofuscin and Rhodamine $\mathrm{B}$ share the same range of wavelengths in extinction and emission spectrums. ${ }^{160}$

We mixed the increasing concentrations of $\mathrm{TiO} 2$ and Carbon particles with PDMS (SYLGARD ${ }^{\mathrm{TM}} 184$ Silicone Elastomer Kit, DOW), stirred and sonicated for one hour consecutively for a total of 10 hours. Then curing agent (Sylgard) was added to the homogenous suspension (1:10), stirred for 10 minutes, and degassed under a vacuum chamber for 30 minutes. In order to estimate the range of particle concentrations for 
phantoms with a thickness of 10-30 $\mu \mathrm{m}$ and RPE absorption and scattering coefficients, we first fabricated a set of $2 \mathrm{~mm}$-thick phantoms, cured in aluminum molds in an oven (300 F) for 10 minutes, with small and increasing concentrations of $\mathrm{TiO} 2$ and carbon, separately. We measured the absorption spectrums of carbon phantoms with a VIS-UV spectrophotometer (Varian Cary) and calculated the extinction coefficient of carbon in PDMS to estimate the required carbon concentration and achieve the desired absorption coefficient $\left(\mu_{\mathrm{a}}=110-115 \mathrm{~mm}^{-1}\right)$ within a $10-30 \mu \mathrm{m}$ thick phantoms. An integrating sphere with the IAD method was used to measure the scattering coefficient of $\mathrm{TiO} 2$ phantoms. Based on the linear correlation of measured $\mu_{\text {s }}$ with $\mathrm{TiO} 2$ concentration, the required concentration was then estimated to achieve $\mu_{\mathrm{s}}=130 \mathrm{~mm}^{-1}$ in $10-30 \mu \mathrm{m}$ thick phantoms.

Micron-thick phantoms were then fabricated spin coating the PDMS suspension on glass slides with $2000 \mathrm{rpm}$ speed for 1 minute. The phantoms were then cured on a hotplate for 3 minutes $(300 \mathrm{~F})$. The optimum concentration of the scatterers and absorbers were selected imperially by measuring absorption and reduced scattering coefficients of glass-coated phantoms with an integrating sphere and IAD method. In these calculations, we assumed: $\mathrm{g}=0$. The thickness of the phantoms was measured to $30 \pm 2 \mu \mathrm{m}$ with both profilometer and micron digital thickness gauge.

\subsubsection{Simulating melanin-induced attenuation in fluorescent phantoms}

To simulate the attenuation role of melanin we fabricated a set of two-layered phantoms:

first, a fluorescent scattering layer that simulates the lower and middle part of RPE containing lipofuscin granules and second, an absorptive layer to simulate apical surface containing melanin granules. Using a similar method explained in the previous section, the 
lower layer was fabricated with PDMS, TiO2, and increasing concentration of Rhodamine B. The suspension was coated on a glass slide and cured before adding the second layer. The top layer contains PDMS and an increasing concentration of carbon particles for each previously coated Rhodamine B concentration. In other words, a set of 2-layered phantoms was fabricated with a $2 \mathrm{D}$ matrix of carbon and rhodamine $\mathrm{B}$ concentrations. We investigated the role of RPE melanin in attenuating FAF and OCT signals and assessed the linearity of qAF and qAF/qOCT values with respect to fluorophore concentration.

\subsection{Results}

\subsubsection{Simulating RPE optical properties}

We measured with the transmittance and reflectance of two sets of $2 \mathrm{~mm}$ thick phantoms, one with increasing concentration of $\mathrm{TiO} 2$, and others with increasing concentration of Carbon. Figure (6-1(a)) shows the fabricated phantoms. Figure (6-1(b)) illustrates measured absorption by spectrophotometer for different concentrations of carbon and figure (6-1(c)) shows the reduced scattering coefficients measured by an integrating sphere for different concentrations of $\mathrm{TiO} 2$. Based on the linear correlations, we estimated that the proper range of concentration for both particles is within 1-10\% of the PDMS weight $(\mathrm{wt} \%)$. 
(a)

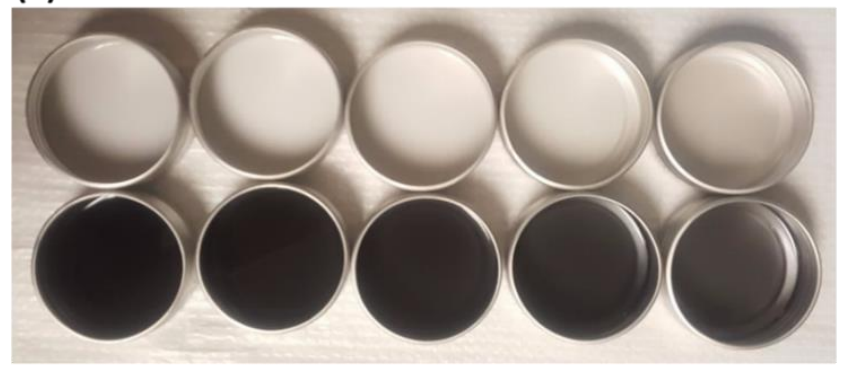

(b)

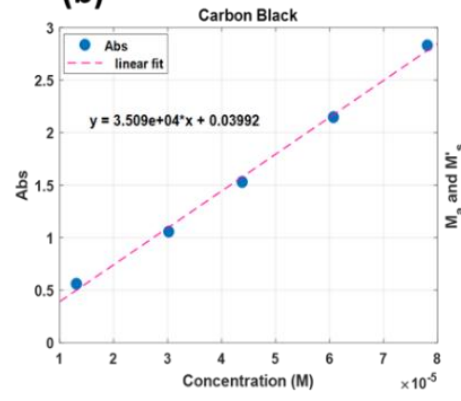

(c)

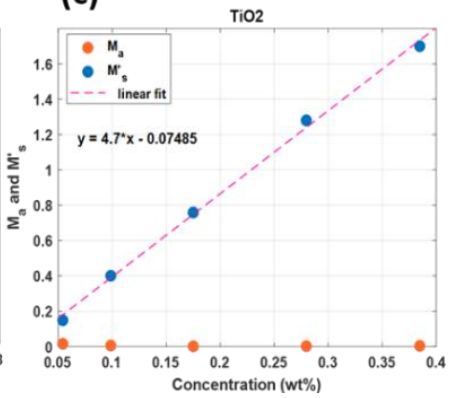

Figure 6-1 Evaluation of PDMS-TiO2 and PDMS-Carbon phantoms: a) 2mm-thick PDMS phantoms with increasing $\mathrm{TiO} 2$ and Carbon concentrations measured by spectrophotometer. b) Absorbance versus concentration of PDMS-Carbon Phantoms. c) Absorption and reduced scattering coefficients of PDMS-TiO2 phantoms measured by an inegrating sphere.

Reduced scattering coefficients of $30 \mu \mathrm{m}$-thick phantoms were measured for a $1-10 \%$ concentration of $\mathrm{TiO} 2$ particles. Figure 6-2(b) illustrates the linear increase of qOCT and $\mu$ 's with $\mathrm{TiO} 2$ concentration. Assuming an anisotropy factor of zero for PDMS-TiO2 $\left(\mathrm{g}_{\mathrm{PDMS}-\mathrm{TiO} 2}=0\right), 6 \mathrm{wt} \%$ concentration of $\mathrm{TiO} 2$ is optimum to achieve $\mu$ 's $=20\left(\mathrm{~g}_{\mathrm{RPE}}=0.84\right)$.

With a constant concentration of $\mathrm{TiO} 2(6 \mathrm{wt} \%)$, we fabricated a second set of phantoms with

increasing carbon concentrations $(0.5,1,2,3 \mathrm{wt} \%)$. Figure (6-2) illustrates the PDMS-TiO2Carbon phantoms (6-3(a)) and measured absorption coefficients (6-3(c)). Considering the additional absorption that will be caused by introducing the fluorophore (Rhodamine B), 
4wt\% and lower concentration of carbon can result in achieving the RPE absorption coefficient.

(a)

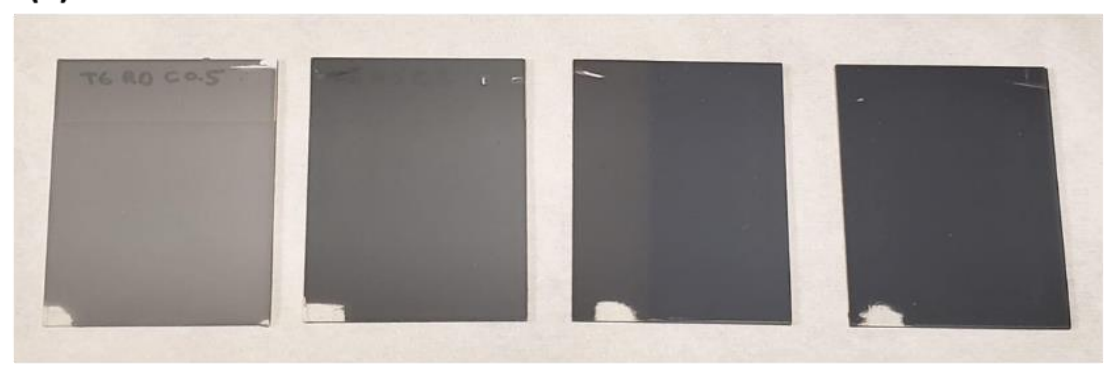

(b)
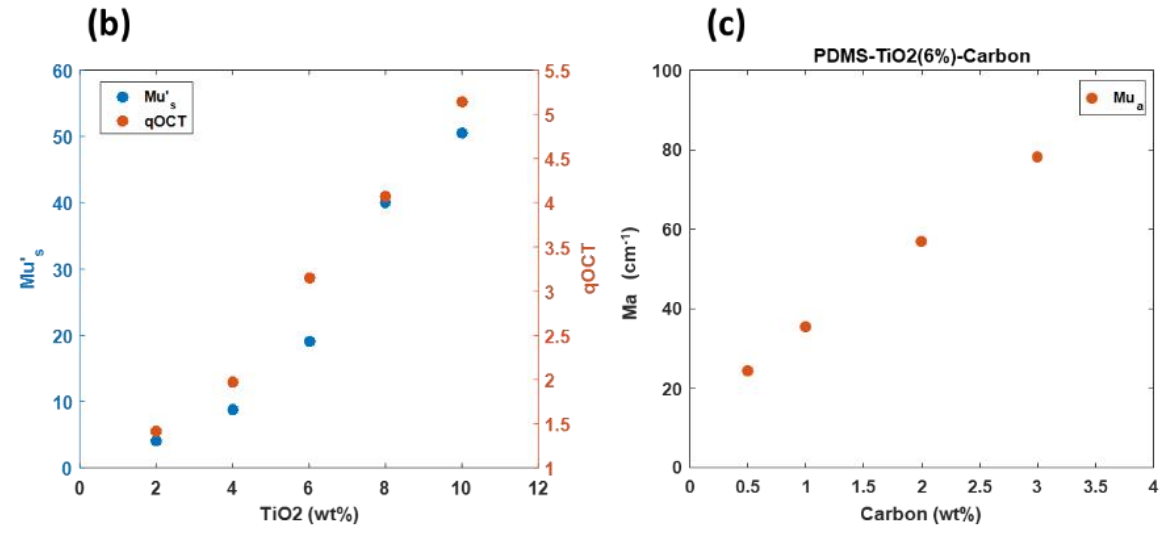

Figure 6-2 Empirical calculation of the optimum concentration of $\mathrm{TiO} 2$ and Carbon: a) 30 micron-thick PDMS-TiO2-Carbon phantoms with increasing carbon concentrations. b) Reduced scattering coefficient versus concentration of $\mathrm{TiO} 2$. c) Absorption coefficient versus Carbon concentration.

\subsubsection{Compensation of RPE melanin attenuation}

We tested the capability of the VIS-OCT-FAF system in compensation of attenuation caused by RPE melanin by making 2 layered phantoms: $1^{\text {st }}$ fluorescent scatterer layer (PDMS-TiO2(6\%wt)-RhB) with increasing Rhodamine B concentration, $2^{\text {nd }}$ absorptive layer (PDMS- Carbon) with increasing Carbon concentration to simulate the light-induced traveled melanin granules to the apical area of the RPE cells. Figure 6-3 shows the results of the quantitative imaging of these phantoms with the VIS-OCT-FAF system. 
(a)

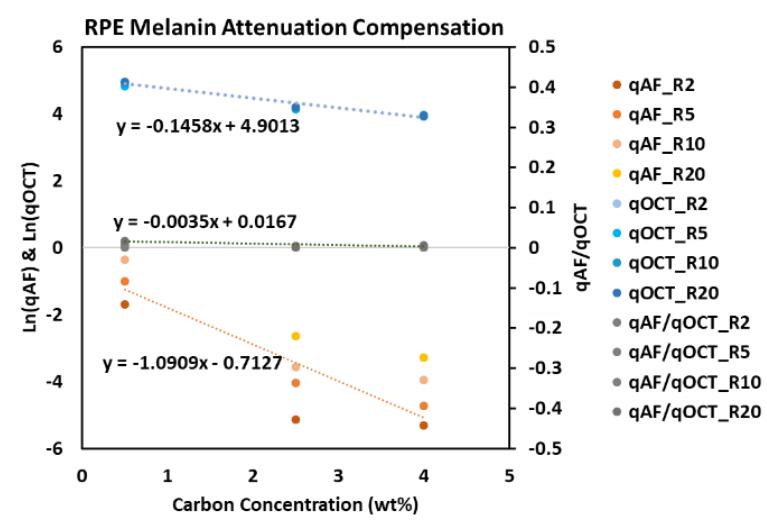

(b)

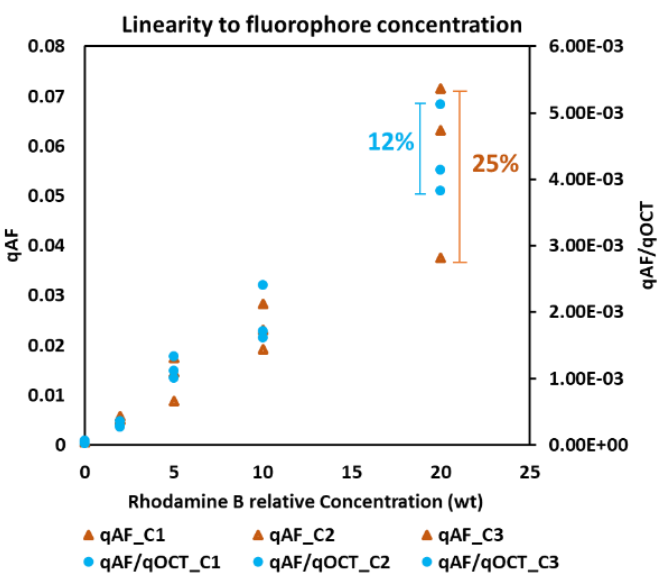

Figure 6-3 Compensation of attenuation caused by RPE melanin in phantoms: a) qAF, qOCT, and qAF/qOCT of fluorescent phantoms versus carbon concentration. b) Linear correlation of qAF and qAF/qOCT, and divergence caused by carbon attenuation.

As it is illustrated (fig. 6-3(a)), the logarithm of both qAF and qOCT linearly decreases with carbon concentration. Consequently, the ratio of $\mathrm{qAF} / \mathrm{qOCT}$ is relatively constant compared to qAF values. In other words, qAF/qOCT $(0.0035 / 0.017=21 \%)$ is less dependent on the carbon concentration compared to qAF $(1.091 / 0.713=152 \%)$. Further, for various concentrations of Rhodamine $\mathrm{B}$, both $\mathrm{qAF}$ and $\mathrm{qAF} / \mathrm{qOCT}$ values are linearly correlated with fluorophore concentration. However, different attenuation introduces more dispersion to qAF values (25\%) compared to qAF/qOCT (12\%). Based on these observations, we can conclude that qAF/qOCT is less dependent on absorbents/melanin concentration, compared to qAF alone. Therefore, qOCT can at least partially compensate for attenuation caused by RPE melanin.

\subsection{Discussion and conclusion}

We successfully fabricated fluorescent RPE simulating phantoms with similar absorption and scattering coefficients to human RPE. VIS-OCT-FAF images showed that the presence 
of absorbers decreases both VIS-OCT and FAF signals. Quantitative imaging of the fluorescent phantoms revealed that the dependency of the qAF/qOCT values to the attenuation of absorbent particles is significantly less than that of qAF values. It is worth mentioning that the OCT and AF signals of these phantoms decrease with different rates, however, the contribution of $\mathrm{qAF} / \mathrm{qOCT}$ in compensation is significant.

Although our fluorescent phantoms simulate the total scattering and absorption coefficient of the RPE layer, the distribution of scatters, absorbers, and fluorescent particles are nonhomogeneous and quite different in the RPE cells. RPE has three major scattering cushions organelles, melanosomes in the apical region, lipofuscin and melanolipofuscin in mid-cellular regions, and mitochondria basally. ${ }^{161}$ Lipofuscin and melanin granules are also fluorescent, one absorbs shorter wavelengths for excitation while the latter is excited in the NIR range. ${ }^{12}$ Apically located melanosomes attenuate FAF and OCT signals by both scattering and absorption. Substituting carbon particles with synthetic melanin might result in a more accurate simulation of the RPE layer. Further, basally located lipofuscin granules simultaneously contribute to scattering and fluorescence that is, respectively, measured by FAF and OCT. Using fluorescent microspheres that both OCT and FAF signals are originated from, instead of a combination of $\mathrm{TiO} 2$ and Rhodamine $\mathrm{B}$, can lead to a similar attenuation rate between FAF and OCT.

In conclusion, the preliminary results from RPE simulating phantoms confirm the effectiveness of the VIS-OCT-FAF method in compensating the attenuation caused by RPE melanin, emphasizing on the usefulness of this technology in clinical diagnosis. 


\section{CHAPTER 7 Quantification of Lipofusicn/A2E concentration with A2E reference target}

\subsection{Introduction}

Lipofuscin in the retinal pigment epithelium (RPE) cells, upon excitation with light in its absorption spectrum ( $488 \mathrm{~nm}$ in clinical systems), emits fluorescence known as the fundus autofluorescence $(\mathrm{FAF})^{76,77}$. FAF is thus a measure of lipofuscin concentration in the RPE, a monolayer of pigmented cells located next to photoreceptors in the retina with multiple functions ${ }^{80,162}$. Rod photoreceptors renew their entire outer segments (OS) every 10 days by adding new discs to the OS base and shedding OS tips, which are phagocytosed and digested by the RPE cells 163,164 . The daily phagocytosis of OS tips leads to the accumulation of the nondegradable end product lipofuscin, a complex lipid/protein aggregate ${ }^{67,68}$. It has been suggested that RPE lipofuscin plays a detrimental role in degenerative retinal diseases including age-related macular degeneration (AMD) and Stargardt disease ${ }^{68,165}$.

The first identified fluorophore in RPE lipofuscin is a pyridinium bisretinoid A2E (Nretinyl-N-retinylidene ethanolamine), a byproduct of the photoreceptor visual cycle and a major fluorophore of lipofuscin ${ }^{78,79,166}$. A2E has been suspected to affect RPE functions, and therapeutic strategies targeting A2E are being developed for AMD and Stargardt disease ${ }^{167,168}$.

FAF carries information of lipofuscin in the RPE cells. It is known that FAF intensities increase with age ${ }^{38}$, which reflects the accumulation of lipofuscin in the RPE with age ${ }^{87,99}$. 
FAF intensities thus can be regarded as a biomarker of RPE cell aging. Local and global alteration of FAF intensities has been seen in retinal degenerative diseases, such as AMD and Stargardt disease ${ }^{169}$. The clinical usefulness of FAF imaging is limited, however, as the currently available FAF technologies are not capable of measuring the true FAF intensities accurately. For example, it is difficult to use FAF for diagnosis and disease monitoring, since comparison of FAF images taken from different individuals or from the same individual taken at a different time requires quantitative measurement of the true FAF intensities. Technologies capable of quantitatively measuring the true FAF intensities could overcome this limitation and expand the clinical application of FAF $33,34,36-38,170-171$.

Quantitatively measuring the true FAF intensities faces two major technical challenges. The first one is to standardize the intensities of a FAF image. Delori and co-workers ${ }^{16,36,38}$ made a significant contribution in addressing this issue by placing a reference fluorescent target of known fluorescence efficiency in the intermediate retinal imaging plane. The fluorescence efficiency $\xi$ is defined as the product of the fluorophore concentration (C), the molecular quantum yield $(\mathrm{Q})$, and extinction coefficient $(\varepsilon), \quad \xi=\mathrm{C} \times \mathrm{Q} \times \varepsilon \times \mathrm{d}$. The reference target blocks a small portion of the field of view and is imaged each time a FAF image is taken. Thus, it is possible to standardize the FAF intensities using the fluorescence intensity of the reference target.

The second challenge is to compensate the signal attenuations caused by media anterior to the RPE (pre-RPE media) and melanin in the RPE. The pre-RPE media are the ocular tissues anterior to the RPE, including the cornea, the aqueous humor, the lens, the vitreous, and the neuronal retina. The pre-RPE media and RPE melanin attenuate both the excitation light before it reaches the RPE and the emitted FAF signals on their way from the RPE to 
the detector. These attenuations cannot be measured directly ${ }^{71,172}$, making compensation more difficult.

We reasoned that the depth-resolved OCT signals from the RPE could be used as a reference for signal attenuation when the OCT and FAF images are generated simultaneously from the same photons. To test this idea, we developed the VIS-OCT-FAF technology, a visible-light optical coherence tomography (VIS-OCT) based multimodal imaging technology by using a single broadband light source to generate OCT and FAF images simultaneously. We have demonstrated in our previous studies that VIS-OCT-FAF can compensate for the attenuation caused by the pre-RPE media ${ }^{31,32}$. In addition, by using a pair of fluorescent reference targets with known fluorescence efficiency and reflection coefficient inserted in the intermediate retinal imaging plane, it can also eliminate the factors like power fluctuations of the light source and gain variations of the detector ${ }^{33}$.

Here we report that signal attenuation by RPE melanin can be effectively compensated with the VIS-OCT-FAF technology using the simultaneously acquired OCT signals from the RPE as a reference. The present work is a significant step toward measuring the true FAF intensities in vivo, which could expand the clinical application of FAF to monitor retinal diseases, including disease progression and treatment outcomes.

\subsection{Method}

\subsubsection{Imaging system}

The multimodal VIS-OCT-FAF imaging system design is similar to that used in our previous publications ${ }^{32,33}$ except a single reference target was used for both fluorescence and reflection reference. The system (Fig. 6-1) consists of two single-mode optical fiber- 
based spectral-domain OCT (SD-OCT): one in the NIR used for alignment prior to imaging and the other in the visible spectrum with a center wavelength of $480 \mathrm{~nm}$ and bandwidth of $30 \mathrm{~nm}$. A superluminescent diode (SLD, center wavelength: 850nm, bandwidth: $70 \mathrm{~nm}$ ) was used for the NIR-OCT. The VIS-OCT used a supercontinuum laser (SC, SuperK Extreme EXB-6, NKT Photonics, Denmark) with a variable band-pass filter to select the center wavelength and bandwidth. The outputs of the VIS and NIR light sources were coupled into the source arms of two optical fiber-based Michelson interferometers. In the sample arms, the VIS and NIR light beams were collimated and combined by two dichroic mirrors (DM1: DMLP505, Thorlabs, and DM2: NT43-955, Edmund Optics). The combined light was scanned by an $\mathrm{X}-\mathrm{Y}$ galvanometer scanner, focused on the intermediate retinal plan by a relay lens $(\mathrm{L} 1, \mathrm{f}=75 \mathrm{~mm}$, achromatic), and delivered to the eye by an ocular lens (L2, Volk lens, 60D). At the bottom part of the field of view on the intermediate retinal-imaging plane, a PMMA reference target for FAF and OCT containing synthesized $\mathrm{A} 2 \mathrm{E}$ as the fluorescent dye is inserted.

The VIS-OCT had two reference arms split by a non-polarizing beam splitter: longer path length for retinal imaging and shorter path length for reference-target imaging. A computer-controlled shutter blocks the light in the short reference arm while the retina is scanned. When the reference target was scanned at the end of each imaging section the shutter was opened to allow the reference target to be imaged by the VIS-OCT. The combined reflected light from the sample and reference arms of NIR- and VIS-OCTs are detected by two spectrometers with the same parameters described previously ${ }^{31,32}$. 
The FAF generated by the RPE lipofuscin was detected by a PMT module (H1072320, Hamamatsu) with a $25 \mu \mathrm{m}$ diameter pinhole. After passing through the two dichroic mirrors, two long-pass filters (FGL515M, cut-on wavelength: $515 \mathrm{~nm}$, Thorlabs) and a short-pass filter (FESH0750, Cut-Off Wavelength: $750 \mathrm{~nm}$, Thorlabs), the fluorescence light was focused onto the pinhole by an $\mathrm{f}=30 \mathrm{~mm}$ achromatic doublet.

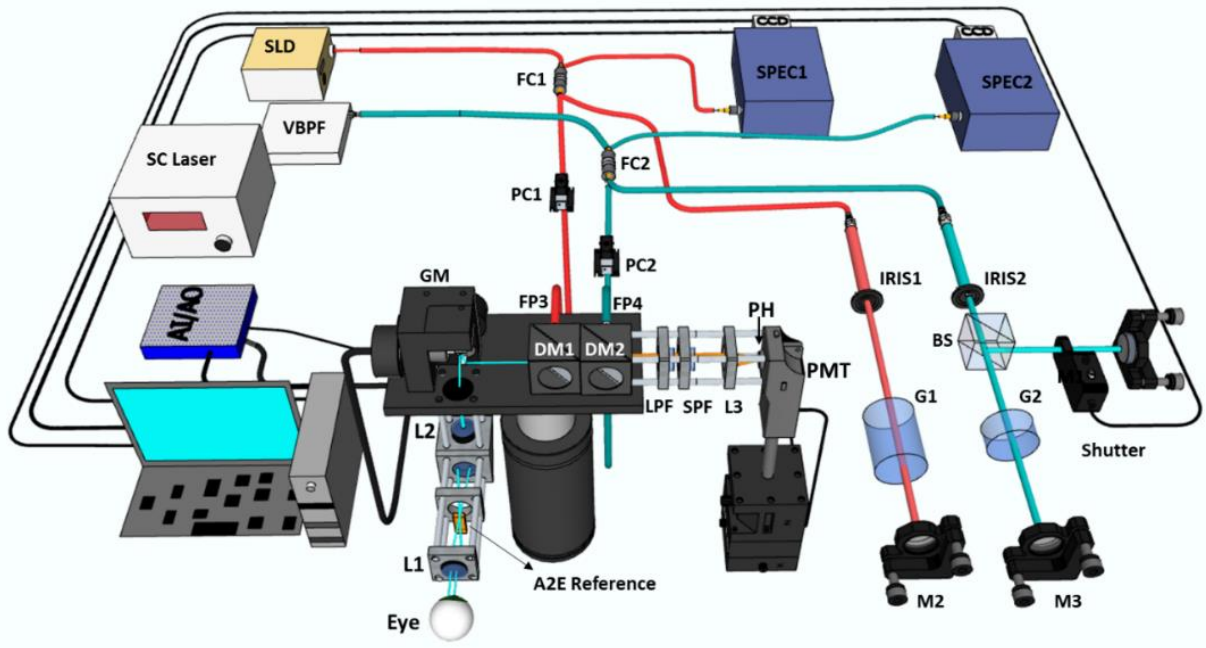

Figure 7-1 A schematic of the VIS-OCT-FAF imaging system with A2E reference. The system integrates two OCTs, a VIS-OCT (blue) and a NIR-OCT (red), and a confocal FAF detection module. In the sample arms of the two OCTs, the VIS and NIR light is combined by two dichroic mirrors (DM1 and DM2), scanned together by the X-Y galvanometer scanning mirrors (GM), and delivered to the retina by a pair of lenses (L1 and L2). The corresponding OCT signals are detected by two spectrometers (SPEC1 and SPEC2). The FAF signal is detected by a photomultiplier tube (PMT) through a set of filters and a pinhole (PH). SLD: Superluminescent Diod; SC: Supercontinuum; VBPF: Variable band-pass filter; M1-3: Reference arm mirrors; IRIS1-2: iris; G1-2: BK7 glass plates; BS: beam splitter; FC1-2: $2 \times 2$ fiber coupler; FP1-4: fiber collimator; PC1-2: polarization controller; L1-3: lens; LPF: long-pass filter; SPF: short-pass filter.

\subsubsection{Image acquisition}

The NIR-OCT real-time display was used for alignment before image acquisition. All the imaged retinas were at the same axial location on the OCT display and the optic disc was placed at the center of the raster-scan window. The NIR light was then turned off and visible light was turned on to capture VIS-OCT-FAF images. The right and left eyes of each rat were imaged four times. Each time, the alignment of the retina was slightly 
changed to test the repeatability of the results. All four images were later processed, and the average was used for quantification.

\subsubsection{In vivo imaging}

We imaged both eyes of age-matched SD and LE rats. The experiments were conducted in agreement with the ARVO (the Association for Research of Vision and Ophthalmology) Statement for the Use of Animals in Ophthalmic and Vision Research and with the guidelines of the Florida International University's Institutional Animal Care and Use Committee.

For imaging, an animal was anesthetized by intraperitoneal injection of ketamine $(54 \mathrm{mg} / \mathrm{kg}$ body weight) and xylazine ( $6 \mathrm{mg} / \mathrm{kg}$ body weight). The eye to be imaged was treated with topical proparacaine hydrochloride ophthalmic solution (Akorn, $0.5 \%$, USP) for topical anesthesia and tropicamide ophthalmic solution (Akorn, 0.5\%, USP) for pupil dilation. A hard contact lens was put on the eye to prevent corneal dehydration and opacification. The sedated rat was restrained in an animal mount with five degrees of freedom.

\subsubsection{Image processing and calculation}

The mathematical model for quantitative imaging of lipofuscin with an A2E-PMMA reference target can be expressed as:

$$
q A F=\frac{I_{F A F}}{I_{r e f-A F}}=\frac{I_{0} \tau_{p r e}^{2}(\lambda)\left[1-\rho_{\text {pre }}(\lambda)\right]^{2} \xi_{R P E} A_{d} \frac{\pi}{4} \alpha^{2}}{I_{0} \xi_{r e f-A 2 E} A_{d} \frac{\pi}{4} \alpha^{\prime 2}}
$$




$$
\begin{gathered}
=\frac{\tau_{\text {pre }}^{2}(\lambda)\left[1-\rho_{\text {pre }}(\lambda)\right]^{2} \xi_{R P E} \frac{\pi}{4} \alpha^{2}}{\xi_{\text {ref-A2E }} \frac{\pi}{4} \alpha^{\prime 2}} \\
q O C T=\frac{R_{R P E-O C T}}{R_{\text {ref-OCT }}}=\frac{I_{0} \tau_{\text {pre }}^{2}(\lambda)\left[1-\rho_{\text {pre }}(\lambda)\right]^{2} \rho_{R P E}(\lambda) \frac{\pi}{4} \alpha^{2}}{I_{0} \rho_{\text {ref-A2E }}(\lambda) \frac{\pi}{4} \alpha^{\prime 2}} \\
=\frac{\tau_{\text {pre }}^{2}(\lambda)\left[1-\rho_{\text {pre }}(\lambda)\right]^{2} \rho_{R P E}(\lambda) \frac{\pi}{4} \alpha^{2}}{\rho_{\text {ref-A2E }}(\lambda) \frac{\pi}{4} \alpha^{\prime 2}}
\end{gathered}
$$

where qAF and qOCT represent the quantified FAF and OCT signals of the RPE. IFAF and $\mathrm{I}_{\text {ref-AF }}$ are the fluorescent intensity of the fundus and the A2E reference target. $\mathrm{R}_{\mathrm{RPE}-\mathrm{OCT}}$ and $\mathrm{R}_{\text {ref-OCT }}$ are OCT reflectance signals of the RPE and Reference target, respectively. $I_{0}$ is the intensity of the incident light and $A_{d}$ represents the detection sensitivity of the PMT. $\frac{\pi}{4} \alpha^{2}$ and $\frac{\pi}{4} \alpha^{\prime 2}$ are solid angles comprising the light from the retina and reference target to the detector, respectively. $\tau_{p r e}, \rho_{\text {pre }}$, and $\rho_{\text {ref-A2E }}$ are transmittance of the pre-RPE media, the reflectance of the pre-RPE media, and reflectance of the A2E reference target, respectively. $\rho_{R P E}$ is the reflectance of the RPE layer. $\xi_{R P E}$ and $\xi_{r e f-A 2 E}$ are the fluorescence efficiency of RPE lipofuscin and $\mathrm{A} 2 \mathrm{E}$ in the reference, $\xi_{R P E}=C_{L} Q_{L} \varepsilon_{L} d_{R P E}$, $\xi_{\text {ref-A2E }}=C_{A 2 E} Q_{A 2 E} \varepsilon_{A 2 E} d_{r e f-A 2 E}$, where $\mathrm{C}, \mathrm{Q}, \varepsilon$, and $\mathrm{d}$ are concentration, quantum yield, extinction coefficient, and the effective thickness of the fluorescent sample, respectively. The subscript L represents RPE lipofuscin. We then have: 


$$
\frac{q A F}{q O C T}=\frac{C_{L} Q_{L} \varepsilon_{L} d_{R P E} / C_{A 2 E} Q_{A 2 E} \varepsilon_{A 2 E} d_{r e f-A 2 E}}{\rho_{R P E} / \rho_{r e f-A 2 E}}
$$

where $\mathrm{C}, \mathrm{Q}, \varepsilon$, and $\mathrm{d}$ are concentration, quantum yield, extinction coefficient, and the effective thickness of the fluorescent sample, respectively. The subscript L represents RPE lipofuscin. The equation can be further expressed as:

$$
\frac{q A F}{q O C T}=C F_{A 2 E} \times \frac{C_{L} Q_{L} \varepsilon_{L} d_{R P E}}{\rho_{R P E}}
$$

where $\mathrm{CF}$ is the calibration factor related to the fluorescent dye $\mathrm{A} 2 \mathrm{E}$ in the reference target.

\subsubsection{RPE-choroid preparation and lipid extraction}

Animals were euthanized immediately after imaging, and eyes were collected. The anterior segment of an eye was removed, and the retina was carefully detached and discarded. The retina was carefully dissected to obtain the RPE-choroid preparation. Total lipids were

extracted from the samples by a modified Bligh and Dyer method ${ }^{173,174}$. Briefly, the RPEchoroid preparation of each eye was mixed in $100 \mu \mathrm{l}$ of $\mathrm{H}_{2} \mathrm{O}$ with a Bullet Blender (Next Advance, Troy, NY) at setting 7 for 3 min. Methanol $(100 \mu \mathrm{l})$ was added to the sample and mixed, followed by adding $100 \mu \mathrm{l}$ of chloroform $\left(\mathrm{CHCl}_{3}\right)$ and mixing. The mixture was centrifuged at 14,000 rpm for 10 min in a tabletop microcentrifuge, and the lipid-containing lower phase was transferred to a collection tube. Extraction was repeated 4 times with 100 $\mu \mathrm{l}$ fresh chloroform added each time. Collected lipids in chloroform from each eye were 
pooled and dried in a SpeedVac (Savant Instruments, Holbrook, NY), flushed with argon, and stored at $-20^{\circ} \mathrm{C}$ in the dark until use.

\subsubsection{Quantitation of A2E by LC/MS}

The amount of A2E in each sample was measured by reverse-phase liquid chromatography-mass spectrometry (LC/MS) liquid chromatography using a Shimadzu LC system (comprising a solvent degasser, two LC-10A pumps, and an SCL-10A system controller) coupled to a Triple TOF5600 mass spectrometer (Sciex, Framingham, MA). LC was operated at a flow rate of $200 \mu \mathrm{l} / \mathrm{min}$ with a linear gradient as follows: $100 \%$ of the mobile phase A was held isocratically for 2 min and then linearly increased to $100 \%$ mobile phase B over 14 min and held at 100\% B for 4 min. Mobile phase A consisted of methanol/acetonitrile/aqueous $1 \mathrm{mM}$ ammonium acetate $(60 / 20 / 20, \mathrm{v} / \mathrm{v} / \mathrm{v})$. Mobile phase B consisted of $100 \%$ ethanol containing $1 \mathrm{mM}$ ammonium acetate. A Zorbax SB-C8 reversed-phase column (5 $\mu \mathrm{m}, 2.1$ x $50 \mathrm{~mm}$ ) was obtained from Agilent (Palo Alto, CA). The LC eluent was introduced into the ESI source of the mass spectrometer. Instrument settings for positive ion ESI/MS and MS/MS analysis of lipid species were as follows: Ion spray voltage $(\mathrm{IS})=+5500 \mathrm{~V}$; curtain gas $(\mathrm{CUR})=20 \mathrm{psi}$; ion source gas $1(\mathrm{GS} 1)=20 \mathrm{psi}$;

de-clustering potential $(\mathrm{DP})=+50 \mathrm{~V}$; focusing potential $(\mathrm{FP})=+150 \mathrm{~V}$. Data acquisition and analysis were performed using the Analyst TF1.5 software (Sciex, Framingham, MA). The measured A2E content in each sample was normalized with the amount of phosphatidylcholine 34:1 (PC 34:1), a major lipid of the retina ${ }^{175}$. 


\subsection{Results}

\subsubsection{System performance}

We built a VIS-OCT-FAF system (Fig. 6-1) for the study. The VIS-OCT-FAF system integrated a spectral-domain VIS-OCT, a spectral-domain near-infrared (NIR) OCT, and a confocal FAF detection channel on a single platform. The system used a supercontinuum light source for both VIS-OCT and FAF excitation. The filtered output of the light source had a center wavelength of $480 \mathrm{~nm}$ and a bandwidth of $30 \mathrm{~nm}$. The axial resolution and sensitivity at a path-length difference of $0.5 \mathrm{~mm}$ of the VIS-OCT were measured to be 6 $\mu \mathrm{m}$ and $85 \mathrm{~dB}$, respectively. The signal roll-off at a depth of $2 \mathrm{~mm}$ was measured to be -8 $\mathrm{dB}$, which was compensated in the quantification of the FAF intensities. The system acquires spatially registered VIS-OCT and FAF images simultaneously at a speed of $24 \mathrm{k}$ lines per second, determined by the line rate of the CCD camera of the OCT spectrometer. The NIR-OCT was used for alignment and identifying the retinal area of interest (AOI). Upon activation of data acquisition, the visible light is turned on and scanned across the AOI. The VIS-OCT and FAF images were acquired simultaneously and streamed to a computer.

\subsubsection{Characterization of the reference target}

We fabricated a reference target for both fluorescence and reflection, which was placed in the intermediate retinal imaging plane, similar to the configuration used by Delori and co-

workers $16,36,38$. The target was a piece of polymethyl methacrylate (PMMA) containing synthesized A2E as the fluorescent dye. It was made by dissolving PMMA and A2E in an anisole solvent (24 mg of A2E in $20 \mathrm{ml}$ of 4\% PMMA in anisole). The solution was cured 
by heat in an aluminum mold and the target was cut to the desired shape with a $\mathrm{CO}_{2}$ laser. The reflectance of the reference target was measured to be 0.04 , consistent with the theoretical specular reflectance of PMMA with a refractive index of 1.49. The emission spectrum of the reference target was identical to that of synthesized A2E in methanol with peak emission at $562 \mathrm{~nm}$ when measured with a custom-made spectrofluorometer at an excitation wavelength of $488 \mathrm{~nm}$. The quantum yield of A2E was measured to be $0.003 \pm 0.001$ at an excitation of $488 \mathrm{~nm}$ by using fluorescein and rhodamine solutions as references, consistent with published data ${ }^{139}$.

\subsubsection{In vivo VIS-OCT-FAF imaging}

We imaged 11-week old albino Sprague Dawley rats (SD, 3 animals, 6 eyes) and pigmented Long Evans rats (LE, 3 animals, 6 eyes) rats using the VIS-OCT-FAF system to examine the effect of attenuation compensation. Figure 6-2 shows representative quantified FAF (qAF) and VIS-OCT images. Each qAF image was obtained by normalizing the intensities of each pixel of the raw FAF image, $I_{F A F}$, to the fluorescence intensity of the reference target, $I_{\text {ref-AF }}\left(q A F=I_{F A F} / I_{\text {ref-AF}}\right.$, Eq. 3 in Methods). The fluorescence image of the reference target is shown at the bottom of each qAF image (Fig. 6-2(a) and 6-2(d)). The qAF image from an albino rat (Fig. 6-2(a)) appears brighter than the qAF image of the pigmented rat (Fig. 6-2(d)).

The en face view of the 3D OCT image (projection of the data onto the XY plane) of the albino and pigmented rats is shown in Fig. 6-2(b) and 6-2(e). The reflectance image of the reference target is shown at the bottom of Fig. 6-2(b) and 6-2(e). Cross-sectional OCT images of the albino and pigmented rats are presented in Fig. 6-2(c) and 6-2(f), respectively. 
(a) $\quad$ GAF (SD)

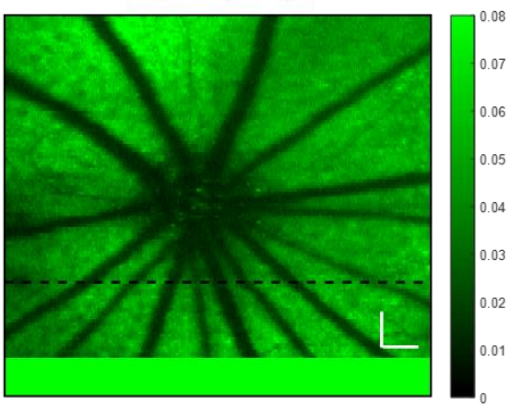

(d) QAF (LE)

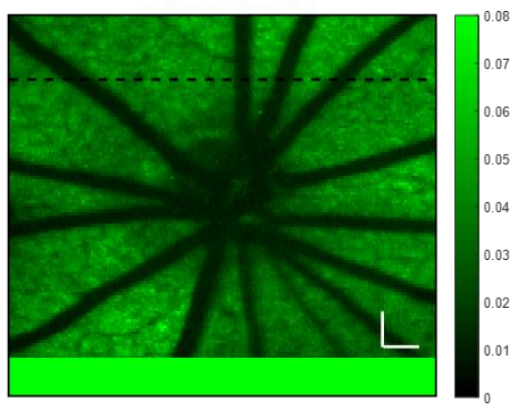

(b) OCT Fundus (SD)

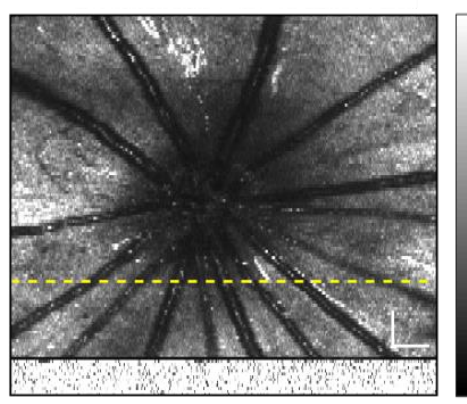

(e) OCT Fundus (LE)

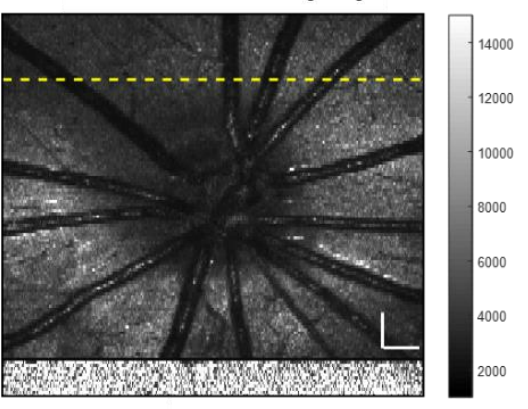

(c) OCT B-scan (SD)

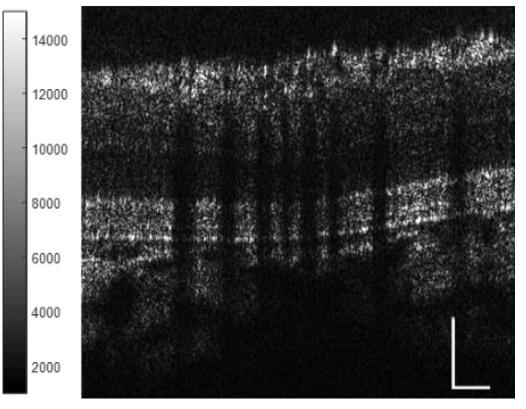

(f) OCT Fundus (LE)

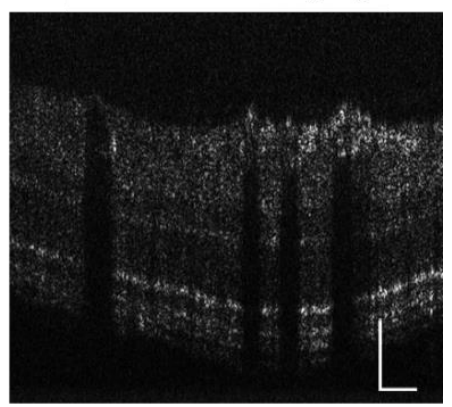

Figure 7-2 FAF and OCT images from albino (SD) and pigmented (LE) rats. The FAF and OCT images were acquired simultaneously. The intensities of the FAF $(a, d)$ were normalized to the fluorescence intensity of $\mathrm{A} 2 \mathrm{E}$ in the reference target at the bottom of each image (a and d) (see text for details). The OCT fundus images ( $b$ and e) are an en face view of the 3D OCT data projected onto the X-Y plane. The reflectance image of the reference target is shown at the bottom of each 3D OCT image (b and e). B-Scan OCT images in pannels $\mathrm{c}$ and $\mathrm{f}$ were taken at the location marked with the dashed lines, respectively. The normalized FAF intensities from the albino rat (a) appear brighter than those of the pigmented rat (d). Bars: $200 \mu \mathrm{m}$.

\subsubsection{Compensation of signal attenuation by RPE melanin}

We obtained the OCT signals of the RPE, $I_{R P E-O C T}$, by manually segmenting the RPE layer on each cross-sectional image (B-scan) and summing the signals between the two segmented boundaries (Fig. 6-3(a), red lines) that contain the RPE layer. The quantified OCT signals of the RPE (qOCT) were obtained by normalizing the raw OCT intensities to the OCT signal from the reference target $I_{r e f-O C T}: q O C T=I_{R P E-O C T} / I_{r e f-O C T}$. 
To calculate the mean of $\mathrm{qAF}$ and qOCT of each eye, a ring area centered at the optic disc was selected. It had an inner diameter of $1.38 \mathrm{~mm}$ and an outer diameter of $1.95 \mathrm{~mm}$ (Fig. 6-3(b) and 6-3(c)). The blood vessel areas are excluded from the calculation (Fig. 63(b) and 6-3(c)). The qAF, qOCT, and qAF/qOCT were calculated from this area in each of the eyes presented in Figs. 6-4 and 6-5.

(a)

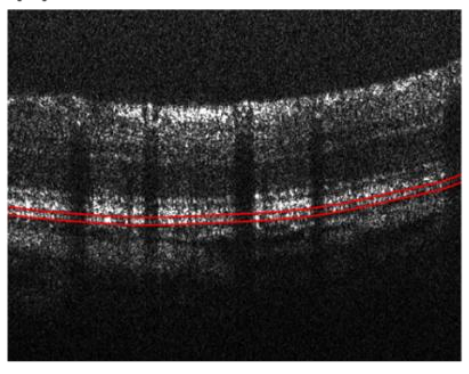

(b)

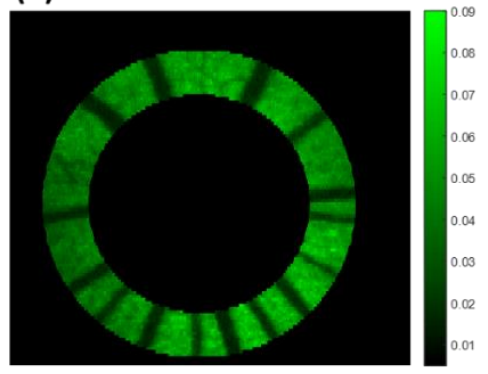

(c)

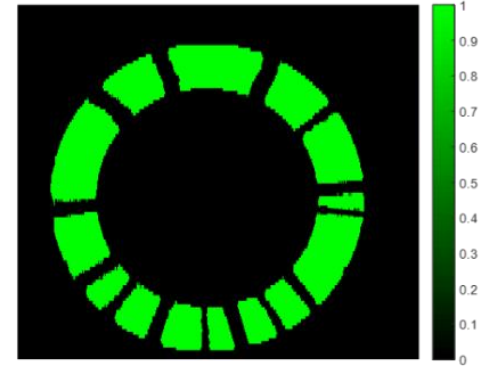

Figure 7-3 Image processing for data analysis. (a) The OCT signals from the RPE were manually segmented on the cross-sectional images between the two red lines. (b) A ring area on the fundus projection was selected to remove the optic disc and surrounding area for calculating the averaged qAF and qOCT. (c) The mask generated by binarization to remove blood vessel shadows.

The intensities of qAF and qOCT calculated from each of the 6 eyes of the 3 albino rats and 6 eyes of the 3 pigmented rats are presented in Fig. 6-4. The qAF intensities from the albino animals are higher than that from the pigmented rats (Fig. 6-4(a), yellow bars). The qOCT intensities of the albino rats are also higher than that of the pigmented rats (Fig. 4a, blue bars), apparently due to the light absorption of melanin in the RPE. When qAF was normalized to qOCT, however, the compensated FAF intensities qAF/qOCT of the pigmented animals are higher than that of the albino rats (Fig. 4a, green bars). The averaged $\mathrm{qAF} / \mathrm{qOCT}$ of pigmented rats $(67.79 \pm 16.29$, mean \pm standard deviation or $\mathrm{SD}, \mathrm{n}=6)$ is more than twice of that from the albino rats $(30.65 \pm 7.14$, mean $\pm S D, n=6, P<0.0005$, Student's t-test). The dramatic jump in readings from $\mathrm{qAF}$ to $\mathrm{qAF} / \mathrm{qOCT}$ of the pigmented 
rats demonstrates significant signal attenuation caused by RPE melanin and the compensation effect by normalizing qAF to qOCT.

(a)

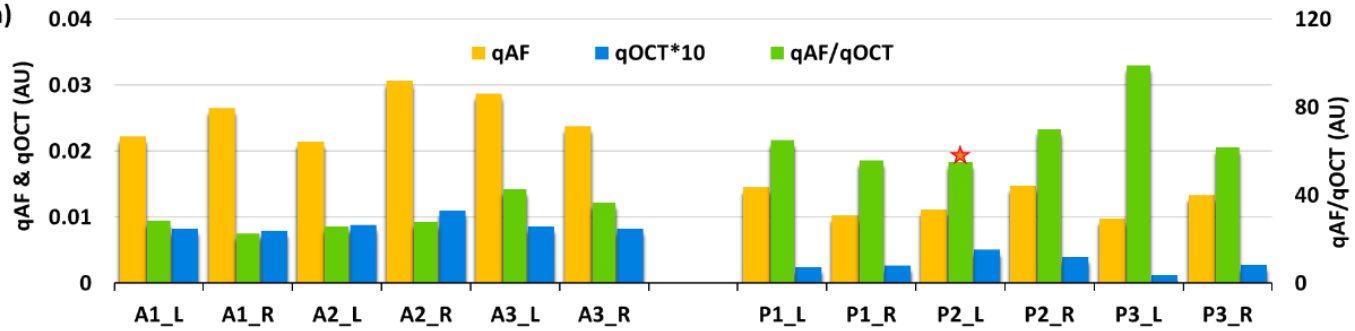

(b)

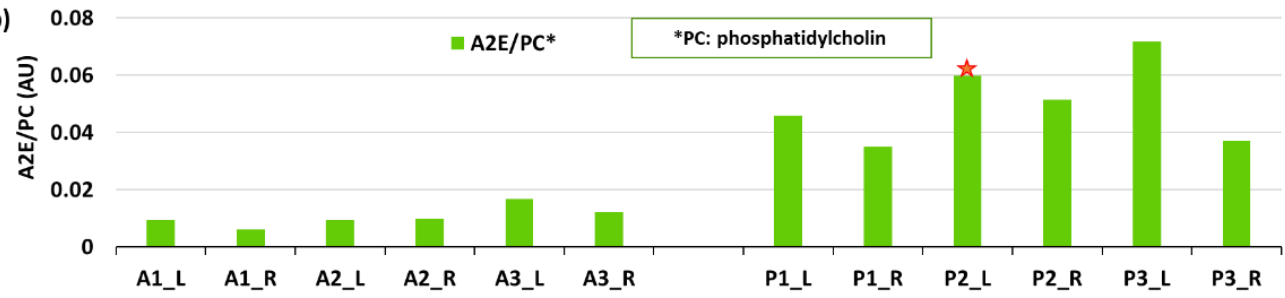

(c)

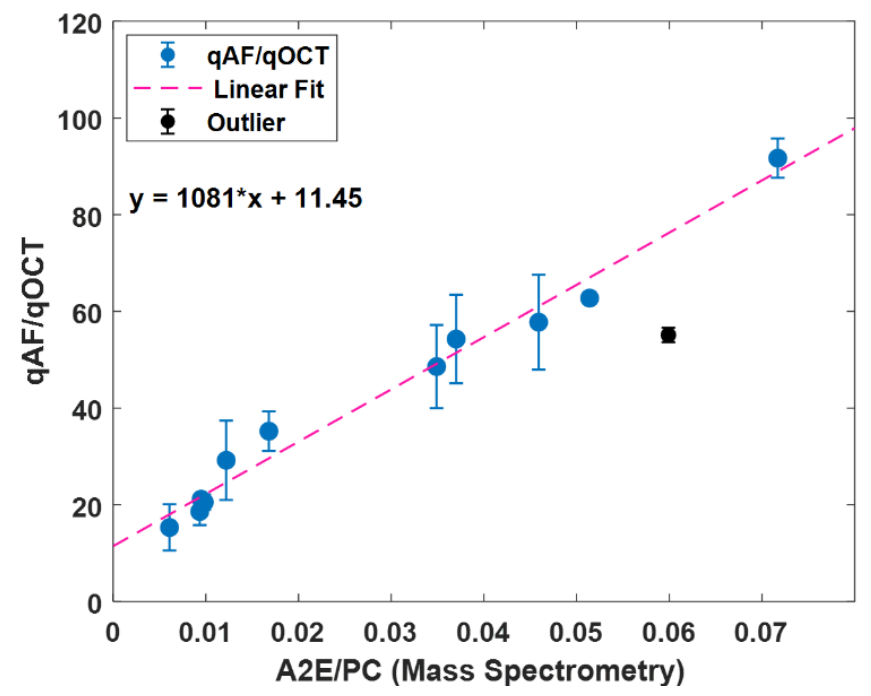

Figure 7-4 Correlation between FAF intensities and A2E amounts. Data presented are from 6 eyes of 3 albino rats (A1, A2, and A3; L: left eye; R: right eye), and 3 pigmented rats (P1, P2, and P3; L: left eye; R: right eye). The FAF intensities qAF (a, yellow bars) from the eyes of SD rats are higher than that of LE rats, so are the intensities of qOCT ( $\mathrm{a}$, blue bars). When the qAF is normalized to qOCT, the qAF/qOCT is actually higher in the pigmented LE rats than in the albino rats (a, green bars), which are in good agreement with the A2E amounts directly measured by LC/MS (b). There is a linear correlation between qAF/qOCT (mean \pm Std) and A2E (R2=0.98) (c). The qOCT values presented are multiplied by $10(\mathrm{qOCT} \times 10)$ due to their low levels. *: outlier; AU: arbitrary unit.

The higher qAF/qOCT in pigmented rats should correspond to higher concentrations of lipofuscin fluorophores in the RPE. To confirm this, we measured the A2E content of 
the same eyes by LC/MS. Eyes were collected immediately after imaging. The RPEchoroid preparation was dissected from each eye and the total lipids were extracted. The measured A2E content by LC/MS in each sample was normalized to the amount of phosphatidylcholine 34:1 (PC 34:1), a major phospholipid of the retina ${ }^{175}$. As shown in Fig. 6-4(b), A2E contents in the pigmented rats are indeed higher than that in the agematched albino rats, in good agreement with the calculated qAF/qOCT. A linear relationship was revealed between $\mathrm{qAF} / \mathrm{qOCT}$ and the A2E contents measured by LC/MS $\left(\mathrm{R}^{2}=0.98\right.$, Fig. 6-4(c)). These experimental results confirmed that qOCT is a valid reference for compensation of signal attenuation by melanin in the RPE cells, and that qAF/qOCT reliably represents the amount of lipofuscin fluorophores in the RPE, independent of signal attenuations by RPE melanin and the pre-RPE media.

To further validate the quantitative relationship between $\mathrm{qAF} / \mathrm{qOCT}$ and the $\mathrm{A} 2 \mathrm{E}$ content, we imaged three pigmented LE rats (4-month-old, 4 eyes) and three age-match albino SD rats (4 eyes) using a commercially available PMMA slide with DAPI fluorescent dye (Fluor-Ref, Microscopy Education) as the reference target. Consistent with the results shown in Fig. 6-4, the compensated FAF intensities qAF/qOCT are higher in the 4 eyes of the LE rats than in the 4 eyes of the SD rats (Fig. 6-5(a)). The A2E contents measured by LC/MS are also higher in the pigmented rats (Fig. 6-5(b)). Linear regression shows a linear relationship between qAF/qOCT and A2E content measured by LC/MS $\left(\mathrm{R}^{2}=0.95\right.$, Fig. 6$5(\mathrm{c})$ ). 

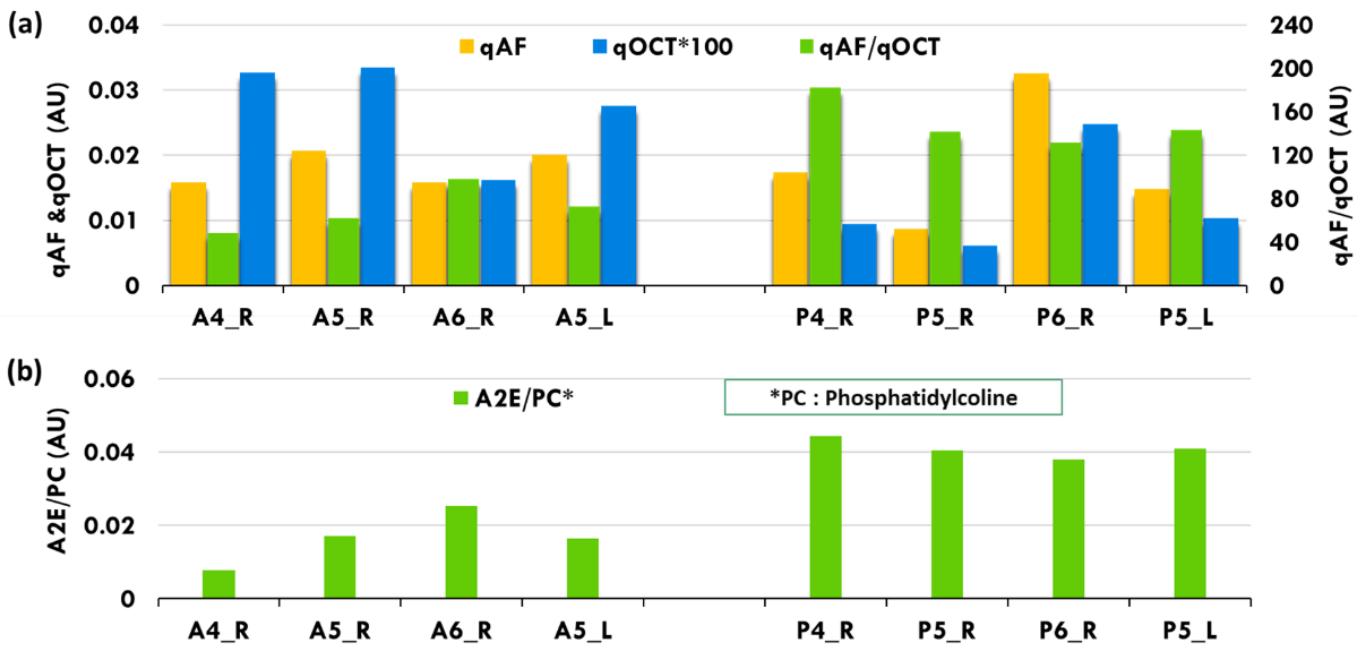

(c)

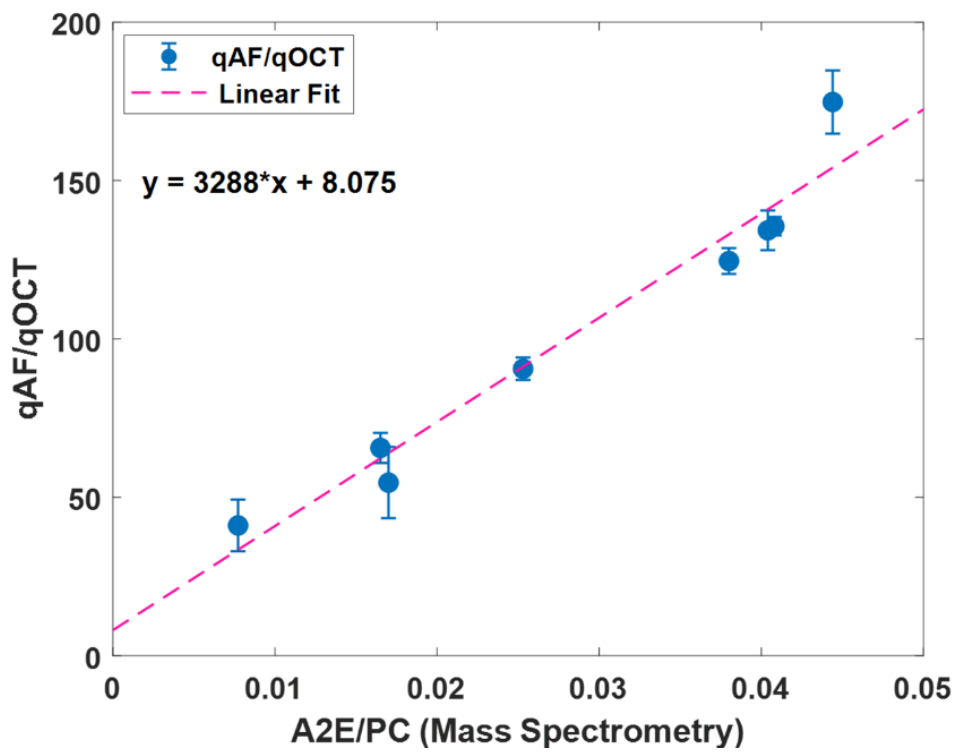

Figure 7-5 Correlation between FAF intensities and A2E amounts with a DAPI reference target. Experiments were similar to those presented in Fig. 4, except that the reference target was a commercially available PMMA slide with DAPI fluorescent dye. Similar to the results presented in Fig. 4, the qAF and qOCT of SD rats are higher than that from LE rats (a, blue and yellow bars, respectively), whereas the compensated FAF intensities $\mathrm{qAF} / \mathrm{qOCT}$ are higher in the pigmented LE rats (a, green bars). The A2E amounts, directly measured by LC/MS, are again in good agreement with qAF/qOCT (b). A linear correlation exists between $\mathrm{qAF} / \mathrm{qOCT}($ mean $\pm \mathrm{Std})$ and A2E $(\mathrm{R} 2=0.95)(\mathrm{c})$.

The slope of the fitted line for qAF/qOCT vs A2E content measured by LC/MS shown in Figs. 6-4(c) and 6-5(c) can be defined as a calibration factor $(C F)$, which is determined by the quantum yield, molar extinction coefficient, and concentration of the particular fluorescent dye in the reference target, as well as the detection efficiency of the imaging 
system. The ratio between $C F$ s of two different fluorescent dyes in the reference target, such as DAPI and A2E, can be calculated as:

$$
\frac{C F_{D A P I}}{C F_{A 2 E}}=\frac{Q_{A 2 E} C_{A 2 E} \varepsilon_{A 2 E} \eta_{A 2 E}}{Q_{D A P I} C_{D A P I} \varepsilon_{D A P I} \eta_{D A P I}}
$$

where $\mathrm{Q}, \varepsilon, \mathrm{C}$, and $\eta$ are the quantum yield, the extinction coefficient, concentration of the fluorescent dye in the reference target, and the detection efficiency of the imaging system. The subscripts A2E and DAPI specify the corresponding reference target. According to Beer's law, $A b s=\varepsilon C d$, where $A b s$ is the absorbance and $d$ is the thickness of the reference target. To calculate the ratio $C F_{D A P I} / C F_{A 2 E}$, we used a spectrophotometer to measure the absorbance of each target in the band of the illumination light $(465-495 \mathrm{~nm}){ }^{33}$. Since the detection efficiency of the imaging system for the two reference targets is the same, we have

$$
\frac{C F_{D A P I}}{C F_{A 2 E}}=\frac{Q_{A 2 E} \int_{465}^{495} A b s_{A 2 E} d \lambda}{Q_{D A P I} \int_{465}^{495} A b s_{D A P I} d \lambda}=2.88
$$

In the calculation, $\int_{465}^{495} A b s_{A 2 E} d \lambda$ and $\int_{465}^{495} A b s_{D A P I} d \lambda$ are measured to be 51.35 and 1.58, the quantum yields of 0.003 for synthesized A2E and 0.04 for DAPI ${ }^{176,177}$ were used.

The $C F_{D A P I} / C F_{A 2 E}$ ratio, calculated by using the slopes of the fitted lines shown in Fig. 6-4(c) and Fig. 6-5(c), is 3.04, close to the theoretical calculation shown in Eq. 2 with a difference of 5.5\%. These results verified our theoretical analysis. 


\subsection{Discussion}

We have developed a VIS-OCT-FAF technology to quantitatively measure the true FAF intensities in vivo by compensating the signal attenuations caused by RPE melanin and the pre-RPE media. It is based on the idea that when the FAF and OCT images are generated simultaneously by the same light source, the depth-resolved OCT signals from the RPE undergo the same signal attenuations. Thus, the OCT signals from the RPE can serve as an attenuation reference, and the attenuation caused by the RPE melanin and the pre-RPE-media can be removed by normalizing the FAF intensities to the OCT signals of the RPE. This approach does not need to measure the attenuations directly, as they are not directly measurable ${ }^{71,172}$. The compensated FAF intensities are free of attenuation caused by RPE melanin, as demonstrated by the present work, and by the pre-RPE media, as shown in our previous studies ${ }^{31,32}$. The VIS-OCT-FAF technology, in combination with a reference fluorescent target with known fluorescence efficiency and reflection coefficient placed in the intermediate retinal imaging plane, could lead to standardized accurate measurement of FAF intensities.

We used albino SD rats as a melanin negative control in the present work. SD rats, like many other albino animals, carry a missense mutation in the TYR gene that encodes a key enzyme, tyrosinase, for melanin biosynthesis ${ }^{178,179}$, resulting in the lack of melanin and albinism. It is remarkable to see that the initially measured quantitative FAF intensities qAF of the pigmented LE rats were lower than that of the age-matched SD rats, whereas, after normalization with qOCT, the compensated FAF intensities qAF/qOCT of the LE rats are actually higher than the albino rats (Fig. 6-4(a), 6-5(a)). This is rather unexpected as 
we thought the albino rats, with no protection of melanin against light exposure, could have higher amounts of A2E and thus higher FAF intensities since light is believed to be essential to $\mathrm{A} 2 \mathrm{E}$ formation ${ }^{77,136}$. The higher $\mathrm{qAF} / \mathrm{qOCT}$ of pigmented rats is confirmed by direct LC/MS measurement of A2E contents. The linear correlation between qAF/qOCT and A2E contents (Fig. 6-4, 6-5) shows that qAF/qOCT measurements correspond to the actual amounts of A2E in the RPE cells. Thus, qAF/qOCT represents the accurate FAF intensities emitted by $\mathrm{A} 2 \mathrm{E}$ and other fluorophores in the RPE lipofuscin free of the attenuating influence of melanin and the pre-RPE media.

The clinical significance of accurate FAF intensities free of signal attenuation by the RPE melanin lies on the fact that there is a large variability in the distribution of melanin in the RPE in eyes, and a large variability from individual to individual ${ }^{87}$. In addition, RPE melanin is known to decrease with age $38,87,99,180$. Furthermore, local RPE hypopigmentation and hyperpigmentation are found to occur in the early stages of AMD. 169 Since FAF intensities are significantly reduced by RPE melanin due to signal attenuation, as demonstrated by the present work, it is difficult to compare qAF from individual to individual, and qAF from the same individual at different time points without taking the signal attenuating effect of RPE melanin into consideration. For example, qAF is known to increase with age ${ }^{87,148}$, but it is not clear how much of the increase in qAF is due to the increase in RPE lipofuscin, and how much can be attributed to the age-related decrease in RPE melanin (thus less signal attenuation by RPE melanin). The true FAF intensities free of the attenuating influence of RPE melanin and the pre-RPE media by the VIS-OCT-FAF technology could have a significant impact on the clinical usefulness of FAF. 
Our theoretical analysis shows that the slope of the linear regression of qAF/qOCT vs A2E content in the RPE measured by LC/MS, i.e. the calibration factor CF, is determined by the molecular characteristics and concentration of the fluorescent dye in the reference target. This has been confirmed by two separate experiments using two reference fluorescent targets with different fluorescent dyes. Thus, when using different reference target to image FAF, the measured $\mathrm{qAF} / \mathrm{qOCT}$ can be related to each other if the characteristics of each target are known, making data obtained using different reference targets comparable. In Fig. 6-4(c) and 6-5(c), the linear regression showed a residual qAF/qOCT when A2E/PC is zero, indicating the presence of other fluorophores in the RPE lipofuscin ${ }^{79}$.

In summary, we have developed a novel VIS-OCT-FAF multimodal imaging technology to obtain true FAF intensities. It can effectively compensate for the attenuation effects of RPE melanin and pre-RPE-media by normalizing the FAF intensities to the simultaneously acquired OCT signals of the RPE. The present work is a significant step toward the standardization of quantitative FAF for clinical application.

\subsection{Acknowledgments}

We would like to thank Dr. Rui Zhou for technical assistance in image analysis. This work was supported by NIH grants R01 EY026643, R01 EY018586, P30 EY14801, and in part by an unrestricted grant from Research to Prevent Blindness, Inc. to Bascom Palmer Eye Institute. 


\section{CHAPTER 8 Translation of VIS-OCT-FAF for clinical use}

\subsection{Introduction}

Optical coherence tomography (OCT) and fundus autofluorescence (FAF) are two important modalities in ophthalmology and are widely used for diagnosis and monitoring of disease progression and treatment. The clarity of the ocular media allows direct optical access to the posterior segment of the eye and enables OCT to provide a three-dimensional image of the retinal structure and FAF to evaluate the functional biomarkers.

Human retinal imaging OCT systems commonly utilize near-infrared light sources. However, compared to NIR-OCT, visible light OCT (VIS-OCT) offers better axial resolution and having the same illumination wavelengths as sensing spectrum of the retina makes it suitable for functional assessment of photoreceptors. VIS-OCT imaging of the human eye faces several challenges: first, the relative intensity noise of SC sources is higher than laser diodes or swept-source lasers which leads to a lower system sensitivity. Second, laser safety thresholds for ocular exposure are stricter in the visible range. Third, due to the higher scattering of the ocular media in visible light, the human eye has lower transmission therefore achievable imaging depth is more limited. Forth, the human retina is sensitive to the visible light, which challenges the fixation of the eye and introduces motion artifacts. The first human retinal imaging with VIS-OCT by Yi et al ${ }^{181}$, was guided by scanning laser ophthalmoscope (SLO) utilizing a free-space interferometer, and a threedimensional translation headrest. Optimization of system sensitivity was later accomplished by controlling the reference arm power, pulse reputation, and line rate in a 
fiber-based system on an ophthalmoscope platform ${ }^{182}$. Recently, in 2019, Shu et al. ${ }^{183}$ developed a portable and optimized VIS-OCT for clinical setting with fellow-eye LED fixation target and a camera for real-time imaging of the pupil for alignment. Other studies emphasized on improvement of the image quality with minimizing chromatic and spherical aberrations by using doublet lenses. Further, software developments for axial motion tracking and spatially dependent numerical dispersion compensation have also been proved to be effective. ${ }^{184}$

In this study, taking advantage of the previous human retinal VIS-OCT systems, we modify our VIS-OCT-FAF for human retinal imaging. Significant modifications include limiting the acquisition time to less than a second by increasing the line rate to $70 \mathrm{kHz}$ to minimize motion artifacts; adjustable sample arm mount on an ophthalmoscope platform with four degrees of freedom with a vertical translational head and chinrest; Same eye fixation with NIR light scan in a shape of red cross; light safety considerations based on ANSI 2014; A shutter controlled by the software that blocks the light during alignment with NIR-OCT system and after image acquisition to minimize visible light exposure. We successfully tested the VIS-OCT-FAF system and the method of lipofuscin quantification on 10 healthy volunteers.

\subsection{Methods}

\subsubsection{VIS-OCT-FAF for human imaging}

Figure 7-1 shows the setup of the human imaging system. The VIS-OCT-FAF system is basically similar to the previous publication ${ }^{186}$. The system includes two fiber-based Michelson-interferometry SD-OCT systems: one works with NIR light using a Super 
Luminescent Diode (SLD, center wavelength: 850nm, bandwidth: $70 \mathrm{~nm}$ ); and the other one with visible spectrum centered at $480 \mathrm{~nm}$ with $30 \mathrm{~nm}$ bandwidth using a supercontinuum laser (SC, SuperK Extreme EXB-6, NKT Photonics, Denmark) with a variable band-pass filter is used for VIS-OCT light source. The NIR-OCT is used for the alignment of the retina prior to the imaging to minimize visible light exposure. Further, the center of the cross in $\mathrm{x} / \mathrm{y}$-scan of the NIR light serves as the same-eye fixation target for human subjects. A Labview software controls NIR-OCT alignment and VIS-OCT-FAF acquisition. The acquisition order by the operator enables the shutter at the visible light source. The output of the NIS and VIS lights is coupled to the source arm of two singlemode interferometers. In the sample arm, NIR and VIS lights are collimated by reflective collimators and combined by two dichroic mirrors (DM1: NT43-955, Edmund Optics, DM2: DMLP505, Thorlabs). The combined beam is scanned by an X-Y galvanometer and focused on the intermediate retinal imaging plane, where the A2E reference target is implemented, by a relay lens ( $\mathrm{L} 1, \mathrm{f}=90 \mathrm{~mm}$, achromatic). The light is then collimated and delivered to the eye by an ocular lens (L2, Volk lens, $60 \mathrm{D}$, aspheric). The bottom part of the field of view (13\% of FOV) is covered by the A2E target. The power of the VIS and NIR before the eye was measured $600 \mu \mathrm{W}$ and $800 \mu \mathrm{W}$, respectively. A vertical translational chin- and head-rest is placed in front of the eyepiece for human subject alignment. The OCT(s) sample arms and FAF detection arm are placed on an adjustable ophthalmoscope platform with 6 degrees of freedom. 


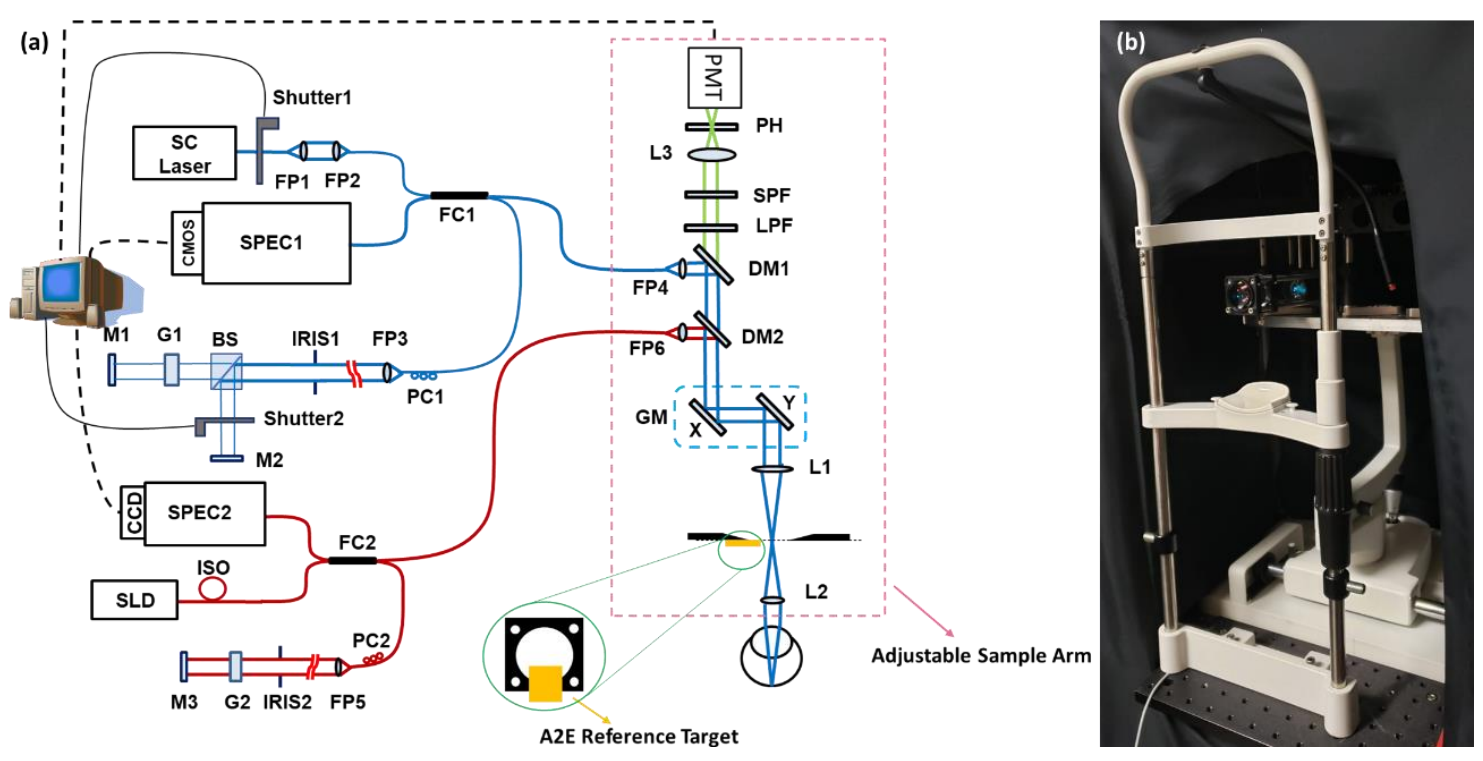

Figure 8-1 VIS-OCT-FAF for human retinal imaging: a) Schematic of VIS-OCT-FAF, b) Setup of the human retinal imaging VIS-OCT-FAF in BPEI

The reference arm of the VIS-OCT is split into two longer and shorter arms by a nonpolarizing beam splitter for imaging of the retina and $\mathrm{A} 2 \mathrm{E}$ target, respectively. A shutter controlled by the computer, block the light in the shorter arm while the retina is being scanned and is enabled when the scanning beam reaches the A2E reference location. The interference light from the sample and reference arms of NIR is detected by a spectrometer with the same parameters as previous publications ${ }^{32}$. VIS-OCT utilizes a spectrometer that has an 1800 lines/mm transmission grating, a multi-element imaging lens ( $\mathrm{f}=150 \mathrm{~mm})$, and a line scan CMOS camera (spL4096-140k, dual-line 4096 pixels with $10 \mu \mathrm{m}$ pixel size operating in 12-bit mode and vertical adding). A line-scan rate of $70 \mathrm{kHz}$ was used for the acquisition of $512 \times 128$ scanning points on the FOV, resulting in a total acquisition time of 94 milliseconds.

The FAF light is separated from the reflected excitation light by the second dichroic mirror (DM2) and passes through a long-pass filter (FGL515M, cut-on wavelength: $515 \mathrm{~nm}$, 
Thorlabs) and a short-pass filter (FESH0750, Cut-Off Wavelength: $750 \mathrm{~nm}$, Thorlabs) before being focused on the center of a $25 \mu \mathrm{m}$ diameter pinhole (Thorlabs) by an achromatic doublet $(\mathrm{f}=30 \mathrm{~mm})$. FAF is detected by a PMT module (H10723-20, Hamamatsu) after the pinhole. A multifunction data acquisition board (DAQ, PCIe-6361, National Instruments) was used to digitize the output of the PMT at a sampling rate of $3.55 \mathrm{M} / \mathrm{s}$. At each scanning position on the retina, a total of 50 points were acquired and the average of AF signals of these 50 points formed a single pixel of the AF image. FAF data acquisition, scanning of the galvanometer scanner and the OCT image acquisition was synchronized by the multifunction DAQ board.

\subsubsection{Light Safety Consideration}

American National Standards Institute (ANSI) determines a Maximum Permissible Exposure (MPE) based on the damage thresholds for a different combination of wavelengths, exposure duration, and retinal spot size that was experimentally investigated. Light exposure of the retina can introduce different kinds of damages including Thermal, Thermoacoustic, and Photochemical. Thermal damage is introduced temperature increase caused by absorption of the light by RPE melanin. For exposure duration of more than 20 $\mu$ s, the damaging energy has a less than linear dependence $\left(t^{0.75}\right)$ to the exposure time. Thermoacoustic damage happens when the pulse is shorter than $1 \mathrm{~ns}$ and photochemical damage occurs in short visible wavelengths with more than 1s exposure time that will introduce photo-oxidation to the photoreceptors and lipofuscin of RPE ${ }^{222}$. Considering the $\mathrm{MHz}$ repetition rate of the $\mathrm{SC}$ laser and normal dispersion induced by first crystal fiber and second single-mode fiber, the illumination pulse is stretched in the nanosecond scale, thus 
thermoacoustic damage can be neglected ${ }^{182}$. According to the ANSI Z136.1 - 2014 standard ${ }^{187}$, we treat the line scan exposure as an extended light source in the 400-600nm wavelengths category. With the enhanced acquisition speed, each acquisition takes less than 1 second for a square FOV, and corrections due to the use of dilation and fixation procedure do not apply to this experiment. Assuming the average focal length of the human eye $\left(f_{e}=1.7 \mathrm{~cm}\right)$ and $6 \mathrm{~mm}$ extent of the illumination area on the retina, we can calculate the full angle in radian ${ }^{185}$ as:

$$
\alpha=2 \tan ^{-1}\left(\frac{d r}{2 f e}\right) \approx \frac{d r}{f e}=0.35 \mathrm{rad}=350 \mathrm{mrad}
$$

Therefore, MPE for extended source can be calculated as:

$$
\begin{gathered}
M P E_{\text {Thermal }(1 s \leq t<100 s)}=1.8 C_{E} t^{0.75} \times 10^{-3}\left(\mathrm{~J} \cdot \mathrm{cm}^{-2}\right) \\
M P E_{\text {Photochemical }(1 s \leq t<100 s \& \alpha=350)}=7.85 \times 10^{-5} \times C_{B} \times \alpha^{2}\left(\mathrm{~J} . \mathrm{cm}^{-2}\right)
\end{gathered}
$$

According to Table $6 \mathrm{a}$ and $6 \mathrm{~b}$ in ANSI-2014, $\mathrm{C}_{\mathrm{B}}$ and $\mathrm{C}_{\mathrm{E}}$ are calculated as:

$$
\begin{gathered}
\alpha_{\max }(0.25 s \leq t)=100 \mathrm{mrad}, \alpha_{\min }=1.5 \mathrm{mrad} \\
C_{E}\left(\alpha=350 \geq \alpha_{\max }\right)=\alpha^{2} /\left(\alpha_{\min } \alpha_{\max }\right)=816.67 \\
C_{B}(\lambda=480)=10^{0.02(\lambda-450)}=3.981
\end{gathered}
$$

Therefore, we have: 


$$
\begin{gathered}
M P E_{\text {Thermal }(1 s \leq t<100 s)}=8.26\left(\mathrm{~J} \cdot \mathrm{cm}^{-2}\right) \\
M P E_{\text {Photochemical }(1 s \leq t<100 s)}=38.27\left(\mathrm{~J} \cdot \mathrm{cm}^{-2}\right)
\end{gathered}
$$

The minimum MPE is defined by Thermal MPE $\left(8.26 \mathrm{~J} . \mathrm{cm}^{-2}\right)$ for the square area of $1 \mathrm{~cm}^{2}$; for 10-time acquisition (10 seconds illumination), maximum permissible power is $0.8 \mathrm{~mW}$ which is more than the human retinal imaging power of VIS-OCT-FAF.

\subsubsection{Human Subjects}

Our study was approved by the Florida International University and the University of Miami Institutional Review Boards. We recruit 10 healthy volunteers with racial diversity (White, Asian, Hispanic, African American) between 25 and 62 years of age. We obtained informed written consent from all the subjects before their participation. We asked the subjects to sit and stabilize their head on the head and chinrest. They were asked to focus their eyes on the center of the red cross made by X and Y scanning of the NIR light so that the fovea of all volunteers was located at the center of the FOV. We aligned all the retinas at the same $\mathrm{x}, \mathrm{y}$, and $\mathrm{z}$ location by NIR-OCT. The subjects were asked to blink once and remain steady for VIS-OCT-FAF acquisition. Each eye was imaged 4 times for total exposure of less than 4 seconds and the best quality image was later used for quantification.

\subsection{Results}

We measured lipofuscin concentration in racially diverse adults of different ages. We referred to the known linear regression of lipofuscin accumulation with age to validate our in vivo quantification method using the VIS-OCT-FAF system. Images were acquired with

a FOV of $6 \mathrm{~mm}^{2}$ that was covered by a 512 (horizontal) $\times 128$ (vertical) scanning pattern. 
Figure 8-2 shows simultaneous FAF, RPE segmented OCT projection, and a crosssectional B-Scan marked with a line on the fundus projection images. RPE projection was achieved by manually segmenting the RPE boundaries on the B-Scans and summation of the signal between the boundary surfaces containing the RPE layer in the z-axis. Higherresolution imaging with 2048 A-lines was also tested on the human retina which is illustrated in figure 8-3 where RPE boundaries are clearly visible.

(a)

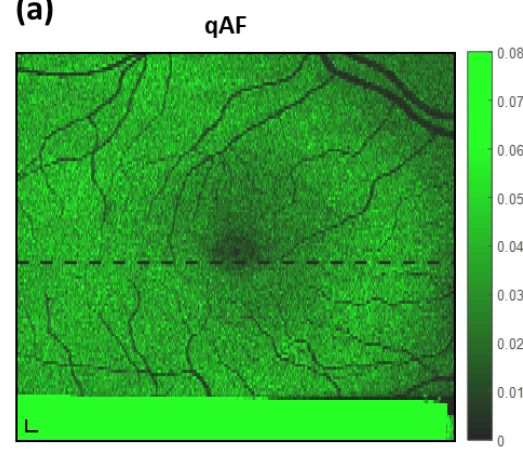

(b)

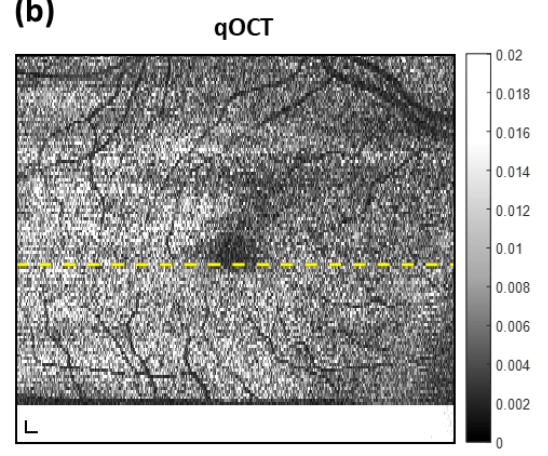

(c)

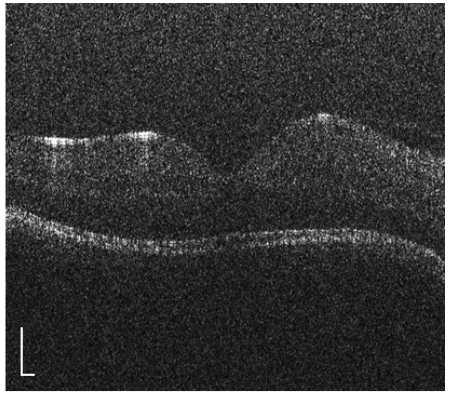

Figure 8-2 Simultaneous VIS-OCT and FAF of a 30-year-old female volunteer: a) FAF, b) en face view of segmented RPE projection, c) cross-sectional B-scan (512 A-lines) of the locations marked on (a \&b). Bar: $200 \mu \mathrm{m}$

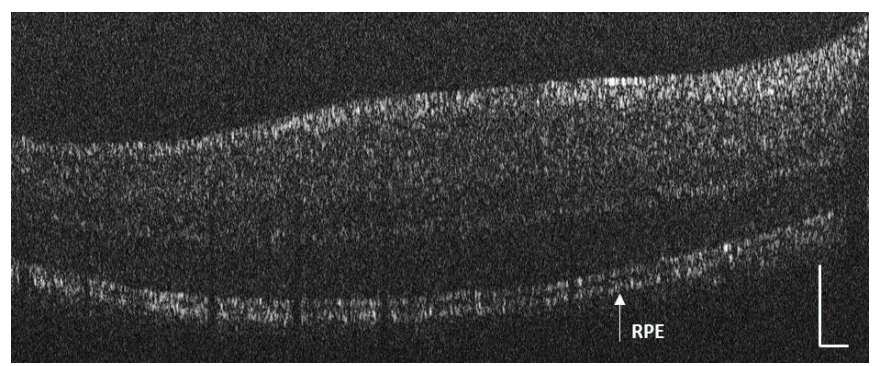

Figure 8-3 Cross-sectional B-scan with 2048 A-lines of a 55-year-old male volunteer. Bar: $200 \mu \mathrm{m}$

We used the previously introduced method for quantification of qAF, qOCT, and $\mathrm{qAF}_{\mathrm{qOCT}}{ }^{99}$. Briefly, $\mathrm{qAF}$ is achieved by normalizing the AF of the retina to that of the A2E reference, and qOCT is obtained by normalizing the OCT reflectance signal of the RPE to that of the A2E reference target. Each variable was averaged in a small window 
with the same distance from the fovea and avoiding the vessel shadows. Figure 8-4 illustrated the results of this quantification for the left and right eyes of all 10 volunteers $(n=20)$. Figure 8-5 illustrates the same data color-coded based on the ethnicity of the volunteers.

(a)

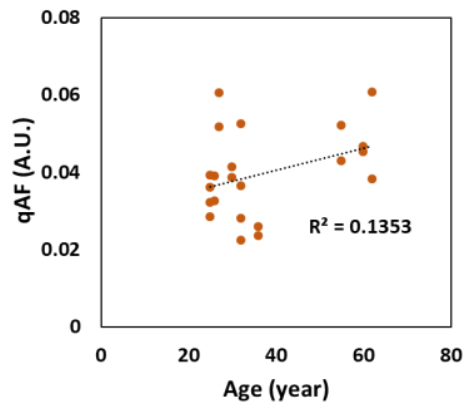

(b)

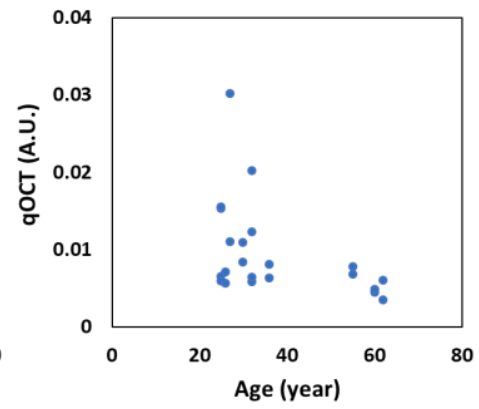

(c)

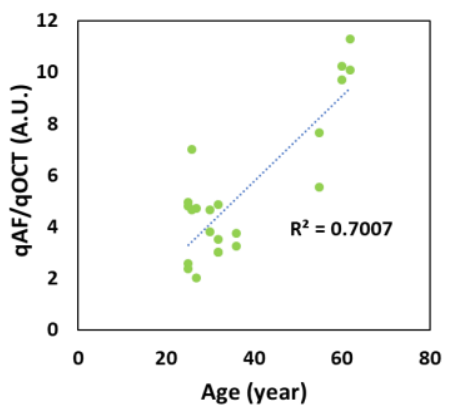

Figure 8-4 Comparison of qAF, qOCT, and qAF/qOCT of volunteers: a) qAF, b) qOCT, c) qAF/qOCT

(a)

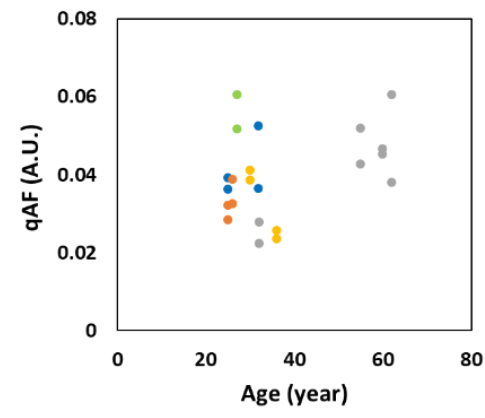

(b)

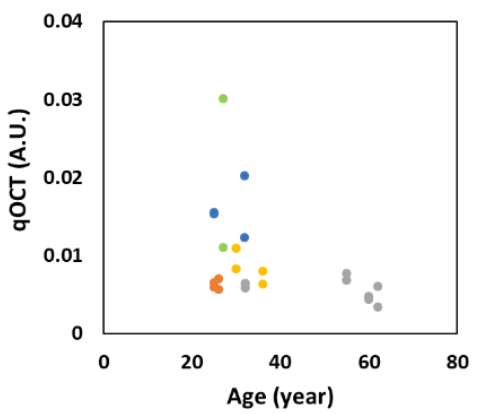

(c)

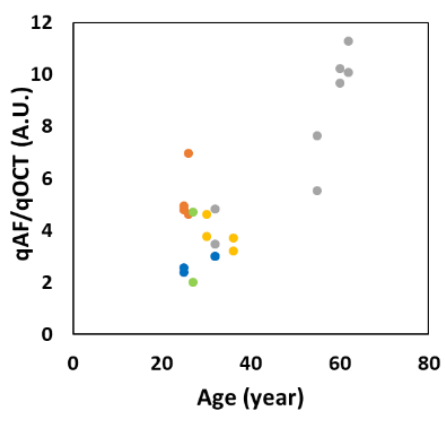

-White - Hispanic - Asian - MiddleEastern - African

Figure 8-5 Ethnicity-coded qAF, qOCT, and qAF/qOCT of volunteers: a) qAF, b) qOCT, c) qAF/qOCT A comparison of the regression coefficient between $\mathrm{qAF}$ and $\mathrm{qAF} / \mathrm{qOCT}$ values, suggests that the VIS-OCT-FAF method offers a better linear correlation with age which is consistent with previous ex vivo and in vivo studies that reported a linear increase of lipofuscin/A2E with age ${ }^{188}$. The results suggest that $\mathrm{qAF}$ values underestimate the 
lipofuscin concentration in older adults compared to $\mathrm{qAF} / \mathrm{qOCT}$ values. The reason is the fact that qOCT values in these subjects are lower than the younger group. Since our older volunteers share the same ethnicity. Whether this difference is caused by higher melanin concentration or alteration of melanin distribution with age ${ }^{188}$, is uncertain. However, according to the previous findings ${ }^{188}$, the number of apical melanin granules decreases with age, therefore, less attenuation should lead to overestimation which is not the case here. Additionally, a similar difference is observed between one Asian volunteer and other racial groups which might be because of a more significant attenuation in this ethnicity group. Further, both $\mathrm{qAF}$ and qOCT values in white volunteers were higher compared to other ethnicities with similar age groups. This was compensated in qAF/qOCT values which might be due to less significant attenuation of melanin granules in this ethnicity group. Further investigation with a bigger volunteer pool is necessary for a certain conclusion. However, these results confirm the capability of VIS-OCT-FAF system in the quantification of RPE lipofuscin/A2E

\subsection{Discussion}

We successfully prepared the VIS-OCT-FAF system for human retinal imaging in the clinic. Both OCT and FAF image quality were acceptable for RPE segmentation and quantification of $\mathrm{qAF}$ and qOCT. In vivo quantification values agree with the accumulation of RPE lipofuscin with age. Preliminary results from human retinas are consistent with quantitative results of animal models in attenuation caused by RPE melanin and capability of VIS-OCT-FAF in compensating for its effect on in vivo quantification of RPE lipofuscin. For example, both qAF and qOCT values appeared lower in the Asian group, 
and higher in White volunteers compared to the similar age other ethnicity groups. However, a higher number of volunteers is necessary for a significant conclusion. Further sample size in this study was limited and is not uniformly distributed, future investigations should consider a uniform age and ethnicity distribution of volunteers.

One of the main challenges in human retinal imaging is eye movement artifacts. Subjects often follow the visible scanning light involuntarily which might result in blocking the light beam by the pupil. In this study, we did not apply any eye drops for dilation or topical anesthesia. The application of these medications will simplify the acquisition process while limiting the MPE ${ }^{185}$. Further, lowering the illumination intensity can minimize eye sensitivity and movement artifact. This requires improvement of image acquisition sensitivity, specifically VIS-OCT through decreasing the relative intensity noise by increasing pulse repetition rate ${ }^{46,189}$.

Axial eye movement also challenges RPE segmentation and can result in an erroneous interpolation of RPE surface boundaries. This was taken into consideration during the selection of the averaging window for quantification. Axial movement correction by software-based methods like maximizing the cross-correlation, hardware-based methods such as by real-time adjustment of the reference arm ${ }^{190}$, and faster image acquisition can significantly enhance RPE segmentation. Further, the automation of segmentation software is important for future clinical studies with a higher number of subjects to speed result availability.

Another challenge in the human retinal imaging study was the focus adjustment of the ocular lens, especially for people with myopic and hyperopic vision. This was manually 
performed during the alignment procedure with NIR-OCT. However, the fine alignment of focus and automatization is necessary for clinical applications.

\subsection{Conclusion}

VIS-OCT-FAF imaging technology simultaneously acquired FAF and VIS-OCT of the human retina. Quantification of lipofuscin though qAF/qOCT values of human subjects was consistent with age accumulation of lipofuscin and confirmed the capability of the

method in attenuation compensation. The technology has significant potential in the clinical diagnosis of retinal degeneration diseases like AMD that are derived from lipofuscin accumulation in the RPE cells.

\subsection{Acknowledgment}

We would like to thank Dr. Shanhui Fan for her technical assistance in system installation in Bascom Palmer Eye Institute and image acquisition. 


\section{CHAPTER 9 Concluding Remarks}

\subsection{Summary}

Lipofuscin is a non-degradable byproduct of the visual cycle that accumulates with age and more significantly in certain pathological conditions like AMD. Excess accumulation of these fluorescent granules in the RPE cells will eventually damage RPE cells and photoreceptors, leading to retinal degeneration and irreversible blindness. Therefore, the quantification of this biomarker through quantification of true values of FAF has significant potential in clinical applications. However, interindividual and intraindividual differences in optical properties of the ocular media hurdle comparison of images from different people and from individuals at different times. Further, differences in acquisition system parameters such as the excitation illumination and sensitivity of the detector need to be standardized for the usefulness of FAF measurements.

We addressed these limitations by developing VIS-OCT-FAF with a single visible broadband light source. Since both images are generated from the same group of photons passing through the same media, VIS-OCT and FAF signals are similarly attenuated. Therefore, the reflectance signal of the RPE cells from simultaneously acquired VIS-OCT images can be used as a reference specific to persons at a specific examination time, to compensate for attenuation caused by pre-RPE media and RPE melanin. We first successfully tested the hypothesis on a model eye with a set of ND filters to simulate different attenuations in front of a fluorescent sample. In vivo imaging of rats followed in time, was consistent with the linear accumulation of lipofuscin with age. 
Further, we implemented into the VIS-OCT-FAF system, two commercially available reference targets for FAF and VIS-OCT, respectively, to compensate for fluctuations in illumination and detection parameters of the imaging system. Normalizing VIS-OCT and FAF of the model eye retina to those of the reference targets, respectively, proved the capability of the method in quantifying fluorescent efficiency independent of illumination power ad detection sensitivity, in addition to attenuation factor. Comparison in vivo study between older and albino rats revealed that qOCT was not significantly different between these two groups, while qAF and qAF/qOCT was significantly higher in older rats. Nevertheless, comparing similar age groups of albino and pigmented rats showed significantly higher qAF and qOCT values in albinos and consequently, no significant difference was observed in qAF/OCT values between albino and pigmented groups. These findings are in agreement with attenuation caused by RPE melanin that is mostly present on apical regions of RPE cells of pigmented rats and attenuates both FAF and VIS-OCT signals. However, VIS-OCT-FAF successfully compensated for the RPE melanin attenuative effect. We further confirmed this finding in the next chapters.

We later substituted two commercial references were by a single customized reference suitable for both modalities that also has similar fluorescent properties to lipofuscin for better quantification of this biomarker. The customized target consists of A2E, the major fluorophore of lipofuscin, that was synthesized with the chromatography-free method proposed by Penn et al. ${ }^{120}$, and was then dissolved in PMMA resist and cured to achieve a solid and clear target. The target was then characterized for its reflectance, absorption 
and emission spectrums, and fluorescence quantum yield. Fluorescence properties of the reference were in agreement with those of RPE lipofuscin and A2E.

To confirm the capability of the VIS-OCT-FAF in compensating for RPE melanin attenuation, we fabricated RPE simulating phantoms with similar thickness, absorption, scattering, and fluorescent characteristics. The phantoms were placed in the retinal plane of the model eye and imaged by VIS-OCT-FAF with A2E reference. As expected, both qAF and qOCT values decreased as we increased the absorbent concentration in the RPE phantoms, while qAF/qOCT values remained relatively constant.

We further validated our in vivo quantification with mass spectrometric measurement of imaged RPEs. We imaged the exact age groups of albino and pigmented rats and consistently, qAF and qOCT values appeared significantly higher in albinos, however, this time qAF/qOCT were actually higher in pigmented rats. The RPEs of these rats were then dissected and their A2E content was measured with mass spectrometry. In vivo quantification had a linear correlation with mass spec results and the slope of this correlation was dependent on the optical properties of the A2E reference target.

Following the promising results from in vivo animal studies, the system was modified for human retinal imaging. Modification mainly includes safety consideration, higher scanning rate, the mechanical design of the sample, and acquisition software for NIR-OCT alignment and VIS-OCT-FAF acquisition to minimize the visible light exposure. We imaged both eyes of 10 volunteers between 25 and 62 years of age. Our quantification results showed a better linear correlation with age in $\mathrm{qAF} / \mathrm{qOCT}$ values compared to conventional qAF. Comparison of different ethnicity groups of the same age suggests that 
RPE melanin concentration is different between these groups and was accounted for in qAF/qOCT quantification. However, larger sample size is necessary for a certain conclusion.

\subsection{Limitations and Future Work}

The first generation of VIS-OCT-FAF, although successful in lipofuscin quantification, has limitations in both hardware and software aspects. These limitations were more pronounced during human retinal imaging because of motion artifacts. The axial and lateral movement of the eye can be tracked with both hardware-based and software-based methods and then compensated either in an online or offline manner ${ }^{190}$. Due to the sensitivity of human retina to the visible light, eye motion artifacts are more significant in the visible range compare to NIR. Future work can minimize this sensitivity by implementing a fixation target, increasing the imaging speed and/or decreasing the illumination power. ${ }^{181}$

Current VIS-OCT-FAF imaging speed was limited by the camera line rate which was later enhanced from $25 \mathrm{kH}$ for in vivo animal study to $70 \mathrm{kH}$ for human study. Current setup has the potential to acquire A-lines with up to $140 \mathrm{kHz}$ speed, however, due to the trade-off between sensitivity and camera line rate, a lower speed was selected. Future work should improve imaging speed to minimize exposure and acquisition time and thus minimize eye movement artifacts and maximize patient comfort and safety.

Further, other factors such as light source stability, camera sensitivity, the chromatic and spherical aberration of the ocular media challenged the quality and resolution of the OCT images which itself hurdles the RPE segmentation process. RPE segmentation in this 
project was done semi-manually by finding the RPE boundaries of selected B-Scan and interpolating the RPE surface boundaries. The procedure can be highly time-consuming and therefore will limit the clinical usefulness of the quantification method. In future works, using doublet lenses ${ }^{183}$, and spatially dependent numerical dispersion compensation ${ }^{184}$ can significantly improve the image quality. Also, controlling the reference arm power, increasing the pulse reputation, and reducing the line rate for higher reference gray levels has previously shown to be promising for sensitivity optimization ${ }^{182}$.

In this study, we used the optic disc for consistent alignment of rats' retinas and averaged the signals in a donut-shaped region around the optic disc. However, lipofuscin distribution mapping is an important tool in clinical diagnostics ${ }^{188}$ and a real-time alignment guide software is necessary for assessment of different regions of the retina.

Due to the limited source of the synthesized A2E, RPE simulating phantoms were prepared using Rhodamine B as the fluorophore. Although the results confirmed the capability of VIS-OCT-FAF in compensating RPE melanin attenuation, it does not provide a calibration curve for human $\mathrm{A} 2 \mathrm{E}$ quantification and similar mass spectrometry measurements of human RPE is not possible except for cadaver eyes. Future work should consider providing a calibration factor for human in vivo quantification. With a large enough sample size, the technology has the potential to provide a diagnosis based on threshold quantities separating healthy and diseased retinas. 


\section{REFERENCES}

1. Jin Z, Takahashi M. Generation of retinal cells from pluripotent stem cells. In: Progress in brain research. Vol 201. Elsevier; 2012:171-181.

2. Aghaizu ND, Kruczek K, Gonzalez-Cordero A, Ali RR, Pearson RA. Pluripotent stem cells and their utility in treating photoreceptor degenerations. In: Progress in brain research. Vol 231. Elsevier; 2017:191-223.

3. Schwartz R, Loewenstein A. Early detection of age related macular degeneration: Current status. International journal of retina and vitreous. 2015;1(1):20.

4. Finnemann SC, Leung LW, Rodriguez-Boulan E. The lipofuscin component A2E selectively inhibits phagolysosomal degradation of photoreceptor phospholipid by the retinal pigment epithelium. Proc Natl Acad Sci U S A. 2002;99(6):3842-3847.

5. Kennedy CJ, Rakoczy PE, Constable IJ. Lipofuscin of the retinal pigment epithelium: A review. Eye. 1995;9(6):763.

6. Nandakumar N, Buzney S, Weiter JJ. Lipofuscin and the principles of fundus autofluorescence: A review. . 2012;27(5-6):197-201.

7. Sepah YJ, Akhtar A, Sadiq MA, et al. Fundus autofluorescence imaging: Fundamentals and clinical relevance. Saudi Journal of Ophthalmology. 2014;28(2):111116. 
8. Parmar VM, Parmar T, Arai E, Perusek L, Maeda A. A2E-associated cell death and inflammation in retinal pigmented epithelial cells from human induced pluripotent stem cells. Stem cell research. 2018;27:95-104.

9. Katz ML, Drea CM, Eldred GE, Hess HH, Robison Jr WG. Influence of early photoreceptor degeneration on lipofuscin in the retinal pigment epithelium. Exp Eye Res. 1986;43(4):561-573.

10. Robison WG, Kuwabara T, Bieri JG. Deficiencies of vitamins E and A in the rat. retinal damage and lipofuscin accumulation. Invest Ophthalmol Vis Sci. 1980;19(9):10301037.

11. Katz ML, Norberg M, Stientjes HJ. Reduced phagosomal content of the retinal pigment epithelium in response to retinoid deprivation. Invest Ophthalmol Vis Sci. 1992;33(9):2612-2618.

12. Holz F, Bird AC, Schmitz-Valckenberg S, Spaide R. Atlas of fundus autofluorscence imaging. Vol 1. Springer; 2007.

13. Bellmann C, Rubin GS, Kabanarou SA, Bird AC, Fitzke FW. Fundus autofluorescence imaging compared with different confocal scanning laser ophthalmoscopes. Br J Ophthalmol. 2003;87(11):1381-1386.

14. Artal P. Optics of the eye and its impact in vision: A tutorial. Advances in Optics and Photonics. 2014;6(3):340-367. 
15. Boettner EA, Wolter JR. Transmission of the ocular media. Invest Ophthalmol Vis Sci. 1962;1(6):776-783.

16. Delori F, Greenberg JP, Woods RL, et al. Quantitative measurements of autofluorescence with the scanning laser ophthalmoscope. Invest Ophthalmol Vis Sci. 2011;52(13):9379-9390.

17. Festner Z. Understanding multiple sclerosis through retinal cell layer thickness: An insight into the neurodegeneration process. . 2016.

18. Bruno Dubuc. The brain from top to bottom.

https://thebrain.mcgill.ca/flash/d/d_02/d_02_cl/d_02_cl_vis/d_02_cl_vis.html. Accessed 10/23, 2019.

19. Purves D, Cabeza R, Huettel SA, et al. Cognitive neuroscience. Sunderland: Sinauer Associates, Inc; 2008.

20. Tanaka M, Tachibana M. Independent control of reciprocal and lateral inhibition at the axon terminal of retinal bipolar cells. J Physiol (Lond ). 2013;591(16):3833-3851.

\section{Fitzakerley J. Physiology of the retina pigment epithelium.}

https://www.d.umn.edu/ jfitzake/Lectures/DMED/Vision/Retina/VisualCycle.html. Updated 2015. Accessed December, 2019. 
22. Crouch RK, Koutalos Y, Kono M, Schey K, Ablonczy Z. A2E and lipofuscin. In: Progress in molecular biology and translational science. Vol 134. Elsevier; 2015:449463.

23. Mazzitello KI, Arizmendi CM, Family F, Grossniklaus HE. Formation and growth of lipofuscin in the retinal pigment epithelium cells. Physical Review E. 2009;80(5):051908.

24. Winkler BS, Boulton ME, Gottsch JD, Sternberg P. Oxidative damage and agerelated macular degeneration. Mol Vis. 1999;5:32.

25. Wassell J, Davies S, Bardsley W, Boulton M. The photoreactivity of the retinal age pigment lipofuscin. J Biol Chem. 1999;274(34):23828-23832.

26. Beatty S, Boulton M, Henson D, Koh HH, Murray IJ. Macular pigment and age related macular degeneration. Br J Ophthalmol. 1999;83(7):867-877.

27. von Ruckmann A, Schmidt KG, Fitzke FW, Bird AC, Jacobi KW. Dynamics of accumulation and degradation of lipofuscin in retinal pigment epithelium in senile macular degeneration. Klin Monbl Augenheilkd. 1998;213(1):32-37.

28. Booysen DJ. A review of fundus autofluorescence imaging. African Vision and Eye Health. 2013;72(1):46-53.

29. Schmitz-Valckenberg S, Fitzke FW, Holz FG. Fundus autofluorescence imaging with the confocal scanning laser ophthalmoscope. Atlas of fundus autofluorescence imaging. 2007:31-36. 
30. Keane PA, Sadda SR. Retinal imaging in the twenty-first century: State of the art and future directions. Ophthalmology. 2014;121(12):2489-2500.

31. Nafar Z, Jiang M, Wen R, Jiao S. Visible-light optical coherence tomography-based multimodal retinal imaging for improvement of fluorescent intensity quantification. Biomedical optics express. 2016;7(9):3220-3229.

32. Nafar Z, Wen R, Jiao S. Visible light OCT-based quantitative imaging of lipofuscin in the retinal pigment epithelium with standard reference targets. Biomedical Optics Express. 2018;9(8):3768-3782.

33. Duncker T, Greenberg JP, Ramachandran R, et al. Quantitative fundus autofluorescence and optical coherence tomography in best vitelliform macular dystrophy. Invest Ophthalmol Vis Sci. 2014;55(3):1471-1482.

34. Duncker T, Tsang SH, Lee W, et al. Quantitative fundus autofluorescence distinguishes ABCA4-associated and Non-ABCA4-associated bull's-eye maculopathy. Ophthalmology. 2015;122(2):345-355.

35. Gliem M, Müller PL, Finger RP, McGuinness MB, Holz FG, Issa PC. Quantitative fundus autofluorescence in early and intermediate age-related macular degeneration. JAMA ophthalmology. 2016;134(7):817-824.

36. Burke TR, Duncker T, Woods RL, et al. Quantitative fundus autofluorescence in recessive stargardt disease. Invest Ophthalmol Vis Sci. 2014;55(5):2841-2852. 
37. Sparrow JR, Blonska A, Flynn E, et al. Quantitative fundus autofluorescence in mice: Correlation with HPLC quantitation of RPE lipofuscin and measurement of retina outer nuclear layer thickness. Invest Ophthalmol Vis Sci. 2013;54(4):2812-2820.

38. Greenberg JP, Duncker T, Woods RL, Smith RT, Sparrow JR, Delori FC.

Quantitative fundus autofluorescence in healthy eyes. Invest Ophthalmol Vis Sci. 2013;54(8):5684-5693.

39. Hammond Jr BR, Fuld K, Snodderly MD. Iris color and macular pigment optical density. Exp Eye Res. 1996;62(3):293-298.

40. Van den Berg T, IJspeert JK, De Waard P. Dependence of intraocular straylight on pigmentation and light transmission through the ocular wall. Vision Res. 1991;31(78):1361-1367.

41. Yaqoob Z, Wu J, Yang C. Spectral domain optical coherence tomography: A better OCT imaging strategy. BioTechniques. 2005;39(6):S6-S13.

42. Hee MR, Izatt JA, Swanson EA, et al. Optical coherence tomography of the human retina. Arch Ophthalmol. 1995;113(3):325-332.

43. Drexler W, Morgner U, Ghanta RK, Kärtner FX, Schuman JS, Fujimoto JG. Ultrahigh-resolution ophthalmic optical coherence tomography. Nat Med. 2001;7(4):502.

44. Povazay B, Apolonski AA, Unterhuber A, et al. Visible light optical coherence tomography. . 2002;4619:90-95. 
45. Jacques SL. Optical properties of biological tissues: A review. Physics in Medicine \& Biology. 2013;58(11):R37.

46. Shu X, Beckmann LJ, Zhang HF. Visible-light optical coherence tomography: A review. J Biomed Opt. 2017;22(12):121707.

47. Chen S, Yi J, Zhang HF. Measuring oxygen saturation in retinal and choroidal circulations in rats using visible light optical coherence tomography angiography. Biomedical optics express. 2015;6(8):2840-2853.

48. Chen S, Shu X, Nesper PL, Liu W, Fawzi AA, Zhang HF. Retinal oximetry in humans using visible-light optical coherence tomography. Biomedical optics express. 2017;8(3):1415-1429.

49. Yi J, Liu W, Chen S, et al. Visible light optical coherence tomography measures retinal oxygen metabolic response to systemic oxygenation. Light: Science \& Applications. 2015;4(9):e334.

50. Chen S, Yi J, Zhang HF. Dynamically measuring retinal oxygen saturation at microvascular level using visible-light OCT angiograph. Invest Ophthalmol Vis Sci. 2015;56(7):3308.

51. Zhang X, Hu J, Knighton RW, Huang X, Puliafito CA, Jiao S. Dual-band spectraldomain optical coherence tomography for in vivo imaging the spectral contrasts of the retinal nerve fiber layer. Optics express. 2011;19(20):19653-19659. 
52. Lichtenegger A, Harper DJ, Augustin M, et al. Spectroscopic imaging with spectral domain visible light optical coherence microscopy in alzheimer's disease brain samples. Biomedical optics express. 2017;8(9):4007-4025.

53. Muqit MM, Gray JC, Marcellino GR, et al. Fundus autofluorescence and fourierdomain optical coherence tomography imaging of 10 and 20 millisecond pascal retinal photocoagulation treatment. Br J Ophthalmol. 2009;93(4):518-525.

54. Greenberg JP, Sherman J, Zweifel SA, et al. Spectral-domain optical coherence tomography staging and autofluorescence imaging in achromatopsia. JAMA ophthalmology. 2014;132(4):437-445.

55. Xue K, Oldani M, Jolly JK, et al. Correlation of optical coherence tomography and autofluorescence in the outer retina and choroid of patients with choroideremia. Invest Ophthalmol Vis Sci. 2016;57(8):3674-3684.

56. No title. https://www.businesswire.com/news/home/20061103005206/en/HeidelbergEngineering-Receives-FDA-Clearance-Worlds-Spectral. Updated 2006.

57. Wolf-Schnurrbusch UE, Enzmann V, Brinkmann CK, Wolf S. Morphologic changes in patients with geographic atrophy assessed with a novel spectral OCT-SLO combination. Invest Ophthalmol Vis Sci. 2008;49(7):3095-3099.

58. Helb H, Issa PC, Fleckenstein M, et al. Clinical evaluation of simultaneous confocal scanning laser ophthalmoscopy imaging combined with high-resolution, spectral-domain optical coherence tomography. Acta Ophthalmol. 2010;88(8):842-849. 
59. Fleckenstein M, Schmitz-Valckenberg S, Martens C, et al. Fundus autofluorescence and spectral-domain optical coherence tomography characteristics in a rapidly progressing form of geographic atrophy. Invest Ophthalmol Vis Sci. 2011;52(6):37613766.

60. Dai C, Liu X, Jiao S. Simultaneous optical coherence tomography and autofluorescence microscopy with a single light source. J Biomed Opt. 2012;17(8):805021.

61. Huang D, Swanson EA, Lin CP, et al. Optical coherence tomography. Science. 1991;254(5035):1178-1181.

62. Wojtkowski M, Srinivasan VJ, Ko TH, Fujimoto JG, Kowalczyk A, Duker JS. Ultrahigh-resolution, high-speed, fourier domain optical coherence tomography and methods for dispersion compensation. Optics express. 2004;12(11):2404-2422.

63. Singh AD, Belfort RN, Sayanagi K, Kaiser PK. Fourier domain optical coherence tomographic and auto-fluorescence findings in indeterminate choroidal melanocytic lesions. Br J Ophthalmol. 2010;94(4):474-478.

64. Delori FC, Dorey CK, Staurenghi G, Arend O, Goger DG, Weiter JJ. In vivo fluorescence of the ocular fundus exhibits retinal pigment epithelium lipofuscin characteristics. Invest Ophthalmol Vis Sci. 1995;36(3):718-729. 
65. de Venecia G, Davis M, Engerman R. Clinicopathologic correlations in diabetic retinopathy: I. histology and fluorescein angiography of microaneurysms. Arch Ophthalmol. 1976;94(10):1766-1773.

66. Holz FG, Bellmann C, Rohrschneider K, Burk RO, Voölcker HE. Simultaneous confocal scanning laser fluorescein and indocyanine green angiography. Am J Ophthalmol. 1998;125(2):227-236.

67. Boulton M, McKechnie NM, Breda J, Bayly M, Marshall J. The formation of autofluorescent granules in cultured human RPE. Invest Ophthalmol Vis Sci. 1989;30(1):82-89.

68. Sparrow JR, Boulton M. RPE lipofuscin and its role in retinal pathobiology. Exp Eye Res. 2005;80(5):595-606.

69. Johnson EJ. Age-related macular degeneration and antioxidant vitamins: Recent findings. Curr Opin Clin Nutr Metab Care. 2010;13(1):28-33.

70. Jiang M, Liu T, Liu X, Jiao S. Simultaneous optical coherence tomography and lipofuscin autofluorescence imaging of the retina with a single broadband light source at 480nm. Biomedical optics express. 2014;5(12):4242-4248.

71. Pokorny J, Smith VC, Lutze M. Aging of the human lens. Appl Opt. 1987;26(8):1437-1440. 
72. Dai C, Liu X, Zhang HF, Puliafito CA, Jiao S. Absolute retinal blood flow measurement with a dual-beam doppler optical coherence tomography. Invest Ophthalmol Vis Sci. 2013;54(13):7998-8003.

73. van de Kraats J, Berendschot TT, van Norren D. The pathways of light measured in fundus reflectometry. Vision Res. 1996;36(15):2229-2247.

74. Morgan JI, Pugh EN. Scanning laser ophthalmoscope measurement of local fundus reflectance and autofluorescence changes arising from rhodopsin bleaching and regeneration. Invest Ophthalmol Vis Sci. 2013;54(3):2048-2059.

75. Jiao S, Knighton R, Huang X, Gregori G, Puliafito CA. Simultaneous acquisition of sectional and fundus ophthalmic images with spectral-domain optical coherence tomography. Optics express. 2005;13(2):444-452.

76. Delori FC, Staurenghi G, Arend O, Dorey CK, Goger DG, Weiter JJ. In vivo measurement of lipofuscin in stargardt's disease--fundus flavimaculatus. Invest Ophthalmol Vis Sci. 1995;36(11):2327-2331.

77. Delori FC, Bursell SE, Yoshida A, McMeel JW. Vitreous fluorophotometry in diabetics: Study of artifactual contributions. Graefe's Archive for Clinical and Experimental Ophthalmology. 1985;222(4-5):215-218.

78. Sparrow JR, Fishkin N, Zhou J, et al. A2E, a byproduct of the visual cycle. Vision Res. 2003;43(28):2983-2990. 
79. Sparrow JR, Gregory-Roberts E, Yamamoto K, et al. The bisretinoids of retinal pigment epithelium. Prog Retin Eye Res. 2012;31(2):121-135.

80. Strauss O. The retinal pigment epithelium in visual function. Physiol Rev. $2005 ; 85(3): 845-881$.

81. Kiser PD, Golczak M, Palczewski K. Chemistry of the retinoid (visual) cycle. Chem Rev. 2013;114(1):194-232.

82. Kevany BM, Palczewski K. Phagocytosis of retinal rod and cone photoreceptors. Physiology. 2010;25(1):8-15.

83. Zhang X, Jiang M, Fawzi AA, et al. Simultaneous dual molecular contrasts provided by the absorbed photons in photoacoustic microscopy. Opt Lett. 2010;35(23):4018-4020.

84. Zhang X, Zhang HF, Puliafito CA, Jiao S. Simultaneous in vivo imaging of melanin and lipofuscin in the retina with photoacoustic ophthalmoscopy and autofluorescence imaging. J Biomed Opt. 2011;16(8):080504-3.

85. Liu X, Liu T, Wen R, et al. Optical coherence photoacoustic microscopy for in vivo multimodal retinal imaging. Opt Lett. 2015;40(7):1370-1373.

86. Zhang X, Jiao S, Zhang H. Optical coherence photoacoustic microscopy: Accomplishing optical coherence tomography and photoacoustic microscopy with a single light source. J Biomed Opt. 2012;17(3):030502. 
87. Weiter JJ, Delori FC, Wing GL, Fitch KA. Retinal pigment epithelial lipofuscin and melanin and choroidal melanin in human eyes. Invest Ophthalmol Vis Sci. $1986 ; 27(2): 145-152$.

88. Sadda SR. Fundus autofluoresence imaging: Principles and applications. Retina Phys. 2011.

89. Boyer NP, Higbee D, Currin MB, et al. Lipofuscin and N-retinylidene-Nretinylethanolamine (A2E) accumulate in retinal pigment epithelium in absence of light exposure: Their origin is 11-cis-retinal. J Biol Chem. 2012;287(26):22276-22286.

90. Afridi R, Agarwal A, Sadiq MA, et al. Fundus autofluorescence imaging in posterior uveitis. In: Multimodal imaging in uveitis. Springer; 2018:69-85.

91. Wing GL, Blanchard GC, Weiter JJ. The topography and age relationship of lipofuscin concentration in the retinal pigment epithelium. Invest Ophthalmol Vis Sci. $1978 ; 17(7): 601-607$.

92. An L, Li P, Shen TT, Wang R. High speed spectral domain optical coherence tomography for retinal imaging at 500,000 a-lines per second. Biomedical optics express. 2011;2(10):2770-2783.

93. Holz FG, Bellman C, Staudt S, Schütt F, Völcker HE. Fundus autofluorescence and development of geographic atrophy in age-related macular degeneration. Invest Ophthalmol Vis Sci. 2001;42(5):1051-1056. 
94. Bindewald A, Bird AC, Dandekar SS, et al. Classification of fundus autofluorescence patterns in early age-related macular disease. Invest Ophthalmol Vis Sci. 2005;46(9):3309-3314.

95. Schmitz-Valckenberg S, Bindewald-Wittich A, Dolar-Szczasny J, et al. Correlation between the area of increased autofluorescence surrounding geographic atrophy and disease progression in patients with AMD. Invest Ophthalmol Vis Sci. 2006;47(6):26482654.

96. Yonekawa Y, Miller JW, Kim IK. Age-related macular degeneration: Advances in management and diagnosis. Journal of clinical medicine. 2015;4(2):343-359.

97. Yung M, Klufas MA, Sarraf D. Clinical applications of fundus autofluorescence in retinal disease. International journal of retina and vitreous. 2016;2(1):12.

98. Sparrow JR, Duncker T. Fundus autofluorescence and RPE lipofuscin in age-related macular degeneration. Journal of clinical medicine. 2014;3(4):1302-1321.

99. Boulton M, Dayhaw-Barker P. The role of the retinal pigment epithelium: Topographical variation and ageing changes. Eye. 2001;15(3):384-389.

100. Rudolf M, Vogt SD, Curcio CA, et al. Histologic basis of variations in retinal pigment epithelium autofluorescence in eyes with geographic atrophy. Ophthalmology. $2013 ; 120(4): 821-828$. 
101. Allingham MJ, Nie Q, Lad EM, et al. Semiautomatic segmentation of rim area focal hyperautofluorescence predicts progression of geographic atrophy due to dry age-related macular degeneration. Invest Ophthalmol Vis Sci. 2016;57(4):2283-2289.

102. Lois N, Halfyard AS, Bunce C, Bird AC, Fitzke FW. Reproducibility of fundus autofluorescence measurements obtained using a confocal scanning laser ophthalmoscope. Br J Ophthalmol. 1999;83(3):276-279.

103. Schwartz A, Wang L, Early E, et al. Quantitating fluorescence intensity from fluorophore: The definition of MESF assignment. Journal of research of the National Institute of Standards and Technology. 2002;107(1):83.

104. Weersink R, Patterson MS, Diamond K, Silver S, Padgett N. Noninvasive measurement of fluorophore concentration in turbid media with a simple fluorescence/reflectance ratio technique. Appl Opt. 2001;40(34):6389-6395.

105. Tian J, Varga B, Somfai GM, Lee W, Smiddy WE, DeBuc DC. Real-time automatic segmentation of optical coherence tomography volume data of the macular region. PloS one. 2015;10(8): $\mathrm{e} 0133908$.

106. Sparrow JR, Dowling JE, Bok D. Understanding RPE lipofuscin. Invest Ophthalmol Vis Sci. 2013;54(13):8325-8326.

107. Ly A, Nivison-Smith L, Assaad N, Kalloniatis M. Fundus autofluorescence in agerelated macular degeneration. Optom Vis Sci. 2017;94(2):246-259. 
108. Falfoul Y, Habibi I, Turki A, et al. Phenotypic progression of stargardt disease in a large consanguineous tunisian family harboring new ABCA4 mutations. Journal of ophthalmology. 2018;2018.

109. Duncker T, Marsiglia M, Lee W, et al. Correlations among near-infrared and shortwavelength autofluorescence and spectral-domain optical coherence tomography in recessive stargardt disease. Invest Ophthalmol Vis Sci. 2014;55(12):8134-8143.

110. Coussa RG, Utz VM, Traboulsi EI. Stargardt disease (STGD). .

111. Sakai N, Decatur J, Nakanishi K, Eldred GE. Ocular age pigment “A2-E”: An unprecedented pyridinium bisretinoid. J Am Chem Soc. 1996;118(6):1559-1560.

112. Lamb LE, Simon JD. A2E: A component of ocular lipofuscin. Photochem Photobiol. 2004;79(2):127-136.

113. Eldred GE. Lipofuscin fluorophore inhibits lysosomal protein degradation and may cause early stages of macular degeneration. Gerontology. 1995;41:15.

114. Mihai DM, Washington I. Vitamin A dimers trigger the protracted death of retinal pigment epithelium cells. Cell death \& disease. 2014;5(7):e1348.

115. Maeda A, Maeda T, Golczak M, Palczewski K. Retinopathy in mice induced by disrupted all-trans-retinal clearance. J Biol Chem. 2008;283(39):26684-26693.

116. Organisciak DT, Winkler BS. Retinal light damage: Practical and theoretical considerations. Prog Retin Eye Res. 1994;13(1):1-29. 
117. Wielgus AR, Lih FB, Tomer KB, Chignell CF, Roberts JE. Blue light induces A2E oxidation in rat eyes. Invest Ophthalmol Vis Sci. 2006;47(13):1533.

118. Ren RX, Sakai N, Nakanishi K. Total synthesis of the ocular age pigment A2-E: A convergent pathway. J Am Chem Soc. 1997;119(15):3619-3620.

119. Rivett E. Synthesis and polarographic analysis of A2E. . 2013.

120. Penn J, Mihai DM, Washington I. Morphological and physiological retinal degeneration induced by intravenous delivery of vitamin A dimers in rabbits. Dis Model Mech. 2015;8(2):131-138.

121. Kotnala A, Senthilkumari S, Halder N, Kumar A, Velpandian T. Microwave assisted synthesis for A2E and development of LC-ESI-MS method for quantification of ocular bisretinoids in human retina. Journal of Chromatography B. 2018;1073:10-18.

122. Parish CA, Hashimoto M, Nakanishi K, Dillon J, Sparrow J. Isolation and one-step preparation of $\mathrm{A} 2 \mathrm{E}$ and iso-A2E, fluorophores from human retinal pigment epithelium. Proc Natl Acad Sci U S A. 1998;95(25):14609-14613.

123. Radu RA, Mata NL, Bagla A, Travis GH. Light exposure stimulates formation of A2E oxiranes in a mouse model of stargardt's macular degeneration. Proc Natl Acad Sci U S A. 2004;101(16):5928-5933. 
124. Sparrow JR, Parish CA, Hashimoto M, Nakanishi K. A2E, a lipofuscin fluorophore, in human retinal pigmented epithelial cells in culture. Invest Ophthalmol Vis Sci. 1999;40(12):2988-2995.

125. White E, Harper W. Synthesis and cyclic voltammetry of A2E. . 2010.

126. Marsiglia M, Lee W, Mahajan VB, et al. Quantitative autofluorescence as a clinical tool for expedited differential diagnosis of retinal degeneration. JAMA ophthalmology. 2015;133(2):219-220.

127. Mitchell GR. Structure of polymer glasses: Short-range order. . 2001.

128. Samavedi S, Poindexter LK, Van Dyke M, Goldstein AS. Synthetic biomaterials for regenerative medicine applications. In: Regenerative medicine applications in organ transplantation. Elsevier; 2014:81-99.

129. Corp M. NANO PMMA and copolymer. . 2001;2019.

130. Prat D, Hayler J, Wells A. A survey of solvent selection guides. Green Chem. 2014;16(10):4546-4551.

131. Construction of Spectro-Fluorometer to acquire, florescence spectrum. Project for master's in biomedical engineering. .

132. Jin Q, Dong X, Chen J, Yao K, Wu Y. Effects of organic solvents on two retinal pigment epithelial lipofuscin fluorophores, A2E and all-trans-retinal dimer. Journal of Zhejiang University-SCIENCE B. 2014;15(7):661-669. 
133. Rurack K. Fluorescence quantum yields: Methods of determination and standards. In: Standardization and quality assurance in fluorescence measurements I. Springer; 2008:101-145.

134. Prahl S. Rhodamine B. https://omlc.org/spectra/PhotochemCAD/html/009.html. Updated 2017.

135. Prahl S. Fluorescein . https://omlc.org/spectra/PhotochemCAD/html/037.html. Updated 2017.

136. Ben-Shabat S, Parish CA, Vollmer HR, et al. Biosynthetic studies of A2E, a major fluorophore of retinal pigment epithelial lipofuscin. J Biol Chem. 2002;277(9):71837190.

137. Brouwer AM. Standards for photoluminescence quantum yield measurements in solution (IUPAC technical report). Pure and Applied Chemistry. 2011;83(12):2213-2228.

138. Gaillard ER, Atherton SJ, Eldred G, Dillon J. Photophysical studies on human retinal lipofuscin. Photochem Photobiol. 1995;61(5):448-453.

139. Ragauskaite L, Heckathorn RC, Gaillard ER. Environmental effects on the photochemistry of A2-E, a component of human retinal lipofuscin. Photochem Photobiol. 2001;74(3):483-488.

140. Baxi J, Calhoun WR, Sepah YJ, et al. Retina-simulating phantom for optical coherence tomography. J Biomed Opt. 2013;19(2):021106. 
141. Lemaillet P, Ramella-Roman JC. Dynamic eye phantom for retinal oximetry measurements. J Biomed Opt. 2009;14(6):064008.

142. Hammer M, Roggan A, Schweitzer D, Muller G. Optical properties of ocular fundus tissues-an in vitro study using the double-integrating-sphere technique and inverse monte carlo simulation. Physics in Medicine \& Biology. 1995;40(6):963.

143. Baxi J, Calhoun W, Sepah YJ, et al. Retina-simulating phantom for optical coherence tomography. J Biomed Opt. 2014;19(2):021106.

144. Hammer M, Schweitzer D. Quantitative reflection spectroscopy at the human ocular fundus. Physics in Medicine \& Biology. 2002;47(2):179.

145. Pollreisz A, Messinger JD, Sloan KR, et al. Visualizing melanosomes, lipofuscin, and melanolipofuscin in human retinal pigment epithelium using serial block face scanning electron microscopy. Exp Eye Res. 2018;166:131-139.

146. Boulton ME. Studying melanin and lipofuscin in RPE cell culture models. Exp Eye Res. 2014;126:61-67.

147. Seagle BL, Rezai KA, Kobori Y, Gasyna EM, Rezaei KA, Norris JR,Jr. Melanin photoprotection in the human retinal pigment epithelium and its correlation with lightinduced cell apoptosis. Proc Natl Acad Sci U S A. 2005;102(25):8978-8983. 
148. Mondragón R, Frixione E. Retinomotor movements in the frog retinal pigment epithelium: Dependence of pigment migration on na and Ca2. Exp Eye Res. 1989;48(5):589-603.

149. Zhang Q, Lu R, Messinger JD, Curcio CA, Guarcello V, Yao X. In vivo optical coherence tomography of light-driven melanosome translocation in retinal pigment epithelium. Scientific reports. 2013;3:2644.

150. Burnside B, Adler R, O'connor P. Retinomotor pigment migration in the teleost retinal pigment epithelium. I. roles for actin and microtubules in pigment granule transport and cone movement. Invest Ophthalmol Vis Sci. 1983;24(1):1-15.

151. Lythgoe JN, Shand J. Endogenous circadian retinomotor movements in the neon tetra (paracheirodon innesi). Invest Ophthalmol Vis Sci. 1983;24(9):1203-1210.

152. Meleppat RK, Zhang P, Ju MJ, et al. Directional optical coherence tomography reveals melanin concentration-dependent scattering properties of retinal pigment epithelium. J Biomed Opt. 2019;24(6):066011.

153. R Sparrrow J, Hicks D, P Hamel C. The retinal pigment epithelium in health and disease. Curr Mol Med. 2010;10(9):802-823.

154. Chong SP, Zhang T, Kho A, Bernucci MT, Dubra A, Srinivasan VJ. Ultrahigh resolution retinal imaging by visible light OCT with longitudinal achromatization. Biomedical optics express. 2018;9(4):1477-1491. 
155. Baumann B, Baumann SO, Konegger T, et al. Polarization sensitive optical coherence tomography of melanin provides intrinsic contrast based on depolarization. Biomedical optics express. 2012;3(7):1670-1683.

156. Wilk MA, Huckenpahler AL, Collery RF, Link BA, Carroll J. The effect of retinal melanin on optical coherence tomography images. Translational vision science \& technology. 2017;6(2):8.

157. Sparrow JR, Wu Y, Nagasaki T, Yoon KD, Yamamoto K, Zhou J. Fundus autofluorescence and the bisretinoids of retina. Photochemical \& Photobiological Sciences. 2010;9(11):1480-1489.

158. Bashkatov AN, Genina EA, Kochubey VI, et al. Optical properties of melanin in the skin and skinlike phantoms. . 2000;4162:219-226.

159. Pogue BW, Patterson MS. Review of tissue simulating phantoms for optical spectroscopy, imaging and dosimetry. J Biomed Opt. 2006;11(4):041102.

160. Hermanson GT. Chapter 10- fluorescent probes. In: Bioconjugate techniques. Academic press; 2013.

161. Curcio CA, Zanzottera EC, Ach T, Balaratnasingam C, Freund KB. Activated retinal pigment epithelium, an optical coherence tomography biomarker for progression in agerelated macular degeneration. Invest Ophthalmol Vis Sci. 2017;58(6):BIO211-BIO226. 
162. Olaf Strauss. PART II: ANATOMY AND PHYSIOLOGY OF THE RETINA. In: eds H. Kolb, R 341 Nelson, E. Fernandez, \& B. Jones, ed. The organization of the retina and visual system. Vol 2019. WEBVISION The Organization of the Retina and Visual System; 2012. https://webvision.med.utah.edu/book/part-ii-anatomy-andphysiology-of-the-retina/the-retinal-pigment-epithelium/.

163. Young RW. The renewal of photoreceptor cell outer segments. J Cell Biol. 1967;33(1):61-72.

164. Young RW, Bok D. Participation of the retinal pigment epithelium in the rod outer segment renewal process. J Cell Biol. 1969;42(2):392-403.

165. Moreno-García A, Kun A, Calero O, Medina M, Calero M. An overview of the role of lipofuscin in age-related neurodegeneration. Frontiers in neuroscience. 2018;12:464.

166. Sparrow JR. Lipofuscin of the retinal pigment epithelium. Atlas of fundus autofluorescence imaging.Springer, Heidelberg. 2007:1-16.

167. Petrukhin K. Pharmacological inhibition of lipofuscin accumulation in the retina as a therapeutic strategy for dry AMD treatment. Drug Discovery Today: Therapeutic Strategies. 2013;10(1):e11-e20.

168. Ma L, Kaufman Y, Zhang J, Washington I. C20-D3-vitamin A slows lipofuscin accumulation and electrophysiological retinal degeneration in a mouse model of stargardt disease. J Biol Chem. 2011;286(10):7966-7974. 
169. Schmitz-Valckenberg S, Holz FG, Bird AC, Spaide RF. Fundus autofluorescence imaging: Review and perspectives. Retina. 2008;28(3):385-409.

170. Schuerch K, Woods RL, Lee W, et al. Quantifying fundus autofluorescence in patients with retinitis pigmentosa. Invest Ophthalmol Vis Sci. 2017;58(3):1843-1855.

171. Sparrow JR, Duncker T, Woods R, Delori FC. Quantitative fundus autofluorescence in best vitelliform macular dystrophy: RPE lipofuscin is not increased in non-lesion areas of retina. In: Retinal degenerative diseases. Springer; 2016:285-290.

172. Delori FC, Keilhauer C, Sparrow JR, et al. Origin of fundus autofluorescence. Atlas of Fundus Autofluorescence Imaging.Berlin Heidelberg: Springer-Verlag. 2007:17-29.

173. Classics Bligh EG, Dyer WJ. A rapid method of total lipid extraction and purification. Can.J.Biochem.Physiol. 1959;37:911-917.

174. Wen R, Lam BL, Guan Z. Aberrant dolichol chain lengths as biomarkers for retinitis pigmentosa caused by impaired dolichol biosynthesis. J Lipid Res. 2013;54(12):35163522.

175. Harkewicz R, Du H, Tong Z, et al. Essential role of ELOVL4 protein in very long chain fatty acid synthesis and retinal function. J Biol Chem. 2012;287(14):11469-11480.

176. Estandarte AK, Botchway S, Lynch C, Yusuf M, Robinson I. The use of DAPI fluorescence lifetime imaging for investigating chromatin condensation in human chromosomes. Scientific reports. 2016;6:31417. 
177. Kapuscinski J. DAPI: A DNA-specific fluorescent probe. Biotechnic \& Histochemistry. 1995;70(5):220-233.

178. Blaszczyk WM, Arning L, Hoffmann K, Epplen JT. A tyrosinase missense mutation causes albinism in the wistar rat. Pigment cell research. 2005;18(2):144-145.

179. Kuramoto T, Nakanishi S, Ochiai M, Nakagama H, Voigt B, Serikawa T. Origins of albino and hooded rats: Implications from molecular genetic analysis across modern laboratory rat strains. PloS one. 2012;7(8):e43059.

180. Sarna T, Burke JM, Korytowski W, et al. Loss of melanin from human RPE with aging: Possible role of melanin photooxidation. Exp Eye Res. 2003;76(1):89-98.

181. Yi J, Chen S, Shu X, Fawzi AA, Zhang HF. Human retinal imaging using visiblelight optical coherence tomography guided by scanning laser ophthalmoscopy. Biomedical optics express. 2015;6(10):3701-3713.

182. Chong SP, Bernucci M, Radhakrishnan H, Srinivasan VJ. Structural and functional human retinal imaging with a fiber-based visible light OCT ophthalmoscope. Biomedical optics express. 2017;8(1):323-337.

183. Shu X, Beckmann L, Wang Y, et al. Designing visible-light optical coherence tomography towards clinics. Quantitative Imaging in Medicine and Surgery. 2019;9(5):769-781. 
184. Zhang T, Kho AM, Srinivasan VJ. Improving visible light OCT of the human retina with rapid spectral shaping and axial tracking. Biomedical Optics Express. 2019;10(6):2918-2931.

185. Delori FC, Webb RH, Sliney DH. Maximum permissible exposures for ocular safety (ANSI 2000), with emphasis on ophthalmic devices. JOSA A. 2007;24(5):1250-1265.

186. Nafar Z, Wen R, Guan Z, Li Y, Jiao S. Quantifying lipofuscin in retinal pigment epithelium in vivo by visible-light optical coherence tomography-based multimodal imaging. Scientific Reports. 2020;10(1):1-10. This work is licensed under the Creative Commons Attribution 4.0 International License. To view a copy of this license, visit http://creativecommons.org/licenses/by/4.0/ or send a letter to Creative Commons, PO Box 1866, Mountain View, CA 94042, USA.

187. ANSI. American national standard for safe use of lasers ANSI Z136. 1-2014. . 2014.

188. Delori FC, Goger DG, Dorey CK. Age-related accumulation and spatial distribution of lipofuscin in RPE of normal subjects. Invest Ophthalmol Vis Sci. 2001;42(8):18551866.

189. Chong SP, Merkle CW, Leahy C, Radhakrishnan H, Srinivasan VJ. Quantitative microvascular hemoglobin mapping using visible light spectroscopic optical coherence tomography. Biomedical optics express. 2015;6(4):1429-1450. 
190. Baghaie A, Yu Z, D'Souza RM. Involuntary eye motion correction in retinal optical coherence tomography: Hardware or software solution? Med Image Anal. 2017;37:129145. 


\section{APPENDICES}

\section{FLORIDA
INTERNATIONAL
UNIVERSITY}

\section{MEMORANDUM}

To:

Dr. Shuliang Jiao

$\mathrm{CC}$ :

File

From:

Maria Melendez-Vargas, MIBA, IRB Coordinator

Date:

April 4, 2019

Protocol Title: "Multimodal Eye Imaging"
Office of Research Integrity Research Compliance, MARC 414

The Health Sciences Institutional Review Board of Florida International University has approved your study for the use of human subjects via the Expedited Review process. Your study was found to be in compliance with this institution's Federal Wide Assurance (00000060).
IRB Protocol Approval \#: IRB-19-0112
IRB Approval Date: $\quad 04 / 04 / 19$
TOPAZ Reference \#:
107682
IRB Expiration Date: $\quad 04 / 04 / 22$

As a requirement of IRB Approval you are required to:

1) Submit an IRB Amendment Form for all proposed additions or changes in the procedures involving human subjects. All additions and changes must be reviewed and approved by the IRB prior to implementation.

2) Promptly submit an IRB Event Report Form for every serious or unusual or unanticipated adverse event, problems with the rights or welfare of the human subjects, and/or deviations from the approved protocol.

3) Utilize copies of the date stamped consent document(s) for obtaining consent from subjects (unless waived by the IRB). Signed consent documents must be retained for at least three years after the completion of the study.

4) Obtain continuing review and re-approval of the study prior to the IRB expiration date. Submit the IRB Renewal Form at least 30 days in advance of the study's expiration date.

5) Submit an IRB Project Completion Report Form when the study is finished or discontinued.

HIPAA Privacy Rule: N/A

Special Conditions: N/A

For further information, you may visit the IRB website at http://research.fiu.edu/irb.

$\mathrm{MMV} / \mathrm{em}$ 


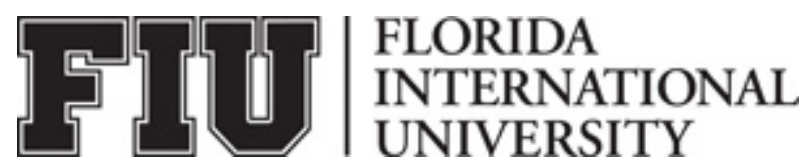

\section{ADULT CONSENT TO PARTICIPATE IN A RESEARCH STUDY}

\author{
Multimodal Retinal Imaging
}

\section{SUMMARY INFORMATION}

Things you should know about this study:

- Purpose: The purpose of this study is to test and validate a retinal imaging technology.

- Procedures: If you choose to participate, you will be asked to sit in front of the imaging system and keep your eyes steady during the acquisition (1-4 seconds). Ones in dark and another time in a bright room light.

- Duration: This will take about 60 minutes.

- Risks: The main risk or discomfort from this research is discomfort from bright light illumination for 4 seconds.

- Benefits: There is no direct benefit to you. However, development of this imaging technology will in the future benefit patients with retinal degenerative diseases.

- Alternatives: There are no known alternatives available to you other than not taking part in this study.

- Participation: Taking part in this research project is voluntary.

Please carefully read the entire document before agreeing to participate.

\section{PURPOSE OF THE STUDY}

The purpose of this study is to test and validate a retinal imaging technology. The ultimate goal of the technology is to provide diagnosis and monitoring of retinal degenerative disease like Age related Macular Degeneration (AMD). 


\section{NUMBER OF STUDY PARTICIPANTS}

If you decide to be in this study, you will be one of 30 people in this research study.

\section{DURATION OF THE STUDY}

Your participation will involve 60 minutes.

\section{PROCEDURES}

If you agree to be in the study, we will ask you to do the following things:

1. You may be asked to stay in a darkroom with dim red light for about 30 minutes. This is a process called dark adaptation.

2. You will be asked to sit in front of the imaging system and put your chin on the chinrest.

3. You will be asked to look at a red fixation light while the operator is aligning the system. During the alignment process you will see a red cross, which is the scanning light in the near infrared for OCT imaging.

4. When the alignment is good you will be asked to open your eye wide and keep your eye steady. Then a blue light will shine into and scan across your eye for about 1 to 4 seconds. You should keep your eye steady and do not follow the scanning light.

5. The room light will be turned on and your eyes will adapt to the bright room light.

6. We will take another image of the same eye with the above procedure.

\section{RISKS AND/OR DISCOMFORTS}

There is no risk associated with this study. However, you may feel some discomfort about bright light illumination during the image acquisition time (1-4 seconds).

\section{BENEFITS}

The study has the following possible benefits to you/society: Your participation will help us validate and refine the imaging technology. Development of this imaging technology will in the future benefit patients with retinal degenerative diseases by offering early diagnosis and provide them better opportunity to get treatment before losing their vision when therapy is available. Further, this device will help physicians to monitor disease 
progression and treatment by providing a standardized measurement of fundus autofluorescence.

\section{ALTERNATIVES}

There are no known alternatives available to you other than not taking part in this study. Any significant new findings developed during the course of the research which may relate to your willingness to continue participation will be provided to you.

\section{CONFIDENTIALITY}

The records of this study will be kept private and will be protected to the fullest extent provided by law. In any sort of report, we might publish, we will not include any information that will make it possible to identify you. Research records will be stored securely, and only the researcher team will have access to the records. However, your records may be inspected by authorized University or other agents who will also keep the information confidential.

- To help us protect your privacy, we have a Certificate of Confidentiality from the National Institutes of Health (NIH). With this Certificate, we can't be forced by a court order or subpoena to disclose information that could identify you in any civil, criminal, administrative, legislative or other proceedings.

- There are circumstances where the Certificate doesn't protect against disclosure of your personally identifiable information:

- when the US government is inspecting or evaluating federally-funded studies

- when information must be disclosed to meet FDA requirements

- if you give someone written permission to receive research information or you voluntarily disclose your study information

- if the researcher reports that you threatened to harm yourself or others

- in cases of child abuse reported by the researcher

- if the investigator reports cases of contagious disease (such as HIV) to the state

\section{USE OF YOUR INFORMATION}


Identifiers about you might be removed from the identifiable private information and that, after such removal, the information could be used for future research studies or distributed to another investigator for future research studies without additional informed consent from you or your legally authorized representative

\section{COMPENSATION \& COSTS}

There is no compensation provided to you and you will not be responsible for any costs to participate in this study.

\section{RIGHT TO DECLINE OR WITHDRAW}

Your participation in this study is voluntary. You are free to participate in the study or withdraw your consent at any time during the study. You will not lose any benefits if you decide not to participate or if you quit the study early. The investigator reserves the right to remove you without your consent at such time that he/she feels it is in the best interest.

\section{RESEARCHER CONTACT INFORMATION}

If you have any questions about the purpose, procedures, or any other issues relating to this research study you may contact Zahra Nafar at FIU MMC OE 132, (305)348-5897, znafar@fiu.edu.

\section{IRB CONTACT INFORMATION}

If you would like to talk with someone about your rights of being a subject in this research study or about ethical issues with this research study, you may contact the FIU Office of Research Integrity by phone at 305-348-2494 or by email at ori@fiu.edu. 


\section{PARTICIPANT AGREEMENT}

I have read the information in this consent form and agree to participate in this study. I have had a chance to ask any questions I have about this study, and they have been answered for me. I understand that I will be given a copy of this form for my records.

Signature of Participant Date

Printed Name of Participant

Signature of Person Obtaining Consent Date 
VITA

Zahra Nafar

Born, Tehran, Iran

2008-20013 B.Sc., Electrical Engineering, Bioelectrics

University of Tehran

Tehran, Iran

2014-2020 M.S. and PhD, Biomedical Engineering

Florida International university

Miami, Florida

PUBLICATIONS

+ Zahra Nafar, Rong Wen, Ziqiang Guan, Yiwen Li, and Shuliang Jiao, "Quantifying lipofuscin in retinal pigment epithelium in vivo by visible-light optical coherence tomography-based multimodal imaging", Scientific Reports (2020)

+ Zahra Nafar, Rong Wen, Shuliang Jiao, "Visible-light optical coherence tomographybased multimodal system for quantitative fundus autofluorescence imaging", Experimental Biology and Medicine (2018)

+ Z. Nafar, R. Wen, and Sh. Jiao," Visible light OCT-based quantitative imaging of lipofuscin in the retinal pigment epithelium with standard reference targets", Biomed. Opt. Express (2018)

+ Z. Nafar, M. Jiang, R. Wen, and Sh. Jiao, "Visible-light optical coherence tomographybased multimodal retinal imaging for improvement of fluorescent intensity quantification", Biomed. Opt. Express (2016)

+ A. Zaeemzadeh, Z. Nafar, K. Setarehdan, " Heart sound segmentation based on recurrence time statistics", 20th Iranian Conference of Biomedical Engineering (ICBME), IEEE, (2013) 


\section{PRESENTATIONS}

+ Zahra Nafar, Rong Wen, Shuliang Jiao, "In vivo quantification of RPE lipofuscin with visible light OCT-based fundus autofluorescent and A2E reference target", SPIE conference (2020)

+ Zahra Nafar, Rong Wen, Shuliang Jiao, "VIS-OCT based quantitative imaging of RPE Lipofuscin with customized A2E-PMMA reference target", SPIE conference (2019)

+ Zahra Nafar, Rong Wen, Shuliang Jiao, "Visible Light OCT-based Quantitative imaging of Lipofuscin in the RPE with Standard Reference Targets", ARVO meeting (2018)

+ Zahra Nafar, Shuliang Jiao, "Visible Light Optical Coherence Tomography based Quantification of Lipofuscin together with a Standard Reference Target", SPIE conference (2018)

+ Zahra Nafar, Tan Liu, Rong Wen, Byron L. Lam, Carmen A. Puliafito \& Shuliang Jiao," Rhodopsin molecular contrast imaging by optical coherence tomography for functional assessment of photoreceptors", SPIE conference (2016) 